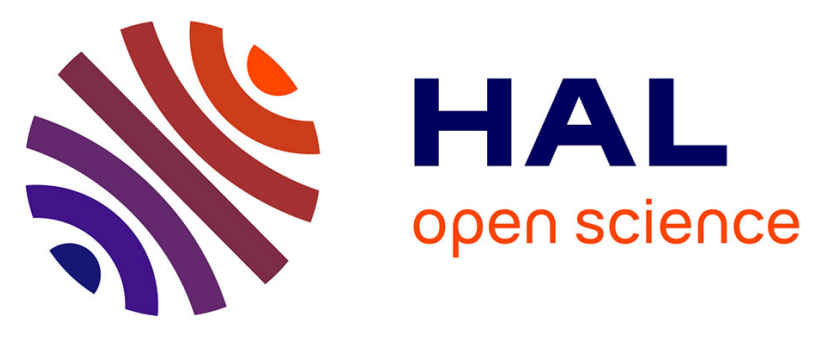

\title{
Progress regarding the context-of-use of tau as biomarker of Alzheimer's disease and other neurodegenerative diseases
}

Nicole Campese, Giovanni Palermo, Claudia Del Gamba, Maria Francesca Beatino, Alessandro Galgani, Elisabetta Belli, Eleonora Del Prete, Alessandra Della Vecchia, Andrea Vergallo, Gabriele Siciliano, et al.

\section{To cite this version:}

Nicole Campese, Giovanni Palermo, Claudia Del Gamba, Maria Francesca Beatino, Alessandro Galgani, et al.. Progress regarding the context-of-use of tau as biomarker of Alzheimer's disease and other neurodegenerative diseases. Expert Review of Proteomics, 2021, 10.1080/14789450.2021.1886929. hal-03138524

\section{HAL Id: hal-03138524 \\ https://hal.sorbonne-universite.fr/hal-03138524}

Submitted on 11 Feb 2021

HAL is a multi-disciplinary open access archive for the deposit and dissemination of scientific research documents, whether they are published or not. The documents may come from teaching and research institutions in France or abroad, or from public or private research centers.
L'archive ouverte pluridisciplinaire HAL, est destinée au dépôt et à la diffusion de documents scientifiques de niveau recherche, publiés ou non, émanant des établissements d'enseignement et de recherche français ou étrangers, des laboratoires publics ou privés. 


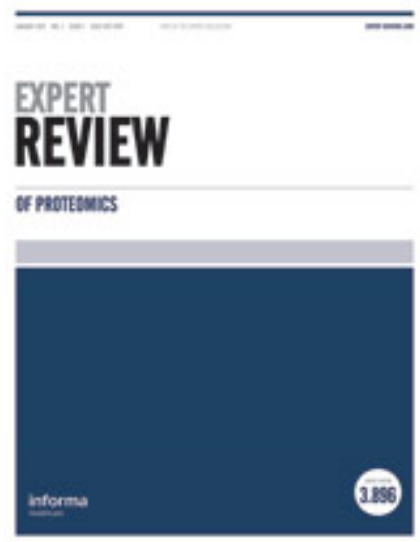

\section{Progress regarding the context-of-use of tau as biomarker of Alzheimer's disease and other neurodegenerative diseases}

\begin{tabular}{|r|l|}
\hline Journal: & Expert Review of Proteomics \\
\hline Manuscript ID & ERU-2020--0079.R1 \\
\hline Manuscript Type: & Review (Invited) \\
\hline Keywords: & $\begin{array}{l}\text { Alzheimer's Disease, Fluid biomarkers, Neurodegenerative diseases, Tau, } \\
\text { Tau-PET, Ultrasensitive techniques }\end{array}$ \\
\hline
\end{tabular}

\section{SCHOLARONE \\ Manuscripts}


Progress regarding the context-of-use of tau as biomarker of Alzheimer's disease and other neurodegenerative diseases

\begin{abstract}
Introduction Tau protein misfolding and accumulation in toxic species is a critical pathophysiological process of Alzheimer's Disease (AD) and other neurodegenerative disorders (NDDs). Tau biomarkers, namely cerebrospinal fluid (CSF) total-tau (t-tau), 181-phosphorylated tau (p-tau) and tau--PET tracers, have been recently embedded in the diagnostic criteria for AD. Nevertheless, the role of tau as a diagnostic and prognostic biomarker for other NDDs still remains controversial.

Areas covered We performed a systematical_PubMed-basedly review ofed the most recent advances in tau-related biomarkers for NDDs. We focused on papers published in the last five yearsfrom 2015 to 2020, assessing the diagnostic or prognostic value of each biomarker.

Expert opinion The assessment of tau biomarkers in alternative easily accessible matrices, through the development of ultrasensitive techniques, represents the most significant perspective for ADbiomarker research. In NDDs, novel tau isoforms (eg.e.g., 217p-taup-tau217) or proteolytic fragments (e.g.e.g., N-terminal fragments) may represent candidate diagnostic and prognostic biomarkers and may help monitoring disease progression. Protein misfolding amplification assays, allowing the identification of different tau strains (e.g. 3R- vs. 4R-tau) in CSF, may constitute a breakthrough for the in vivo stratification of NDDs. Tau-PET may help tracking the spatialtemporal evolution of tau pathophysiology in $\mathrm{AD}$ but its application outside the $\mathrm{AD}$-spectrum deserves further studies.:-
\end{abstract}

Keywords: Alzheimer's Disease, Fluid biomarkers, Neurodegenerative diseases, Tau, Tau-PET, Ultrasensitive techniques 


\section{Article highlights}

- Tau protein misfolding and accumulation in toxic species and tangles is a critical pathophysiological process of Alzheimer's Disease (AD) and other neurodegenerative disorders (NDDs);

- Cerebrospinal fluid (CSF) total tau (t-tau) and phosphorylated tau (p-tau) are included in the current diagnostic criteria for AD; in primary tauopathies and in other NDDs (ALS-FTD spectrum and synucleinopathies $\alpha$-synucleinopathies) the role of tau as a diagnostic and prognostic biomarker is still controversial;

- The quantification of t-tau and p-tau and of emerging tau biomarkers in plasma by using ultrasensitive techniques are gaining momentum in the AD diagnostic workup;

- Novel tau fragments and tau isoforms detectable by means of ultrasensitive methods including Protein Misfolding Cyclic Amplification (PMCA) and Real-time quaking-induced conversion (RT-QuIC) may improve the in vivo stratification of NDDs;

- Tau-PET tracers are useful tools for the in vivo tracking of the spatial-temporal evolution of tau pathophysiology in AD but further studies are needed to define their proper context-ofuse in other tauopathies. 


\section{Introduction}

Tau is a natively unfolded microtubule-associated protein largely expressed in the central nervous system[1]. This protein plays an important role in microtubule polymerization, stabilization and remodeling, and is mainly expressed in the axons, where it also participates in regulating cellular transport processes[2,3]. Tau exists in 6 isoforms generated through the alternative splicing of the exons 2, 3 and 10 of the microtubule-associated protein tau (MAPT) gene[4]. The microtubulebinding domain (MBD) is encoded by the exon 10, whose alternative splicing produces 3 isoforms, each with 3 or 4 repeats, respectively called 3-repeat (3R) and 4-repeat (4R) tau[2]. Under pathological conditions, mutations in the MAPT gene along with a hyper-phosphorylation of tau may reduce the affinity of the MBD to the microtubules[5]. Accordingly, tau free-protein concentrations increase to a tipping point after which misfolded protein intermediates self-assembly into unwanted oligomers, fibrils, and neurofibrillary tangles (NFT)[5].

The neuronal and/or glial accumulation of aberrant tau aggregates represents the pathological hallmark of a broad spectrum of neurodegenerative disorders (NDDs) called tauopathies [6]. These include the so-called primary tauopathies, a group of $\mathrm{NDDs}_{2}$ in which tau aggregates, including NFT, represents the main pathological hallmark that drives the neurodegenerative process. Primary tauopathies can be classified into 3R_-tauopathies, 4Rtauopathies and; mixed 3R/4R tauopathies. The major clinical phenotypes belong to the spectrum of Progressive Supranuclear Palsy (PSP), Corticobasal Degeneration (CBD) and Frontotemporal Lobar Degeneration with tau deposits (FTLD-Tau) Frontotemporal-lobar Degeneration associated with MAPT mutations (FTLDMAPT) [2,6]. Secondary tauopathies are conditions resulting by from a complex interplay between tau and other misfolded proteins (e.g., amyloid- $\beta$ - $-(A \beta))$ or related to alternative causes (e.g., autoimmune processes). Alzheimer's Disease (AD) represents the best characterized and more intensively studied NDDs and is classified as secondary tauopathy[2,7]. More recently, other forms of secondary tauopathies have been described including, chronic traumatic encephalopathy (CTE) and the autoimmune, anti-IgLON5 encephalitis [2]. Tau cytoplasmic inclusions have also been reported in the Amyotrophic lateral seleresis Frontotemporal Dementia (FTD) (ALSFTD) spectrum; up to 30\% of patients with the behavioral variant of frontotemporal dementia (bvFTD) and the majority (approximately 90\%) of patients with the non-fluent variant of Primary progressive aphasia (nfvPPA) show tau pathologic depositions [8]. Also in $\alpha$-synucleinopathies, where aberrant $\alpha$-synuclein aggregates underpin the neurodegenerative mechanisms, NFT are likely to cause neurodegeneration and contribute to the disease burden $[9,10]$. 
Tau bBiomarkers tracking tau pathology are included in the diagnostic workup of AD according to the 2011 National Institute on Aging - Alzheimer's Association (NIA-AA), the 2014 International Working Group-2 (IWG-2) criteria and to-the AT(N) classification system [11,12]. In 2018 the $\operatorname{AT}(\mathrm{N})$ scheme embed biomarkers of amyloid- $\beta$-related pathology (A), tau-pathology (T) and other markers of neurodegeneration $(\mathrm{N})$ [13]. This classification includes validated biomarkers to track tau pathology in AD such as cerebrospinal fluid (CSF) total-tau (t-tau), and 181phesphorylated tau (p tau), all increased in $A D$ individuals, as biomarkers of neurodegeneration $(N)$ and 181-phosphorylated-tau (p-tau181 or simply p-tau), as biomarker of tau pathology [13]. These biomarkers are all increased in AD individuals. The AT(N) categorization for the first time added cerebral tau- positron emission tomography (PET) as core biomarker of AD. Indeed, the implementation of tau-PET tracers represents a recent step forward in the AD diagnostic workup [13].

Currently, the ante-mortem diagnosis of non-AD NDDs mainly relies on pure clinically based criteria. In this scenario, the diagnostic and prognostic value of tau protein in the spectrum of primary tauopathies remains unsatisfactory when measured with traditional approaches (e.g. ELISA) [14]. In fact, a biomarkers-based diagnosis is still an unmet need in NDDs and several methodological challenges should be addressed to overcome this limitation. In the last years, the use of ultrasensitive assays, the discovery of alternative tau byproducts in biofluids, and the development of innovative techniques, enabling the differentiation of tau strains, have represented potential breakthroughs for the in vivo dissection of tau pathology spectrum.

The validation of biomarkers tracking neurodegenerative processes and predicting pathophysiological progression at the earliest molecular/cellular changes is an unmet need for the early diagnosis, the prognostic evaluation and -for patients selection and follow-up in targetedtherapyies clinical trials the monitoring of targeted-therapies in clinical trials [15].

We aim to review the current state of the art on the use of tau protein as a biomarker for NDDs. First, we investigated recent advances of this fluid biomarker in the diagnostic and prognostic workup of AD and other NDDs. Secondly, we evaluated the contribution of cerebral PET-imaging in tracking tau pathology across the NDDs spectrum.

\section{Literature search methods}

The aim of the present work is to review the most recent advances on tau as a biomarker for neurodegenerative diseases. A systematic PubMed-based literature search was performed to select relevant papers assessing the diagnostic and/or prognostic value of plasma tau (both t-tau, p-tau, and other tau fragments) in $\mathrm{AD}$ and other NDDs, as evaluated through novel ultrasensitive 
techniques and of CSF tau in other neurodegenerative diseases. We screened the papers using the following combination of keywords:

- for plasma tau in AD and other NDDs: (("plasma" OR "blood" OR "serum") AND ("tau") AND ("Alzheimer" OR "Corticobasal degeneration" OR "Progressive Supranuclear Palsy" OR "Amyotrophic Lateral Sclerosis" OR "Frontotemporal Dementia" OR "Parkinson" OR "Multiple System Atrophy" OR "Lewy Body"));

- for CSF tau in NDDs (AD excluded): (("CSF") AND ("tau") AND ("Corticobasal Degeneration" OR "Progressive Supranuclear Palsy" OR "Amyotrophic Lateral Sclerosis" OR "Frontotemporal Dementia" OR "Parkinson's Disease" OR "Multiple System Atrophy" Or "Dementia With Lewy Bodies")).

Only papers written in English, assessing the prognostic and/or diagnostic value of tau and published between 2015 and 2020, were included in the final analysis. A total number of 1480 and 297 articles for plasma tau and CSF tau respectively were initially screened. Fourteen papers on plasma tau and 14 on CSF tau were retained in the final analysis (Fig.2).

For each study, we analyzed the study population, the technique used for biomarkers detection, the diagnostic and/or prognostic performance of the investigated biomarker. Given the high heterogeneity of the papers included, we used the AuROC values as a concise measure of diagnostic accuracy to compare different studies. The diagnostic value of each marker to properly allocate patients to different diagnostic groups was identified when available and classified as follows: 'excellent' (area under ROC curve [AuROC] 0.90-1.00), 'good' (AuROC: 0.80-0.89), 'fair' (AuROC: 0.70-0.79), 'poor' (AuROC: 0.60-0.69), or 'fail' (i.e., no discriminatory capacity) (AuROC: $0.50-0.59$ ) [16]. We used hazard ratios (HR) to compare the prognostic values. Possible comparisons across studies are only qualitative, and not based on statistical analysis.

\section{Fluid biomarkers tracking tau-pathology: from conventional to ultrasensitive techniques}

Fluid biomarkers represent promising tools for the in vivo tracking of early pathophysiological changes in NDDs. Their detection and quantification in different matrices and related technical issues are regarded as hot topics in biomarkers research[14].

Tau quantification in CSF represents the first and most widely validated approach for the in vivo assessment detection of tau protein pathology. Tau, quantified in the CSF both as total-tau and 181phespherylated $\tan \left(\mathrm{p}\right.$-tau181) currently belongs to the core AD biomarkers, along with amyloid- $\beta_{42}$ $\left(\mathrm{A} \beta_{42}\right)$, and its assessment is based on traditional Enzyme-linked immunoabsorbent assay (ELISA) techniques [11-13]. Accordingly, several commercial kits are to date available for both clinical and research purposes, including the INNOTESTBhTAU $\Lambda \mathrm{g}$ and the INNOTEST PHOSPHO TAU 
[17]. Even if broadly used and largely validated, standard tau quantification techniques present three main limitations: 1) they are unable to effectively quantify tau in "peripheral" matrices (e.g., plasma), where its concentration is significantly low [18], 2) they fail in detecting alternative tau species (e.g., p-p217-tau-217) 3) are ineffective in differentiating tau isoforms (e.g., 3R and 4R tau), seeds (e.g., tau fibrils), based on their tridimensional conformation [19].

Traditional methods show unsatisfactory and suboptimal power in differentiating patients with NDDs from healthy controls (HC) individuals-when applied to biological matrices other than CSF (e.g., blood), where these molecules are expressed at femtomolar ranges instead of picomoles [20]. The quantification of tau in unconventional non-CSF substrates is challenging because the concentration of the analyte may not reflect primary neurodegenerative changes. Unpredictable $\forall$ Variations in tau concentrations could be due to peripheral proteolytic processes, metabolic clearance, the interaction with other proteins and modifications of the of brain blood barrier permeability[21].

Novel techniques, developed and validated in the last five years, show better sensitivity in measuring tau levels than traditional methods by detecting up to subpicomolar and even subfemtomolar concentrations. These techniques mostly address the N-terminal rather than midregion of tau. Single Molecule Array (Simoa), a fully-automated ELISA relying on antibody-coated magnetic beads, has been used to assess t-tau, p-tau [22-24]_and N-terminal tau fragments [25] in blood samples of AD patients (Table 1). On the other hand, Electrochemiluminescence immunoassays (ECLIA) provide a quantification of t-tau [26], p-tau181 [27-29] and p217 tauptau217 [30,31] in patients with AD, with high accuracy even in low sample volumes (Table 1). An upgrade of standard mass spectrometry (MS), combining immunoprecipitation reactions with MS $[32,33]$, tracks different phosphorylated tau isoforms like p217 taup-tau217 and p-tau181 in plasma [33](Table 1). Also immunomagnetic reaction (IMR) - an antibody-mediated reaction generating changes in the magnetic fields that enable the quantification of the analyte [34,35] - detects t-tau [36-39] and p-tau181 [37,39]- concentrations with a greater sensitivity compared to traditional techniques, but with suboptimal specificity (Table 1).

However, these ultrasensitive techniques fail in discriminating different tridimensional conformations of tau (e.g., $3 \mathrm{R}$ vs $4 \mathrm{R}$ tau-seeds). Nevertheless, the selective identification of different tau seeds represents a critical issue to understand the pathophysiology of the primary tauopathiesy spectrum. Based also on the hypothesis of a prion-like transcellular propagation of tau, real-time quaking-induced conversion (RT-QuIC) and protein misfolded cycle amplification (PMCA) have been successfully applied in NDDs [40]. These techniques were initially developed for the detection of prion proteins in CreutzfeldCreutzfeldt-Jakob disease. RT-QuIC uses 


\section{Advances in tau as a biomarker for Alzheimer's Disease: assessing novel matrices, techniques and targets}

T-tau and p-tau proteins measured in CSF are core biomarkers of neurodegeneration in $\mathrm{AD}_{-}[11,12]$. However, their contribution in the identification of non $\triangle D$ taupathies is limited. The finding of alternative tau isoforms specific for the different tauopathies-clinical and pathological AD subtypes may represent a significant advancement, in parallel with the recent development of ultrasensitive techniques that allow the measurement of tau proteins in the-blood.

To overcome several technical issues including the higher dilution of tau biomarkers in blood vs. CSF [42], several antibody-driven assays have been combined with different detection systems reaching subpicomolar and even subfemtomolar limits of detection (LOD) [43]. A metanalysis indicated that plasma t-tau is increased in AD [44]. A study performed with IMR techniques showed that plasma t-tau levels discriminated AD from controls with optimal accuracy (Table 2) [36]. By contrast, other studies conducted with Simoa and IMR reported higher plasma ttau concentrations in $\mathrm{AD}$ or Mild Cognitive Impairment $(\mathrm{MCI})$ patients compared to $\mathrm{HC}$, but with consistent overlapping results $[27,36,39,45,46]$. Furthermore, plasma t-tau is not or only weakly correlated with CSF t-tau, p-tau in different studies [45,47-49]. An inverse association between ttau and cortical thickness in AD-related areas has been reported [38,46]. Plasma t-tau was associated in $\mathrm{AD}, \mathrm{MCI}$ and $\mathrm{HC}$ with a longitudinal decline of cognitive scores and cerebral 18FDGuptake at PET imaging, increased ventricular volume, and a decrease of hippocampal volume [47,50]. Combining plasma t-tau into ratios (e.g., with plasma amyloid- $\beta_{1-42}$ ) increases the predictive value of cerebral tau accumulation, as assessed by PET, compared to t-tau alone [22]. On the other hand, plasma t-tau did not predict the amyloid- $\beta$--status as assessed by cerebral amyloidPET [27] or CSF [26] in AD, MCI, and HC. Intriguingly baseline plasma t-tau levels seem to be associated with atrophy of the basal forebrain cholinergic system (BFCS) in subjective memory 
complainers, regardless of their amyloid- $\beta$-status [51], although no differences in plasma t-tau levels emerged between SCD and HC in a further study [48]. Finally, plasma t-tau levels were found to be associated with MAPT H1c haplotype in $\mathrm{AD}, \mathrm{MCI}$ and $\mathrm{HC}$, according to a genomewide association study (GWAS) [52].

Full-length (FL) tau may account only for a small part of plasma tau proteins. Different proteolytic fragments of tau have been explored in plasma with ultrasensitive techniques so far. The levels of tau N1 fragments, measured with Simoa, are higher in AD and AD-MCI subjects than HC, showing good to optimal accuracy in discriminating HC from AD and MCI subjects, respectively [25] (Table 2). The actual eligibility of these fragments is still a matter of debate [53].

Conversely, pPlasma p-tau181 seems to be a rebust-more specific AD biomarker. Plasma ptau181 concentrations assessed by Simoa, IMR and ECLIA-based techniques are higher in AD and MCI-AD compared to HCs [23,27-29,39]. Plasma p-tau181 distinguishes AD patients from non$\mathrm{AD}$ dementias taken together [28] and from vascular dementia (VaD) with optimal accuracy [23], from FT $₫ D$ with good to optimal accuracy $[23,29]$ and from PSP, CBD and Parkinson's disease $(\mathrm{PD})$ or Multiple System Atrophy (MSA) with good accuracy [23] (Table 2). Furthermore, plasma p-tau concentration is consistent with CSF p-tau levels in A $\beta$-PET positive individuals $[28,29]$. Plasma p-tau181 predicts the A $\beta$-PET status with good accuracy and is associated with both $A \beta$ and tau PET positivity in MCI, AD, and HC [27] (Table 2). Moreover, in a cohort of AD, MCI and HC, plasma p-tau181 correlated with the spreading of taupathy tau deposition within the brain [22].

Tau isoforms other than p-tau181 have been recently studied and showed a potential role in the differential diagnosis of AD from MCI and HC. Plasma p-tau217 was able to differentiate AD (clinically assessed) from non-AD patients and autopsy-confirmed AD from non-AD patients with slightly suboptimal accuracy [30] (Table 2). Plasma p-tau-217 levels predict the amyloid- $\beta$--status as assessed by amyloid-PET or CSF A $\beta 42 / \mathrm{A} \beta 40$ ratio with optimal accuracy across preclinical-AD, AD-MCI, moderate AD, non-AD-MCI and HC [33] (Table 2). Furthermore p-tau217 could discriminate amyloid- $\beta$ positive and tau-PET negative participants from $\mathrm{HC}$ and unreverled itshas a potential role as an early, preclinical biomarker, being able to assess tau increase before detectable tau aggregation. Both plasma p-tau217 and p-tau181 correlated with their CSF counterparts and were shown to be released from CNS rather than from peripheral sources, thus probably representing more reliable $\mathrm{AD}$ biomarkers compared tothan plasma t-tau and p-tau202 [33]. Moreover ${ }_{2}$ the cerebral $\mathrm{A} \beta$ plaques and tau tangles accumulation are is independently associated with ante-mortem concentrations of plasma p-tau217 in an autopsy-confirmed cohort [54]:

Recently, dynamic trajectories of subsequent site-specific phosphorylation of tau have been reported from preclinical to full-blown AD stages in CSF. While p-tau217 and p-tau181 increase as 
early as two decades before and tend to decline close to the symptomatic stage, the levels of t-tau and p-tau205 increase later (between 17-13 estimated years to symptom onset) and constantly increase along disease progression [55] AAlso p-tau231 assessed in CSF by using a mid-region target antibody increases in preclinical AD, largely before p-tau181 and p-tau217, when only subtle $A \beta$ pathology is detectable [56]. These preliminary data suggest the potential role of alternative sitespecific phosphorylated isoforms of CSF p-tau for the identification of preclinical AD and for the subsequent risk of phenoconversion. Dynamic changes of tau phosphorylation profile may also represent a potential useful biomarker to assess the efficacy of disease modifying therapies in clinical trials but a better understanding of these changes and a more extensive validation of these biomarkers and their potential context-of--use is needed. Furthermore, whether these changes are reflected in plasma and their timing is ana questionan issue that still needs toshould be assessed.

Full (FI) proteolytic fragments of tau have been explored in plasma with ultrasensitive techniques so far. The

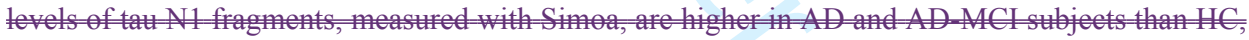
sh [24] (Table 2). The actual eligibility of these fragments is still a matter of debate [52].

\section{The diagnostic and prognostic role of tau as a biomarker in $4 \mathrm{R}$-tauopathies}

Tauopathies are pathologically characterized by a predominant intracellular accumulation of hyperphosphorylated tau fibrils [1,2] Primary tauopathies can be divided into 3R-tauopathies, including Pick's Disease (PiD), and 4R-tauopathies like Progressive Suprantelear Palsy $\left(\mathrm{PSP}_{2}\right.$, Corticobasal Degeneration (CBD), Argyrophilic Grain Disease (AGD), Globular Glial Tauopathy (GGT). Mixed 3R- and 4R-tauopathies include Primary Age-Related Tauopathy (PART) and neurofibrillary tangles (NFT)-dementia[2]. The genetic FTLD due to MAPT mutations may be related either to 3R-, 4R- or 3/4 R-tau pathological patterns [3](Figure 1). The clinical phenotype of these forms is highly heterogeneous and may not reflect the underlying pathology $[14,57]$.

Currently, the diagnosis of 4R-tauopathies relies on the post-mortem detection of diseasespecific patterns of abnormal tau aggregates [3]. Nevertheless, the in vivo diagnosis of these disorders is based on purely clinical-based criteria without reliable biomarkers to track the different tau pathologies $[3,14,58]$.

The potential role of CSF tau concentration as a diagnostic and prognostic biomarker in 4Rtauopathies (especially PSP and corticobasal syndrome - CSBS ) has been investigated by several studies since 1997, with conflicting results. The measurement of CSF t-tau and p-tau concentrations is helpful in the diagnostic workup of AD. Indeed the characteristic increase of tau species (t-tau or

\section{Commented [EB3]: idem}

Commented [EB4]: idem

Commented [EB5]: idem 
p-tau) in the CSF is specific to AD pathology and lacks in non-AD dementias (including PSP and CBS) $[59,60]$. Further, CSF t-tau and p-tau do not distinguish PD from atypical parkinsonism with enough accuracy (in particular from PSP) [61]. Nevertheless these biomarkers when combined with others in CSF - $\alpha$-synuclein, A $\beta 42$, neurofilament (NFL) [62]-_-soluble amyloid precursor protein $\alpha$ (sAPP $\alpha$ ), soluble amyloid precursor protein $\beta$ (sAPP $\beta$ ), monocyte chemoattractant protein-1 (MCP1), YKL-40 [63] - may discriminate PSP from PD individuals, as well as CBS from PD and AD/FTD though with conflicting results (Table 3). Similarly, p-tau and t-tau CSF concentrations do not differentiate idiopathic normal pressure hydrocephalus (iNPH), a well-known parkinsonian syndrome-mimic, from PSP [64] (Table 3). Nonetheless, the combination of t-tau, A $\beta 40$ and MCP1 differentiate iNPH from other neurodegenerative movement disorders taken as a whole (e.g. PD, MSA, PSP and CBD irrespective of their underlying pathology) with good accuracy (AuROC: 0.80) $[65]$.

Within the tau-spectrum, a trend towards higher CSF tau (both t-tau and p-tau) levels has been reported in CBS compared to PSP and HCs [66-68]. Accordingly, Aerts and colleagues showed that t-tau and p-tau differentiated CBD from PSP with fair diagnostic accuracy (AuROC: 0.77 and 0.76 , respectively) and that increased tau levels were associated with lower_Mini Mental State Examination (MMSE) scores [69]. These data euld-suggest a potential-role of tau biomarkers in the differential diagnosis of tauopathies and a potential role-in the stratification of different phenotypes (e.g. motor-dominant vs. cognitive-dominant). However, these results should be taken with caution since AD-pathology underpins CBS in about $20 \%$ of cases, thus representing a potential bias for the correct interpretation of CSF tau levels. Additionally, these data came come from small samples, and-patients' selection relyare based on use old-obsolete diagnostic criteria for patient selection $[58,70]$.

Tau isoforms may help in differentiating tauopathies from other neuropathologies and to stratify the tau-spectrum. The quantification of alternative tau fragments compared to the classic ones detected by standard ELISA methods is promising [71]. Borroni and colleagues, showed that 33kDa-tau/55kDa-tau ratio discriminate PSP from HCs (AuROC: 0.90) and other NDDs (e.g. AD, CBD, FTD etc.) with a good to excellent diagnostic accuracy-ranging from good to excellent (AuROC: 0.93 for AD, AuROC: 0.87 for CBD, AuROC: 0.86 for FTD) [68,72]. These authors reported a lower $33 \mathrm{kDa}$-tau $/ 55 \mathrm{kDa}$-tau ratio, as assessed by semiquantitative immunoprecipitation, in patients with PSP compared to other tauopathies and $\underline{\alpha-s y n u c l e i n o p a t h i e s . ~ T h e y ~ a l s o ~ s h o w e d ~ a ~}$ correlation of this ratio with brainstem atrophy and motor impairment in PSP $[68,72]$. Other groups did not replicate these results and further investigations are needed [73]. 
Available commercial kits currently quantify tau concentration targeting its central core region. More recently, alternative tau fragments detected using antibodies recognizing the N-terminal fragment $\tau 12-\mathrm{BT} 2$ has been assessed, showing an excellent diagnostic accuracy in differentiating PSP from AD [74](Table 3). Furthermore, a mass spectrometry-based study_( (MS, see table 1), identified 18 different CSF tau fragments with divergent patterns of expression in PSP compared to $\mathrm{AD}$ and to HCs [71].

In last year the application of RT-QuIC and PCMA technologies may isolate and quantify different tau strains (e.g. 4R and 3R tau) in CSF, thus representing an ideal tool to stratify in vivo $3 \mathrm{R}, 4 \mathrm{R}$ and mixed $3 \mathrm{R} / 4 \mathrm{R}$ tauopathies [41].

Stepping aside from CSF, enly a single study explored the potential discriminating value of plasma p-tau181 in tauopathies, using ultrasensitive detection techniques (Simoa). Plasma p-tau was able to differentiate PSP and CBS from AD with good diagnostic accuracy (Table 2), being associated with A $\beta 42$ pathology assessed by cerebral amyloid-PET examinations [75]. The validation of tau-biomarkers in plasma in large longitudinal cohorts using ultrasensitive techniques is warranted.

Tau biomarkers may have a prognostic context-of-use in 4R-tauopathies. Constantinescu and colleagues showed that t-tau CSF levels may predict mortality in a cohort of PSP patients (see table 3 for the Hazard Ratio) [76]. Increased CSF t-tau levels at baseline are associated with a faster decline as measured by clinical progression scores like the Schwab and England ADL (SEADL); decreased baseline p-tau predicts low SEADL scores and rapid decrease in PSP Rating scale (PSPRS). The prognostic value of tau is increased when combining biomarkers into ratios (e.g., baseline p-tau, t-tau and NFL concentrations) [77]. Currently, the role of tau as prognostic biomarker in 4R-tauopathies is limited and should be clarified in longitudinal studies.

\section{The diagnostic and prognostic role of tau in ALS-FTD spectrum}

Frontotemporal dementia (FTD) and Amyotrophic lateral sclerosis (ALS) are NDDs with significantly clinical and pathological overlaps.

FTD is a heterogeneous cognitive disorder, recognizing multiple clinical and pathological phenotypes $[78,79]$, related to the degeneration of frontal and temporal lobes [78]. Three main clinical variants of FTD have been described: the behavioral variant of FTD (bvFTD) [80], primary progressive aphasia (PPA) and the motor FTD syndromes (CBS and PSP) [81]. PPA can be further divided into a semantic variant (svPPA) and in a non-fluent variant (nfvPPA) [82]; the logopenic variant of PPA (lvPPA) is conversely considered an atypical AD variant rather than a proper FTDLD-syndrome [78]. On the other hand, three main pathological FTD phenotypes have been 
described, namely FTD with TDP-43 aggregates (FTD-TDP), FTD with tau depositions (FTD-Tau) and FTD with FUS aggregates (FTD-FUS) [8]. It should be noticed that the correlation between the clinical and the pathological phenotype is not always predictable. While nfvPPA is associated in most cases with FTD-tau (85\% of all cases) and svPPA with FTD-TDP43 pathology $(90 \%$ of cases), bvFTD can be associated with both FTD-tau and FTD-TDP43 histological patterns [8].

ALS is progressive NDD characterized by motor symptoms related to the progressive degeneration of the upper (UMN) and lower motoneuron (LMN) [83]. ALS is strictly associated to TDP-43 pathology in about $95 \%$ of patients, alternative pathological inclusions (e.g. SOD-1 inclusions) may be reported in specific variants in a minority of cases [83].

The clinical and pathological boundaries between FTD and ALS are blurry and the current diagnostic criteria consider ALS and FTD as a complex clinical continuum and pathologic spectrum as well $[84,85]$.

In fact, ALS patients show cognitive impairment in about $50 \%$ of cases, meeting current criteria for diagnosing dementia in $10-15 \%$ of cases [86], and behavioral impairment in up to 45 $50 \%$ of patients baseline [87]. Conversely, FTD patients may present motor symptoms in approximately $30 \%$ of cases showing motor neuron dysfunction ( $12.5 \%$ of bvFTD patients) [88]. The assessment of Tau as a biomarker in the ALS-FTD spectrum is controversial and recent studies on CSF tau showed conflicting results.

Some authors observed a higher concentration of CSF t-tau in FTD patients compared to HC, distinguishing them with a good diagnostic accuracy [89-91], (see Table 3 for AUROC values). On the other hand, other investigations failed in detecting any significant difference [9295]. These conflicting results may be due to the fact that FTD is a highly heterogeneous diagnostic frame, including different clinical and pathological phenotypes. Inconsistent results have been reported in ALS studies [91,93,96-100].

Regarding the differential diagnosis between FTD and AD, p/t-Tau, t-tau and its ratios with Aß42 may be of clinical interest; FTD patients show lower levels of t-tau [91,94,95,101] and higher of $\mathrm{p} / \mathrm{t}$-Tau [94] when compared to AD patients. Moreover, the ratios between t-tau and A $\beta 42$ are able to distinguish between FTD and AD patients [91,95,101].

The CSF p-Tau and t-Tau ratio (p/t-Tau) is more interesting for the clinical workup. p/t-Tau was observed to be significantly lower in ALS patients [89,96-99] and in FTD patients [89,90,92,102], when compared to HC. Not surprisingly, ALS showed lower levels of CSF p/t-Tau than FTD phenotypes with a likely related tauopathy. This ratio was significantly lower in FTD variants with suspected TDP-related pathology (both patients with autopsy-proven TDP-related pathology and TDP-related mutation carriers) compared to FTD phenotypes with a likely underlying tauopathy 
$[89,90,92,94,102,103]$ (Table 3). Low p/t-Tau may therefore represent a marker of an underpinning TDP-43 pathology. From a pathophysiological point of view this could be due to a lower tau burden in TDP-related compared to tau-related FTLD variants. On the other hand, the different proportion

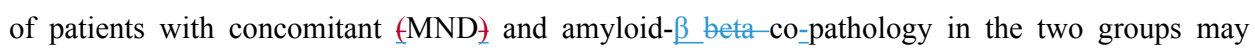
contribute to these observed differences [102]. Furthermore $\mathrm{p} / \mathrm{t}$-Tau ratio seems to correlate with motor cortex thickness and seems to be associated with UMN involvement in ALS [99]. Accordingly the potential role of-p-tau, t-tau andor ef their ratio may find a context--of--use in the in differentiation ofng clinical endophenotypes within the ALS spectrum (e.g. preponderant UMN vs. LMN involvement) but may deserve further investigations are needed.

Different concentrations of CSF tau fragments other than classic tau analytes have been reported by some authors in TDP-related vs. tau-related FTLD [90]. This suggests that differential proteolytic processes may occur in different FTLD variants but this hypotesis and its implications for novel biomarkers research deserves further studies and a more extensive assessment [90].

As regards the role of CSF tau ad a prognostic biomarker, one study showed a potential prognostic value of baseline CSF t-tau in ALS, being a reduction of overall survival observed in individuals with higher CSF t-tau values (Table 3) [97]. Moreover $\mathrm{p} / \mathrm{t}$-tau ratio has been associated with survival in FTD [102] and disease progression in ALS [100].

Moving to surrogated progression biomarkers, a negative correlation between baseline CSF $\mathrm{t}$-tau and the longitudinal increase of ALS Functional Rating Scale Revised (ALSFRS-R) score was described [100]: furthermore $\mathrm{p} / \mathrm{t}$ Tau ratio seems to correlate with motor cortex thickness and seems to be associated with UMN involvement in ALS [95]. Acordingly the toul and of their ratio in differentiating clinieal phenoypes within the ALS spectum (e.g. prependerant UMN is. LMN invelvement) may deserve further investigations.

\section{The diagnostic and prognostic role of tau in alpha-synucleinopathies $\underline{\alpha-s y n u c l e i n o p a t h i e s}$}

Parkinson's disease (PD), Dementia with Lewy Bodies (DLB) and Multiple system atrophy_(MSA) belong to a group of NDDs collectively called synucleinopathies $\underline{\alpha-s y n u c l e i n o p a t h i e s}$. They are pathologically characterized by abnormal deposition of $\alpha$-synuclein forming abnormal depesition such as the ( $\alpha$-syn)-rich neuron intracytoplasmic inclusions (Lewy bodies, Lewy neuritis and glial cytoplasmic inclusions) or the oligodendrocytes cytoplasmic inclusions [104]. Nevertheless, other misfolded proteins have been recognized in degenerated neurons, including tau proteins [105] highlighting the necessity of a more complex pathophysiological model.

In contrast to tau, which seems to require cofactors, in vitro and in vivo studies demonstrated how $\alpha$-syn is able to self-polymerize [106] [107]. Several pathophysiological 
mechanisms have been proposed to explain the interaction between $\alpha$-syn and tau. According to z two-step model (initiation followed by propagation), different models $\alpha$-syn can acts as the "amyloidogenic seed" promoting tau aggregation with subsequent interaction between the two proteins enhancing promoting tau aggregation, whereas a syn and tau interact subsequently to promote each other's fibrillization [108] : or as the pathological chaperone According to another model, a-syn represents the pathological chaperone-for tau fibrillization [109]. Whatever the mechanism, oligomeric forms of $\alpha$-syn and tau co-exist in synucleinopathies $\alpha$-synucleinopathies and influence each other and perpetuate mutual aggregation, leading to hybrid oligomers' formation $[9,110,111]$. Pathogenetic mutations of $\alpha$-syn provide evidence about the role of $\alpha$-syn in promoting the formation of tau inclusions in the human brain. PD patients with $153 \mathrm{~T}$ mutation in the a syn gene (SNCA) revealed abundant $\alpha$-syn and tau inclusions [108]. Nonetheless, tau and $\alpha$-syn inclusions have been also demonstrated in individuals with sporadic neurodegenerative disorders [104].

Interestingly, levels of CSF $\alpha$-syn and p-tau are directly correlated in synucleinepathies $\underline{\alpha-}$ synucleinopathies. This could be because the accumulation of $\alpha$-syn in PD brain could inhibit the release of p-tau in CSF through unknown mechanisms, supporting the relationship between $\alpha$-syn and tau in synucleinepathies $\alpha$-synucleinopathies [112]. With regard to CSF, reduced levels of $\alpha$ syn, t-tau, $\mathbf{p}$-tau and $\beta$-amyloid $\underline{\beta}$ at disease onset have been found in PD compared to HC [112][113]. Furthermore, $p$-tau levels and p-/t-tau ratio increased over time after PD onset despite stable levels of t-tau/ $\alpha$-syn ratio. Patients exhibiting postural instability and gait impairment predominant phenotype had lower CSF p-tau and A $\beta 42$ concentrations than those with tremordominant phenotype [112]. Male sex and SNCA polymorphism rs316181 were linked to increased levels of $\mathrm{p}$-tau, while an increased t-tau/A $\beta 42$ ratio was associated with REM behavior disorder [113].

The diagnostic value of CSF tau biomarkers is higher when they are combined into ratios [112][114][115][113][115]. The p-tau/ $\alpha$-syn ratio (which is increased in PD compared to HC), showed the highest accuracy for PD diagnosis [115] (Table 3). Roughly $75 \%$ of PD patients with a disease course longer than 10 years evolve to PD with dementia (PDD) [116]. CSF t-tau has been reported to be increased in PDD compared to HC, while p-tau did not show differences in the two groups [117]. Longitudinal studies in PD patients found neither a predictive value for the subsequent development of dementia for CSF t-tau and p-tau levels measured at PD onset [118], nor an association with dementia severity in PDD [117]. In contrast, plasma t-tau and p-tau181 levels measured through immunomagnetic reduction (IMR) were increased in DLB compared to HC, and reduced compared to PDD [37] (Table 2). 

belongs to the spectrum of synucleinopathies $\alpha$-synucleinopathies, nevertheless, a mixed neuropathology with amyloid- $\beta$ plaques and tau pathology is present in nearly $40 \%$ of patients $(\alpha-$ syn $+A D)$ and are associated with an increased rate of dementia and a decreased survival time [119]. Although previous studies reported increased levels of t-tau in DLB compared to HC [120], these findings were not replicated in more recent studies [121]. The use of different diagnostic criteria and populations may in part explain these inconsistent results. In general, CSF t-tau and p-tau levels are lower in DLB than AD [120], suggesting that levels of p-tau in CSF could represent a valuable marker for the differential diagnosis between these two NDDs [123]. On the other hand, t-tau levels are higher in DLB in comparison to PD and PDD, supporting the hypothesis that limbic and cortical Lewy body pathology is the main and specific pathologic correlate of dementia in PD [124]. In a neuropathological study, the regional distribution of tau and amyloid- $\beta$ is different in $\alpha-\operatorname{syn}+\mathrm{AD}$ patients and $\mathrm{AD}$, with a greater proportion of tau in the temporal neocortex for DLB patients and in frontal neocortex in AD. Moreover, the severity of tau burden was correlated with worse antemortem cognitive performances in DLB individuals [125]. Tau pathology has also been correlated to the pattern of brain atrophy in MRI. Indeed, increased CSF t-tau levels were associated with high global atrophy scores [126] and elevated CSF p-tau with a more selective posterior atrophy [127]. $\mathrm{CSF}$ ratios t-tau/A $\beta 42$ and $\mathrm{p}$-tau/A $\beta 42$ recently proved to predict the presence of $\mathrm{AD}$ co-pathology in DLB, with higher levels of both ratios in $\alpha$-syn+AD brains when compared to $\alpha$-syn-AD brains, while no correlation was found analyzing single biomarkers. Besides, higher CSF t-tau/A $\beta 42$ and lower CSF A $\beta 42$ levels were associated with a higher burden of neocortical $\alpha$-syn [121].

MSA is a NDD characterized by autonomic failure associated with a poorly levodoparesponsive parkinsonian syndrome or cerebellar ataxia, thus defining two main phenotypes respectively identified as MSA-P and MSA-C [128,129]. Differential diagnosis between PD and MSA could be challenging because of the clinical overlap in the early stages and a reliable biomarker for the differential diagnosis of MSA from PD yet represents an unmet need. Reeluced CSF levels $\alpha$-syn in MSA patients compared to HC have been reported in most studies, while results on blood samples were inconclusive [111]. AlsoD, data on CSF biomarkers in MSA are conflictingen tau are conflicting. In some studies, CSF t-tau concentration was higher in MSA than PD patients or HC [130][131], whereas others reported a reduction of its level [132]. By contrast, ptau concentration was similar in MSA and HC [130][132]. Conflicting data have been published on the p-tau/t-tau ratio, reported either higher [130][132] or lower [133][134] in MSA compared to PD. The combination of p-tau181, t-tau and DJ1 (a protein related to PD pathogenesis whose function is still unknown in MSA) proved high sensitivity and specificity (respectively $82 \%$ and $81 \%$ ) to 
discriminate MSA from PD [131], as well as the p-tau/A $\beta 42$ ratio (specificity $71 \%$, sensibility 93\%), which is significantly increased in MSA [134] (Table 3). Similarly, the AD index (CSF $\mathrm{A} \beta 40 / 42$ ratio $\times \mathrm{t}$-tau) and the ratio $\mathrm{t}$-tau $/ \alpha$-syn were higher in MSA than $\mathrm{HC}$, while no significant differences were found comparing PD and HC [130]. Finally, no CSF biomarkers proved to be reliable for the differential diagnosis between MSA-C and MSA-P [114].

Fluid biomarkers for synucleinopathies $\alpha$-synucleinopathies were recently investigated also in blood. Red blood cells (RBC) seem to be a preferential niche for misfolded proteins [135] and oligomeric $\alpha$-syn was described in RBC of patients with PD [136]. Analysis of RBC in PD patients showed reduced levels of $\alpha$-syn and increased levels of phospho-tau (p-tau) in PD compared to HC [110]. Interestingly, RBC t-tau concentration was inversely correlated to MMSE scores both in the general PD cohort and in PD drug naïve patients, suggesting a central role of tau pathology in promoting cognitive decline in PD [110]. Plasma t-tau and p-tau were higher in MSA compared to HC when measured through IMR [137][37], while levels of p-tau measured through Simoa did not differ between MSA and HC [24].

\section{Tau PET in Tauopathies}

In the field of neurodegenerative dementias, in vivo imaging with radioligands designed to detect each underlying proteinopathy aims to find a gold standard biological marker. Amyloid- $\beta$ imaging has been incorporated into AD diagnostic criteria since 2007 [138]. However, cerebral amyloid-PET uptake did not correlate with cognitive performance nor neurodegeneration in AD. Biomarkers of tau pathology may be closely related to neuronal injury and changes in cognition. Tau-PET will support the investigation of the spatial-temporal evolution of tau pathophysiology in $\mathrm{AD}$ and other NDD as well as the clinical validation of fluid biomarkers. Therefore, research effort is focusing on the development of tau-selective positron emission tomegraphy (PET) radiotracers in order to reveal underlying tau deposits in a broad range of tauopathies, such as AD, FTD, PSP. A significant number of tau PET tracers have been synthesized so far, but many of those lack sufficient specificity and selectivity $[139,140]$. The main difficulties to develop these tracers are related to [141]: 1) lipophilicity, which is necessary to cross the blood-brain barrier and the cell membrane (tau is located both intra and extracellularly); by contrast, excessive lipophilicity could lead to unspecific binding; 2) affinity for tau, relevant to obtain high selectivity for tau molecule and overcoming the high $\mathrm{A} \beta$ concentrations interfering with tau-ligand binding, but requiring prolonged scanning time to reach the steady-state; 3 ) different conformations of tau aggregates and different tau isoforms [142], making it challenging to develop a unique tau radiotracer for all types of tauopathies. Based on both in vitro and in vivo results, three families of radiotracers were 
initially synthesized: 1) the THK family (THK117, its (S)-enantiomer THK5317, and THK5351; 2) 18F-AV1451, also known as T807 or Flortaucipir; 3) PBB3. The so-called first-generation tau tracers finally showed a lack of specificity, with relevant off-target binding to monoamine oxidase B (MAO-B) for the HK family and Flortaucipir [143], neuromelanin for Flortaucipir [144], and A $\beta$-plaques and $\alpha$-syn for PBB3 [145,146]. Hence, the second generation of tau PET tracers has been developed: RO948, PI2620, JNJ311, MK6240, PM-PBB3 and AM-PBB3 [147]. However, the most widely studied agent is [18F]AV-1451 (Flortaucipir). It was the first approved tau PET tracer for estimation of the density and distribution of aggregated tau neurofibrillary tangles in adults with cognitive impairment and suspected AD [148].

\subsection{Aging}

First-generation tracers demonstrated retention of uptake confined to the medial temporal lobe in normal cognitively elderly, in accordance with pathological data [149], likely reflecting an agerelated process of tau-deposition [150]. Accumulation of tau in the temporal cortex, without an associated significant $A \beta$ burden, may suggest a promoting effect of tau in mild amnestic deficits; hippocampal atrophy or a PART related pathology in such subjects.

\subsection{Alzheimer's Disease}

There is now convincing evidence that cortical tau binding at PET imaging reflects tau accumulation reported from histopathological studies in AD brains [149]. 18F-AV1451 demonstrated high affinity in vitro for paired helical filaments (PHF) of 3R/4R tau isoforms reported in AD brains [151,152]. A recent study in 82 individuals with or without dementia showed a high concordance between the visual reading of 18F-AV1451 PET scans and staging of cortical neurofibrillary tangles, reinforcing the concept that Flortaucipir effectively reflects pathological changes of AD [153]. Similar results were replicated in vivo, with 18F-AV1451 demonstrating to accurately differentiate clinically diagnosed AD from $\mathrm{HC}$ and other NDDs [154]. Higher levels of tau tracer retention in the inferior lateral temporal region, but also in the posterior cingulate and lateral parietal regions, provided the best discrimination areas between

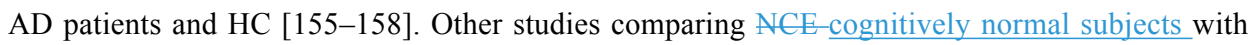
MCI patients showed differences in binding restricted to medial temporal regions (parahippocampal and entorhinal cortex) [159,160], as well as lateral temporal and parietal areas when examining only amyloid- $\beta$-positive MCI individuals [158]. Furthermore, some studies reported greater retention of $18 \mathrm{~F}-\mathrm{AV} 1451$ in the cortex of younger compared to older $\mathrm{AD}$ patients, with a cut off of 75 years-old [161], and in early-onset compared to late-onset AD 
patients with a cut off of 65 years-old [160], as similarly reported in previous post-mortem studies [162]. Moreover, neocortical Flortaucipir retention was found in preclinical AD cases and rarely in amyloid- $\underline{\beta}$ negative cases $[161,163]$.

The extent of tau accumulation intimately correlates with the severity of MCI due to AD and AD dementia, providing an objective index for disease severity. The few available longitudinal tau PET studies in AD demonstrated a tracer retention related to disease duration [164-167]. However, results from the largest studies, which compared cognitively unimpaired $A \beta$-negative and $\mathrm{A} \beta$-positive individuals, $\mathrm{MCI}$ and $\mathrm{AD}$ dementia patients, are conflicting. Cho and colleagues studied 107 participants (45 A $\beta$-negative cognitively unimpaired, 7 A $\beta$-positive cognitively unimpaired, $31 \mathrm{MCI}$, and $24 \mathrm{AD}$ dementia) who completed both $18 \mathrm{~F}$-flortaucipir and $18 \mathrm{~F}$ florbetaben at baseline and who were followed up for 2 years. The authors found a predominant tau accumulation in the medial and basal temporal cortices in MCI and in the lateral temporal cortices in AD dementia [167], thus supporting a progressive tau accumulation pattern in line with the Braak model [149]. Conversely, Jack and colleagues evaluated the longitudinal change in tau PET signal during a one-year follow-up in a group of 126 individuals (59 cognitively unimpaired $A \beta$-negative, 37 cognitively unimpaired $A \beta$-positive, and 30 cognitively impaired $A \beta$ positive). They demonstrated a tau accumulation in the A $\beta$-positive clinically unimpaired group in the medial temporal lobe and in the medial parietal areas, including posterior cingulate cortex. This suggests that initial accumulation of tau aggregates in $\mathrm{AD}$ may not be restricted to the medial temporal lobes as implied by Braak staging [165]. Overall these evidence proved that longitudinal tau PET may be an useful biomarker in clinical trials to monitor the effect of diseasemodifying therapies tailored to reduce tau as well as cerebral amyloid- $\beta$ plaque burden [168]. In AD increased 18F-AV1451 uptake is strongly co-localized with hypometabolic regions and has been associated with worse performance on various cognitive domains in regionally specific patterns [169]. Accordingly, 18F-AV1451 distribution may predict the clinical variants of AD suggesting on-site neurotoxicity provoked by tau aggregates in posterior cortical atrophy, logopenic variant of primary progressive aphasia, or behavioral/dysexecutive variant of AD [169172]. The main dare challenge for tau tracers remains the capability to detect preclinical / prodromal / early AD stages. Importantly, the sensitivity for an early diagnosis is limited by the fact that in the early AD stages (e.g. MCI) neurofibrillary tangles are only present in deep brain regions. In more advanced AD stages $18 \mathrm{~F}$-AV1451 discriminate mild-to-moderate AD patients from $\mathrm{HC}$ [154].

Currently, there are not conclusive studies on second-generation tau tracers in AD patients and we do not have any head-to-head comparison between them. Available studies suggest that they may 
be more useful in identifying earlier Braak stages. They also show substantially less non-specific binding or higher affinities to primary tauopathies [173]. These agents include [18F]MK-6240 (Merck \& Co), PM-PBB3 (APRINOIA Therapeutics), F18-PI-2620 (Life Sciences) (50) and RO-948 (Roche). RO-948 tracer showed similar patterns of cerebral uptake then 18F-AV1451 [174]. Moreover, RO-948 showed a greater stability, a higher retention in the medial temporal lobe and lower intracerebral "off-target" binding than Flortaucipir [175,176]. In AD subjects, PET images of F18-PI-2620 showed a significantly higher uptake than control subjects in temporal lobe, parietal and cingulate cortex. Importantly, in non-demented control subjects it showed robust initial brain uptake and fast washout from the brain, as well as no age dependency. This last finding suggests that F18-PI-2620 could improve the discrimination between nondemented control and AD subjects in elderly. Moreover, excellent test-retest variability has been demonstrated confirming the utility of F18-PI-2620 to evaluate longitudinal change of tau deposition during disease course [177]. Finally, three recently published studies have evaluated [18F]MK-6240 in vivo [178-180]. All these studies exhibited favorable kinetic and high binding levels of [18F]MK-6240 to brain regions typically affected from NFT deposition in AD subjects. [18F]MK-6240 PET images in another cohort of patients positive for cerebral amyloid-ßbeta deposition (cognitive unimpaired/impaired elderly controls, MCI and AD patients) showed a pattern corresponding to the anatomic distribution of tau as expected in the Braak model [178].

\subsection{Non-AD tauopathies}

Tau imaging may also be relevant for other tauopathies, such as CBD and PSP. These atypical parkinsonian syndromes are characterized by abundant filamentous tau inclusions that are made of isoforms with four microtubule-binding repeats in tubular or straight filaments. In PSP and CBD abnormal aggregation of pathologically misfolded and hyperphosphorylated tau proteins mainly occur in the basal ganglia in the early stages, spreading later to multiple brain areas: brainstem, posterior frontal lobe, cerebellum and association cortices for PSP; primary motor cortex, frontal lobe, brainstem or cerebellum for CBD [181-184]. Nevertheless, a variety of overlapping syndromes are frequent in tauopathies.

To date few studies using low specific first-generation PET tracers have examined tau binding in parkinsonian disorders. They revealed distinct patterns of tracer retention in PSP and CBD, compared to controls, showing elevated PET signal in the basal ganglia and affected cortical regions, in agreement with postmortem data [185]. 
Elevated tau deposition in PSP cases was observed in the basal ganglia, thalamus, dentate nucleus of the cerebellum, and midbrain [186-192]. However, an extensive overlap and age-dependent increase in both the PSP and control groups has been reported [187]. Significant differences in 18F-AV-1451 distribution between patients with PD and PSP, with increased uptake in the globus pallidus, midbrain, and subthalamus in PSP cases, have been also described [193,194]. Interestingly, in some studies the radioligand accumulation correlated with the clinical disease severity $[189,195,196]$, but the lack of correlations between tau binding and symptom severity was observed in the other cohorts $[188,194]$. A recent study by Whitwell and colleagues using flortaucipir reported that the clinical heterogeneity present in PSP is mirrored by anatomical and tau burden heterogeneity within the brain [197].

Studies analyzing the feasibility of tau PET in patients with CBS showed elevated asymmetrical tracer deposition in dentate nucleus of the cerebellum, midbrain, subthalamic nucleus, globus pallidus and putamen, precentral and postcentral cortex, superior frontal and parietal lobe, fitting with the regional distribution of tau pathology $[198,199]$. Furthermore, longitudinal investigations documented a correlation between tau accumulation over time and disease progression [166,200]. However, the degree of uptake in CBS using currently available tau ligands is variable, and some patients resulted negative $[201,202]$.

Overall, studies using in vivo tau PET in PSP and CBD individuals that received a postmortem confirmation have shown conflicting results questioning the usefulness of tau imaging in early diagnosis of such conditions. Indeed, the available tracers are not specific for $4 \mathrm{R}$ tau pathology and show substantial off-target binding in the midbrain and basal ganglia to monoamine oxidase B (MAO B) and to neuromelanin-containing cells [192,203].

Second generation of tracers with improved off-target binding are under evaluation also in nonAD with suspected PSP using 18F-PI-2620 which proved high affinity to recombinant 4R tau fibrils and PSP brain homogenate [204,205].

\section{Discussion and perspectives}

Epidemiological projections indicate that AD evolving epidemic represents a global threat for healthcare systems [54]. Minimally invasive and globally accessible tests are needed to cope with this expected burdensome demand and to manage individuals in suspected preclinical/prodromal stages of NDDs [206,207]. Blood-based biomarkers represent cost-, resource- and time effective tools [208]. They hold the potential to enable large-scale biological screening of individuals who are very unlikely to have AD-related pathophysiology and would support the request of second- 
level investigations (e.g. PET imaging or CSF assessment). Blood-based biomarkers not only open up the opportunity of a multi-step diagnostic work-up but can also facilitate the reengineering of drug Research \& Development (R\&D) pipelines, from subjects' enrollment, target engagement, to treatment efficacy monitoring. In Oncology, serial liquid biopsies offer clues about the evolution of cancer in individual patients across disease stages, enabling individualized genetically and biologically guided therapies [207].

The development of novel ultrasensitive measurement techniques has enabled the detection of tau-related biomarkers, facilitating a liquid biopsy-driven paradigm shift in the field of NDDs, including Alzheimer's diseaseAD [207]. Plasma t-tau seems to have a limited utility as diagnostic marker in NDDs when assessed alone [47]. On the other hand, plasma p-tau may find a context-ofuse in the differential diagnosis between $\mathrm{AD}$ and non- $\mathrm{AD}$ dementias, including primary tauopathies and vascular dementia $[25-29,75]$. The combination of tau with other putative biomarkers (e.g. NFL) into panels or ratios seems to increase the diagnostic accuracy of tau biomarkers alone [22]. Sets of multiple biomarkers may accordingly find a context-of-use in the clinical diagnostic workup of neurodegenerative dementias and, in patients' selection and their follow-up for targetedtherapiesdisease modifying clinical trials.

Studies investigating p-tau isoforms other than p-tau181 (e.g. p-tau217) [30,33,209], and alternative proteolytic fragments like the N1-tau [25] fragment have shown encouraging results in differentiating $\mathrm{AD}$ and $\mathrm{MCI}$ from $\mathrm{HC}$ and in predicting the amyloid $\beta$ - in preclinical $\mathrm{AD}$. Several studies, performed in AD and NDDs, indicate that plasma p-tau217 is a reliable predictor of tau pathology (as assessed through tau-PET) [210], amyloid- $\beta$-mediated tau pathophysiology, longitudinal cortical/subcortical atrophy and AD-like cognitive decline. These findings coupled with the evidence that p-tau217 discriminates AD from non-AD conditions [30,31] explain why plasma p-tau217 will be used as exploratory marker in different COU, including patients' selection and follow-up in clinical trials. The evidence about plasma p-tau217 also support its integration in the ATN matrix for disease diagnosis, prognosis and progression monitoring in clinical practice $[31]$.

not yet available A better understanding of time-trajectories of these alternative phosphorylation sites of p-tau mays mandatory to help understand the more adequate context-ofuse of each isoform from preclinical to full-blown AD.

Tau protein misfolding and accumulation in toxic species and tangles is a critical pathophysiological process of $\mathrm{AD}$ and of primary tauopathies [1]; further, this protein contributes 
to the pathophysiology of other brain proteinopathies (e.g. a synuclein, TDP 43, FUS), leading to mixed or overlapping neuropathologies [203].

Biomarkers tracking in vivo tau pathology are embedded in the most recent 2011 NIA-AA and 2014 IWG 2 diagnostic criteria for AD, thus representing key elements for the diagnestic workup of $\mathrm{AD}[11,135]$. Currently CSF total-tau, 181phosphorylated-tau, and tau-molecular imaging (PET), represent validated biomarkers for investigating tat-related pathology in $A D$ $[11,135]$.

However, CSF assessment through lumbar puncture and PET assessment through radiotracers and imaging device are invasive, time and resource demanding.

According to recent investigations plasma t tau seems to have a limited utility as diagnostic marker in NDDs when assessed alone [46]. On the other hand, plasma p-tau help differentiate AD from non-AD dementias, including primary taupathies and vascular dementia [24-28,71]. The combination of plasma p tau and t tau into ratios with other candidate biomarkers (e.g. NFL) seems anyway to increase the diagnostic power of these analytes [22]. Studies investigating $\mathrm{p}$ tau isoforms other than p-tau181 (e.g. 217p-tau) [29,32,204], and alternative proteolytic fragments like the N1tau [24] fragment have shown encouraging results in differentiating $\mathrm{AD}$ and $\mathrm{MCI}$ from $\mathrm{HC}$ but these data should be regarded with caution and may deserve a more extensive validation.

In primary tauopathies CSF p-tau and t-tau, as assessed by traditional techniques, are not reliable biomarkers for the diagnostic workup of 4R-tauopathies. In fact, both CSF t-tau and $\mathrm{p}$-tau and their combination do not discriminate primary taupathies from $\mathrm{HC}$ and the tau related phenotypes within the pathologic tau-spectrum [64]. These biomarkers are useful in both clinical and research settings for differentiating NDDs with an underlying AD pathology, which can mimic primary tauopathies $[59,60]$. The detection of different tau isoforms and fragments (e.g. $33 \mathrm{KDa}$ vs $55 \mathrm{KDa}$ isoforms) [72] and of specific tau strains (e.g. 3R and 4R tau) by means of PMCA and RTQuIC [41] are promising strategies-tools to stratify the pathologic tau-spectrum and its clinical eontinuum. to identify different phenotypes. The prognostic value of tau biomarkers for in the 4Rtauopathies remains to be clarified, especially when measured in plasma with the use of novel ultrasensitive techniques, which allow minimally invasive and repeatable examinations.

As regardsIn the ALS-FTD spectrum, CSF t-tau and p-tau may contribute in discriminating AD from FTD and in identifying patients with FTD phenotypes but with an underlying ADpathology [91]. The diagnostic value of p-tau and t-tau alone in differentiating the FTD-ALS continuum from healthy contrels $\underline{\mathrm{HCs}}$ is controversial $[89,91,98]$. Nevertheless p-tau/t-tau ratio seems to be tower in FTD and ALS compared to HC and may indirectly indicateuseful in FTD for the identification of patients with a TDP-43 underlying pathology related variants [89]_- and may be 
useful in ALS to discriminated clinical endophenotypes (UMN-predominant vs. LMN-predominant variants) [99]. A possible explanation could be the lower cerebral tau burden in TDP-43 related FTD-ALS phenotypes. Baseline CSF t-tau and p-tau/t-tau ratio could represent a prognostic marker for both FTD and ALS [97] but further studies are needed to identify the proper context-of-use of these biomarkers.

In the reported studies, the high heterogeneity of the clinical diagnostic criteria used for patients' selection, the lack of post mortem validation, and the unpredictable relationship between phenotypes and underlying pathology hamper the understanding of tau protein role as NDD biomarker.

In $\alpha$-synucleinopathies the co-occurrence of underlying tauopathy is commonly reported-and tau may have a permissive role on a-synuclein aggregation. Reduced levels of t-tau have been reported in PD compared to HC $[108,109]$, while $\mathrm{p}$-tau levels and $\mathrm{p}$-/t-tau ratio seems to increase ever time [109]. T-tau and p-tau concentration may vary according to PD phenotypes, thus suggesting a potential role of tau in differentiating clinical phenotypes (it is reported a reduction in CSF p-tau levels in patient with postural instability and gait disorder and an increase in patients with REM sleep behavior disorder) [112,113]. An potential-association between tau burden and cognitive impairment in PDD and DLB has been postulated nevertheless but data are conflicting and still inconclusive [117,118]. Similarly the diagnostic value of CSF p-tau and t-tau in atypical parkinsonism (e.g. MSA and DLB) is controversial $[117,121,122,124,132,211][130,131]$.

Recent investigations on plasma reported an increase of p tau concentration in PD discriminating PD from HC, and inversely correlated with MMSE scores. Further studies are needed to establish the diagnostic and prognostic value of both $p$-tau and $t$-tau in $\alpha$ synucleinopathies and their role in differentiating PD phenotypes.

The development and validation of $\underline{\text { As regards }}$ tau-PET tracers ${ }_{2}$ tau for the in vivo tracking of tau pathology has posed several challenging questions [138]. Tau-PET tracer uptake has been included in the current diagnostic criteria for $A D$ as a surrogated marker of tau accumulation [12]. Tau accumulation in the medial and basal temporal cortices was observed in MCI patients-was demonstrated, thus suggesting a potential role of tau-PET tracers in the prodromal AD and a potential value of tau-PET uptake as a biomarker tein monitoring the efficacyect of diseasemodifying therapies in clinical trials [165,167]. Further longitudinal observations are required to corroborate this hypothesis. A possible role of tau-PET imaging in the differential diagnosis between $4 \mathrm{R}$ tauopathies is suggested by some studies highlighting a different deposition pattern of tau in PSP compared to CBS. Off-target binding in the midbrain and basal ganglia is currently limiting the use of fist generation tracers but This issue-was partly resolved with the 
development of second-generation tau tracers [145]. Further evidence on the physiological and pathologic variables influencing tau-PET tracers uptake, on the off-label binding and on the comparison of different tracers is warranted to understand the appropriate context-of-use of these biomarkers.

10. Conclusions and perspectives

$\underline{\text { In conclusions, biomarkers development and related research have significantly }}$ contributed to the reengineering of $\mathrm{AD}$ as a clinical-biological framework, as reflected by the ATN system, where preclinical stages of the disease are identified and potentially treated for preventive strategies, including tau-targeting approaches. It is conceivable that the same paradigmng shift may take place also for NDD others than AD. Individuals with no or subtle eognitive/mothehavioral declinedifferent clinical presentations but similar biomarkers may be grouped iento biological clusters facilitating a treatment essentialyessentially based on pathophysiological mechanisms and not phenotypes to treat with pathway-based, symptom (i.e., NDD clinical phenotype) agnostic therapies $[212,213]$, in line with the precision medicine paradigm [214]

Epidemiological projections indicate that $\mathrm{AD}$ evolving epidemic represents a global threat for healthcare systems, especially if a disease-modifying therapy becomes available [206]. Minimally invasive and globally accessible tests are needed to cope with this expected burdensome demand and to manage individuals in suspected preclinical/prodromal stages of NDDs, including Alzheimer's [207,208]. Blood based biomarkers represent cost, resource- and time effective tools for critical clinical solutions [209]. They hold the potential to enable large seale biological screening of individuals who are very unlikely to have AD-related pathophysiology and would support the request of second-level investigations (e.g. positron emission tomography [PET] imaging or cerebral-spinal fluid [CSF] assessment) that have reduced accessibility and are more invasive. Blood-based biomarkers not only open up the opportunity of a multi-step diagnostic work-up but can also facilitate the re-engineering of drug Research \& Development (R\&D) pipelines, from subjects' enrollment, target engagement, to treatment efficacy monitoring. In Oncology, serial liquid biopsies offer clues about the evolution of cancer in individual patients across disease stages, enabling individualized genetically and biologically guided therapies [208].

The development of novel ultrasensitive measurement techniques has enabled the detection of tau related (and others) biomakkers, facilitating a liquid biopsy driven paradigm shift in the field of neurodegenerative diseases, including Alzheimer's disease [208]. 

contributed to the reengineering of $\mathrm{AD}$ as a clinical-biological framework, as reflected by the ATN system, where preclinical stages of the disease are identified and potentially treated for preventive strategies, including tau targeting approaches.

It is conceivable that the same parading shift may take place also for NDD others than $\mathrm{AD}$. Individuals with no or subtle cognitive/motor/behavioral decline may be grouped onto biological elusters to treat with pathway based, symptom (i.e., NDD - clinical phenotype) agnostic therapies[210,211], in line with the precision medicine paradigm [212].

\section{Expert opinion}

Tau, as assessed in CSF in the form of t-tau and p-tau181, is an established diagnostic biomarker for AD.- The detection of tau in alternative easily accessible matrices like plasma currently represents one of the most exciting future directions for $\mathrm{AD}$ biomarker research. The further development of ultrasensitive measurement techniques and their more extensive validation represent accordingly a hot topic in this field. The potential utility of alternative tau fragments and tau isoforms represents another challenge to improve $\Lambda \mathrm{D}$ diagnosis and stratification.

As regards primary tauopathies, alternative tau isoforms (e.g. 217p-taup-tau217) or proteolytic fragments (e.g. N-terminal fragments) quantified in CSF and in blood may represent candidate fluid diagnostic and prognostic markers overcoming the elassic limitations of current CSF $\mathrm{t}$-tau and p-tau measurement in these conditionsNDDs.

In particular plasma p-tau217 could be used as exploratory marker in different drug development pipelines with distinct molecular target and for different COU, including patient selection, and theragnostic, and potently target engagement. The evidence about plasma p-tau217 also support its integration in the ATN matrix for disease diagnosis, prognosis and progression monitoring in clinical practice.

Nevertheless, the implementation of techniques enabling the identification of different tau strains (e.g. 3R-vs. 4R-tau), enabled by RT-QuIC and PMCA-techniques, represents the most important frontier for fluid biomarker discovery in tauopathies.

The validation of biomarkers tracking tau pathology in the ALS-FTD spectrum is challenging. This could be also- ${ }_{2}$ due to the high clinical and phenotypical heterogeneity intrinsic for this pathologieof this spectrum. However, tau biomarkers may identify AD-related pathology, and, to a lesser extent, differentiate tau-related from TDP-43 related pathology. 


\section{Funding}

The study was not funded. Academic study without external financial support.

\section{Financial disclosures}

The authors have no relevant affiliations or financial involvement with any organization or entity with a financial interest in or financial conflict with the subject matter or materials discussed in the manuscript. 


\section{Bibliography}

[1] Spillantini MG, Goedert M. Tau pathology and neurodegeneration. Lancet Neurol [Internet]. 2013;12:609-622. Available from: http://dx.doi.org/10.1016/S1474-4422(13)70090-5.

[2] Rösler TW, Tayaranian Marvian A, Brendel M, et al. Four-repeat tauopathies [Internet]. Prog. Neurobiol. Elsevier Ltd; 2019 [cited 2020 Nov 22]. Available from: https://pubmed.ncbi.nlm.nih.gov/31238088/.

[3] Höglinger GU, Respondek G, Kovacs GG. New classification of tauopathies. Rev Neurol (Paris). 2018;174:664-668.

[4] Goedert M, Eisenberg DS, Crowther RA. Propagation of Tau Aggregates and Neurodegeneration. Annu Rev Neurosci [Internet]. 2017 [cited 2020 Nov 22];40:189-210. Available from: https://pubmed.ncbi.nlm.nih.gov/28772101/.

[5] La-rocque S De, Moretto E, Schiavo G. Knockin' on heaven 's door : Molecular mechanisms of neuronal tau uptake. 2020;1-26.

[6] Irwin DJ. Tauopathies as clinicopathological entities. Park Relat Disord [Internet]. 2016;22:S29-S33. Available from: http://dx.doi.org/10.1016/j.parkreldis.2015.09.020.

[7] Braak H, Zetterberg H, Del Tredici K, et al. Intraneuronal tau aggregation precedes diffuse plaque deposition, but amyloid- $\beta$ changes occur before increases of tau in cerebrospinal fluid. Acta Neuropathol. 2013;126:631-641.

[8] Elahi FM, Miller BL. A clinicopathological approach to the diagnosis of dementia. Nat Rev Neurol. 2017;13:457-476.

[9] Sengupta U, Guerrero-Muñoz MJ, Castillo-Carranza DL, et al. Pathological Interface between Oligomeric Alpha-Synuclein and Tau in Synucleinopathies. Biol Psychiatry [Internet]. 2015 [cited 2020 Oct 8];78:672-683. Available from: https://pubmed.ncbi.nlm.nih.gov/25676491/.

[10] Henderson MX, Sengupta M, Trojanowski JQ, et al. Alzheimer's disease tau is a prominent pathology in LRRK2 Parkinson's disease. Acta Neuropathol Commun. 2019;7:1-16.

[11] Jack CR, Albert MS, Knopman DS, et al. Introduction to the recommendations from the National Institute on Aging-Alzheimer's Association workgroups on diagnostic guidelines for Alzheimer's disease. Alzheimer's Dement [Internet]. 2011;7:257-262. Available from: http://dx.doi.org/10.1016/j.jalz.2011.03.004.

[12] Dubois B, Feldman HH, Jacova C, et al. Advancing research diagnostic criteria for Alzheimer's disease: The IWG-2 criteria. Lancet Neurol. 2014.

[13] Jack CR, Bennett DA, Blennow K, et al. NIA-AA Research Framework: Toward a biological definition of Alzheimer's disease. Alzheimer's Dement [Internet]. 2018;14:535- 
562. Available from: https://doi.org/10.1016/j.jalz.2018.02.018.

\section{** Recommandations for a biomarker-based diagnosis of AD}

[14] Baldacci F, Mazzucchi S, Della Vecchia A, et al. The path to biomarker-based diagnostic criteria for the spectrum of neurodegenerative diseases [Internet]. Expert Rev. Mol. Diagn. Taylor and Francis Ltd; 2020 [cited 2020 Oct 8]. p. 421-441. Available from: https://pubmed.ncbi.nlm.nih.gov/32066283/.

[15] Baldacci F, Lista S, Vergallo A, et al. A frontline defense against neurodegenerative diseases:the development of early disease detection methods. Expert Rev Mol Diagn [Internet]. 2019;19:559-563. Available from: https://doi.org/10.1080/14737159.2019.1627202.

[16] Xia J, Broadhurst DI, Wilson M, et al. Translational biomarker discovery in clinical metabolomics: An introductory tutorial. Metabolomics. 2013;9:280-299.

[17] Kawarabayashi T, Nakamura T, Miyashita K, et al. Novel ELISAs to measure total and phosphorylated tau in cerebrospinal fluid. Neurosci Lett [Internet]. 2020;722:134826. Available from: https://doi.org/10.1016/j.neulet.2020.134826.

[18] Blennow K, Zetterberg H. Biomarkers for Alzheimer's disease: current status and prospects for the future. J. Intern. Med. 2018.

[19] Saijo E, Metrick MA, Koga S, et al. 4-Repeat tau seeds and templating subtypes as brain and CSF biomarkers of frontotemporal lobar degeneration. Acta Neuropathol [Internet]. 2020;139:63-77. Available from: https://doi.org/10.1007/s00401-019-02080-2.

[20] Hampel H, O’Bryant SE, Molinuevo JL, et al. Blood-based biomarkers for Alzheimer disease: mapping the road to the clinic. Nat. Rev. Neurol. 2018.

[21] Del Prete E, Beatino MF, Campese N, et al. Fluid Candidate Biomarkers for Alzheimer's Disease: A Precision Medicine Approach. J Pers Med [Internet]. 2020;10:221. Available from: https://www.mdpi.com/2075-4426/10/4/221.

[22] Park JC, Han SH, Yi D, et al. Plasma tau/amyloid- $\beta$ 1-42 ratio predicts brain tau deposition and neurodegeneration in Alzheimer's disease. Brain [Internet]. 2019 [cited 2020 Nov 22];142:771-786. Available from: https://pubmed.ncbi.nlm.nih.gov/30668647/.

[23] Karikari TK, Pascoal TA, Ashton NJ, et al. Blood phosphorylated tau 181 as a biomarker for Alzheimer 's disease : a diagnostic performance and prediction modelling study using data from four prospective cohorts. 2020;19.

[24] Karikari TK, Pascoal TA, Ashton NJ, et al. Blood phosphorylated tau 181 as a biomarker for Alzheimer's disease: a diagnostic performance and prediction modelling study using data from four prospective cohorts. Lancet Neurol. 2020;19:422-433. 
[25] Chen Z, Mengel D, Keshavan A, et al. Learnings about the complexity of extracellular tau aid development of a blood-based screen for Alzheimer's disease. Alzheimer's Dement [Internet]. 2019 [cited 2020 Nov 22];15:487-496. Available from: https://pubmed.ncbi.nlm.nih.gov/30419228/.

[26] Palmqvist S, Janelidze S, Stomrud E, et al. Performance of Fully Automated Plasma Assays as Screening Tests for Alzheimer Disease-Related $\beta$-Amyloid Status. JAMA Neurol [Internet]. 2019 [cited 2020 Nov 22];76:1060-1069. Available from: https://pubmed.ncbi.nlm.nih.gov/31233127/.

[27] Mielke MM, Hagen CE, Xu J, et al. Plasma phospho-tau181 increases with Alzheimer's disease clinical severity and is associated with tau- and amyloid-positron emission tomography. Alzheimer's Dement [Internet]. 2018 [cited 2020 Nov 22];14:989-997. Available from: https://pubmed.ncbi.nlm.nih.gov/29626426/.

[28] Janelidze S, Mattsson N, Palmqvist S, et al. Plasma P-tau181 in Alzheimer's disease: relationship to other biomarkers, differential diagnosis, neuropathology and longitudinal progression to Alzheimer's dementia. Nat Med [Internet]. 2020 [cited 2020 Nov 22];26:379386. Available from: https://pubmed.ncbi.nlm.nih.gov/32123385/.

[29] Thijssen EH, La Joie R, Wolf A, et al. Diagnostic value of plasma phosphorylated tau181 in Alzheimer's disease and frontotemporal lobar degeneration. Nat Med [Internet]. 2020 [cited 2020 Nov 22];26:387-397. Available from: https://pubmed.ncbi.nlm.nih.gov/32123386/.

[30] Palmqvist S, Janelidze S, Quiroz YT, et al. Discriminative Accuracy of Plasma Phosphotau217 for Alzheimer Disease vs Other Neurodegenerative Disorders. JAMA - J Am Med Assoc. 2020;

* A recent paper exploring the potential role of plasma p-tau217 as a biomarker for AD

[31] Mattsson-Carlgren N, Janelidze S, Bateman R, et al. Soluble P-tau217 reects both amyloid and tau pathology in the human brain and mediates the association of amyloid with neocortical tau. Brain [Internet]. 2020 [cited 2020 Nov 22];143:3234-3241. Available from: https://doi.org/10.21203/rs.3.rs-101153/v1.

\section{* A recent paper exploring the potential role of p-tau217 as a biomarker for AD}

[32] Pitt JJ. Principles and applications of liquid chromatography-mass spectrometry in clinical biochemistry. Clin Biochem Rev [Internet]. 2009 [cited 2020 Nov 22];30:19-34. Available from: http://www.ncbi.nlm.nih.gov/pubmed/19224008.

[33] Barthélemy NR, Horie K, Sato C, et al. Blood plasma phosphorylated-tau isoforms track CNS change in Alzheimer's disease. J Exp Med [Internet]. 2020 [cited 2020 Nov 22];217. Available from: https://pubmed.ncbi.nlm.nih.gov/32725127/. 
[34] Chieh JJ, Yang SY, Horng HE, et al. Immunomagnetic reduction assay using high-Tc superconducting-quantum-interference-device-based magnetosusceptometry. J Appl Phys [Internet]. 2010 [cited 2020 Nov 22];107:074903. Available from: http://aip.scitation.org/doi/10.1063/1.3340861.

[35] Yang SY, Chiu MJ, Chen TF, et al. Detection of Plasma Biomarkers Using Immunomagnetic Reduction: A Promising Method for the Early Diagnosis of Alzheimer's Disease. Neurol Ther. 2017;6:37-56.

[36] Lue LF, Sabbagh MN, Chiu MJ, et al. Plasma levels of A 342 and Tau identified probable Alzheimer's dementia: Findings in two cohorts. Front Aging Neurosci [Internet]. 2017 [cited 2020 Nov 22];9. Available from: https://pubmed.ncbi.nlm.nih.gov/28790911/.

[37] Lin CH, Yang SY, Horng HE, et al. Plasma biomarkers differentiate Parkinson's disease from atypical parkinsonism syndromes. Front Aging Neurosci [Internet]. 2018 [cited 2020 Oct 16];10. Available from: https://pubmed.ncbi.nlm.nih.gov/29755341/.

[38] Fan LY, Tzen KY, Chen YF, et al. The relation between brain amyloid deposition, cortical atrophy, and plasma biomarkers in amnesic mild cognitive impairment and Alzheimer's Disease. Front Aging Neurosci [Internet]. 2018 [cited 2020 Nov 22];10. Available from: https://pubmed.ncbi.nlm.nih.gov/29967578/.

[39] Yang CC, Chiu MJ, Chen TF, et al. Assay of plasma phosphorylated tau protein (threonine 181) and total tau protein in early-stage Alzheimer's disease. J Alzheimer's Dis [Internet]. 2018 [cited 2020 Nov 22];61:1323-1332. Available from: https://pubmed.ncbi.nlm.nih.gov/29376870/.

[40] Manca M, Kraus A. Defining the protein seeds of neurodegeneration using real-time quaking-induced conversion assays. Biomolecules. 2020.

[41] Saijo E, Metrick MA, Shunsuke II, et al. 4 - Repeat tau seeds and templating subtypes as brain and CSF biomarkers of frontotemporal lobar degeneration. Acta Neuropathol [Internet]. 2020;139:63-77. Available from: https://doi.org/10.1007/s00401-019-02080-2. * A paper presenting the potential applications of RT-QuIC for the differentiation of 3R and $4 R$ tauopathies

[42] Baldacci F, Lista S, Vergallo A, et al. A frontline defense against neurodegenerative diseases:the development of early disease detection methods. Expert Rev Mol Diagn. 2019;19:559-563.

[43] D'abramo C, D'adamio L, Giliberto L. Significance of blood and cerebrospinal fluid biomarkers for alzheimer's disease: Sensitivity, specificity and potential for clinical use. J Pers Med [Internet]. 2020 [cited 2020 Nov 22];10:1-39. Available from: 
[44] Olsson B, Lautner R, Andreasson U, et al. CSF and blood biomarkers for the diagnosis of Alzheimer's disease: a systematic review and meta-analysis. Lancet Neurol [Internet]. 2016 [cited 2020 Nov 22];15:673-684. Available from: https://pubmed.ncbi.nlm.nih.gov/27068280/.

[45] Zetterberg H, Wilson D, Andreasson U, et al. Plasma tau levels in Alzheimer's disease [Internet]. Alzheimer's Res. Ther. Alzheimers Res Ther; 2013 [cited 2020 Nov 22]. Available from: https://pubmed.ncbi.nlm.nih.gov/23551972/.

[46] Dage JL, Wennberg AMV, Airey DC, et al. Levels of tau protein in plasma are associated with neurodegeneration and cognitive function in a population-based elderly cohort. Alzheimer's Dement [Internet]. 2016 [cited 2020 Nov 22];12:1226-1234. Available from: https://pubmed.ncbi.nlm.nih.gov/27436677/.

[47] Mattsson N, Zetterberg H, Janelidze S, et al. Plasma tau in Alzheimer disease. Neurology [Internet]. 2016 [cited 2020 Nov 22];87:1827-1835. Available from: https://pubmed.ncbi.nlm.nih.gov/27694257/.

[48] Müller S, Preische O, Göpfert JC, et al. Tau plasma levels in subjective cognitive decline: Results from the DELCODE study. Sci Rep [Internet]. 2017 [cited 2020 Nov 22];7. Available from: https://pubmed.ncbi.nlm.nih.gov/28842559/.

[49] Pase MP, Beiser AS, Himali JJ, et al. Assessment of Plasma Total Tau Level as a Predictive Biomarker for Dementia and Related Endophenotypes. JAMA Neurol [Internet]. 2019 [cited 2020 Nov 22];76:598-606. Available from: https://pubmed.ncbi.nlm.nih.gov/30830207/.

[50] Mielke MM, Hagen CE, Wennberg AMV, et al. Association of plasma total tau level with cognitive decline and risk of mild cognitive impairment or dementia in the Mayo Clinic study on aging. JAMA Neurol [Internet]. 2017 [cited 2020 Nov 22];74:1073-1080. Available from: https://pubmed.ncbi.nlm.nih.gov/28692710/.

[51] Cavedo E, Lista S, Houot M, et al. Plasma tau correlates with basal forebrain atrophy rates in people at risk for alzheimer disease. Neurology [Internet]. 2020 [cited 2020 Nov 22];94:e30e41. Available from: https://pubmed.ncbi.nlm.nih.gov/31801830/.

[52] Chen J, Wojta K, Yokoyama JS, et al. Genome-wide association study identifies MAPT locus influencing human plasma tau levels. 2017;

[53] Quinn JP, Corbett NJ, Kellett KAB, et al. Tau Proteolysis in the Pathogenesis of Tauopathies: Neurotoxic Fragments and Novel Biomarkers [Internet]. J. Alzheimers. Dis. NLM (Medline); 2018 [cited 2020 Nov 22]. p. 13-33. Available from: https://pubmed.ncbi.nlm.nih.gov/29630551/. 
[54] Mattsson-Carlgren N, Palmqvist S, Blennow K, et al. Increasing the reproducibility of fluid biomarker studies in neurodegenerative studies. Nat Commun [Internet]. 2020;11:1-11. Available from: http://dx.doi.org/10.1038/s41467-020-19957-6.

[55] Barthélemy NR, Li Y, Joseph-Mathurin N, et al. A soluble phosphorylated tau signature links tau, amyloid and the evolution of stages of dominantly inherited Alzheimer's disease. Nat Med [Internet]. 2020 [cited 2021 Jan 23];26:398-407. Available from: https://pubmed.ncbi.nlm.nih.gov/32161412/.

[56] Suárez-Calvet M, Karikari TK, Ashton NJ, et al. Novel tau biomarkers phosphorylated at T181, T217 or T231 rise in the initial stages of the preclinical Alzheimer's continuum when only subtle changes in A $\beta$ pathology are detected . EMBO Mol Med [Internet]. 2020 [cited 2021 Jan 23];12. Available from: https://pubmed.ncbi.nlm.nih.gov/33169916/.

[57] Forrest SL, Kril JJ, Halliday GM. Cellular and regional vulnerability in frontotemporal tauopathies. Acta Neuropathol [Internet]. 2019;138:705-727. Available from: https://doi.org/10.1007/s00401-019-02035-7.

[58] Armstrong MJ, Litvan I, Lang AE, et al. Criteria for the diagnosis of corticobasal degeneration. Neurology. 2013;80:496-503.

[59] Arai H, Morikawa Y, Higuchi M, et al. Cerebrospinal Fluid Tau Levels in Neurodegenerative Diseases with Distinct Tau-Related Pathology. 1997;264:262-264.

[60] Schoonenboom NSM, Reesink FE, Verwey NA, et al. Cerebrospinal fluid markers for differential dementia diagnosis in a large memory clinic cohort. Neurology. 2012;78:47-54.

[61] Bech S, Hjermind LE, Salvesen L, et al. Amyloid-related biomarkers and axonal damage proteins in parkinsonian syndromes. Park Relat Disord [Internet]. 2012;18:69-72. Available from: http://dx.doi.org/10.1016/j.parkreldis.2011.08.012.

[62] Hall S, Öhrfelt A, Constantinescu R, et al. Accuracy of a panel of 5 cerebrospinal fluid biomarkers in the differential diagnosis of patients with dementia and/or Parkinsonian disorders. Arch Neurol. 2012;69:1445-1452.

[63] Magdalinou NK, Paterson RW, Schott JM, et al. A panel of nine cerebrospinal fluid biomarkers may identify patients with atypical parkinsonian syndromes. J Neurol Neurosurg Psychiatry. 2015;86:1240-1247.

[64] Schirinzi T, Maria G, Di G, et al. Clinical value of CSF amyloid-beta-42 and tau proteins in Progressive Supranuclear Palsy. J Neural Transm [Internet]. 2018;125:1373-1379. Available from: http://dx.doi.org/10.1007/s00702-018-1893-1.

[65] Jeppsson A, Wikkelsö C, Blennow K, et al. CSF biomarkers distinguish idiopathic normal pressure hydrocephalus from its mimics. 2019;1117-1123. 
[66] Urakami K, Mori M, Wada K, et al. A comparison of tau protein in cerebrospinal fluid between corticobasal degeneration and progressive supranuclear palsy. 1999;259:127-129.

[67] Urakami K, Wada K, Arai H, et al. Diagnostic significance of tau protein in cerebrospinal fluid from patients with corticobasal degeneration or progressive supranuclear palsy. 2001;183:95-98.

[68] Borroni B, Gardoni F, Parnetti L, et al. Pattern of Tau forms in CSF is altered in progressive supranuclear palsy. 2009;30:34-40.

[69] Aerts MB, Esselink RAJ, Bloem BR, et al. Cerebrospinal fluid tau and phosphorylated tau protein are elevated in corticobasal syndrome. Mov Disord. 2011;

[70] Boeve BF, Lang AE, Litvan I. Corticobasal degeneration and its relationship to progressive supranuclear palsy and frontotemporal dementia. Ann Neurol. 2003.

[71] Barthélemy NR, Gabelle A, Hirtz C, et al. Differential mass spectrometry profiles of tau protein in the cerebrospinal fluid of patients with Alzheimer's disease, progressive supranuclear palsy, and dementia with lewy bodies. J Alzheimer's Dis. 2016;51:1033-1043.

[72] Borroni B, Malinverno M, Gardoni F, Alberici A, Parnetti L, Premi E, Bonuccelli U, Grassi M, Perani D, Calabresi P, Di Luca M PA. Tau forms in CSF as a reliable biomarker for progressive supranuclear palsy. Neurology. 2008;71:1796-1803.

\section{* First report on tau fragments and truncated tau as a biomarker for PSP}

[73] Kuiperij HB, Verbeek MM. Tau forms in CSF as a reliable biomarker for progressive supranuclear palsy. Neurology. 2011;76:1443.

[74] Wagshal D, Sankaranarayanan S, Guss V, et al. Divergent CSF $\tau$ alterations in two common tauopathies: Alzheimer's disease and progressive supranuclear palsy. J Neurol Neurosurg Psychiatry. 2015;86:244-250.

[75] Karikari TK, Pascoal TA, Ashton NJ, et al. Blood phosphorylated tau 181 as a biomarker for Alzheimer's disease: a diagnostic performance and prediction modelling study using data from four prospective cohorts. Lancet Neurol [Internet]. 2020 [cited 2020 Oct 16];19:422433. Available from: https://pubmed.ncbi.nlm.nih.gov/32333900/.

[76] Constantinescu R, Axelsson M. Cerebrospinal fluid neurofilament light and tau protein as mortality biomarkers in parkinsonism. 2019;147-156.

[77] Rojas JC, Bang J, Lobach I V, et al. CSF neuro fi lament light chain and phosphorylated tau 181 predict disease progression in PSP. 2018;

[78] Bang J, Spina S, Miller BL. Frontotemporal dementia. Lancet. 2015.

[79] Irwin DJ, Grossman M, Weintraub D, et al. Neuropathological and genetic correlates of survival and dementia onset in synucleinopathies: a retrospective analysis. Lancet Neurol 
[Internet]. 2017 [cited 2020 Oct 10];16:55. Available from: /pmc/articles/PMC5181646/?report=abstract.

[80] Rascovsky K, Hodges JR, Knopman D, et al. Sensitivity of revised diagnostic criteria for the behavioural variant of frontotemporal dementia. Brain. 2011;134:2456-2477.

[81] Murley AG, Coyle-Gilchrist I, Rouse MA, et al. Redefining the multidimensional clinical phenotypes of frontotemporal lobar degeneration syndromes. Brain. 2020;143:1555-1571.

[82] Gorno-Tempini ML, Hillis AE, Weintraub S, et al. Classification of primary progressive aphasia and its variants. Neurology. 2011;76:1006-1014.

[83] Hardiman O, Al-Chalabi A, Chio A, et al. Amyotrophic lateral sclerosis. Nat Rev Dis Prim. $2017 ; 3$.

[84] van Es MA, Hardiman O, Chio A, et al. Amyotrophic lateral sclerosis. Lancet [Internet]. 2017;390:2084-2098. Available from: http://dx.doi.org/10.1016/S0140-6736(17)31287-4.

[85] Strong MJ, Abrahams S, Goldstein LH, et al. Amyotrophic lateral sclerosis - frontotemporal spectrum disorder (ALS-FTSD): Revised diagnostic criteria. Amyotroph Lateral Scler Frontotemporal Degener [Internet]. 2017/01/05. 2017;18:153-174. Available from: https://pubmed.ncbi.nlm.nih.gov/28054827.

[86] Chiò A, Moglia C, Canosa A, et al. Cognitive impairment across ALS clinical stages in a population-based cohort. Neurology. 2019;

[87] De Silva D, Hsieh S, Caga J, et al. Motor function and behaviour across the ALS-FTD spectrum. Acta Neurol Scand. 2016;

[88] Burrell JR, Kiernan MC, Vucic S, et al. Motor Neuron dysfunction in frontotemporal dementia. Brain. 2011;

[89] Bourbouli M, Rentzos M, Bougea A, et al. Cerebrospinal Fluid TAR DNA-Binding Protein 43 Combined with Tau Proteins as a Candidate Biomarker for Amyotrophic Lateral Sclerosis and Frontotemporal Dementia Spectrum Disorders. Dement Geriatr Cogn Disord. 2017;

[90] Foiani MS, Cicognola C, Ermann N, et al. Searching for novel cerebrospinal fluid biomarkers of tau pathology in frontotemporal dementia: An elusive quest. J Neurol Neurosurg Psychiatry. 2019;

** Comprehensive overview on current state of the art and limitations of CSF tau biomarkers in FTD

[91] Ye LQ, Li XY, Zhang Y Bin, et al. The discriminative capacity of CSF $\beta$-amyloid 42 and Tau in neurodegenerative diseases in the Chinese population. J Neurol Sci. 2020;

[92] Borroni B, Benussi A, Archetti S, et al. Csf p-tau181/tau ratio as biomarker for TDP pathology in frontotemporal dementia. Amyotroph Lateral Scler Front Degener. 2015; 
[93] Kämälaïnen A, Herukka SK, Hartikainen P, et al. Cerebrospinal fluid biomarkers for Alzheimer's disease in patients with frontotemporal lobar degeneration and amyotrophic lateral sclerosis with the C9ORF72 repeat expansion. Dement Geriatr Cogn Disord. 2015;

[94] Abu-Rumeileh S, Mometto N, Bartoletti-Stella A, et al. Cerebrospinal fluid biomarkers in patients with frontotemporal dementia spectrum: A single-center study. J Alzheimer's Dis. 2018;

[95] Paterson RW, Slattery CF, Poole T, et al. Cerebrospinal fluid in the differential diagnosis of Alzheimer's disease: Clinical utility of an extended panel of biomarkers in a specialist cognitive clinic. Alzheimer's Res Ther. 2018;

[96] Wilke C, Deuschle C, Rattay TW, et al. Total tau is increased, but phosphorylated tau not decreased, in cerebrospinal fluid in amyotrophic lateral sclerosis. Neurobiol Aging. 2015;

[97] Scarafino A, D'Errico E, Introna A, et al. Diagnostic and prognostic power of CSF Tau in amyotrophic lateral sclerosis. J Neurol. 2018;

[98] Abu-Rumeileh S, Vacchiano V, Zenesini C, et al. Diagnostic-prognostic value and electrophysiological correlates of CSF biomarkers of neurodegeneration and neuroinflammation in amyotrophic lateral sclerosis. J Neurol. 2020;

[99] Schreiber S, Spotorno N, Schreiber F, et al. Significance of CSF NfL and tau in ALS. J Neurol. 2018;

[100] Lanznaster D, Hergesheimer RC, Bakkouche SE, et al. A $\beta 1-42$ and tau as potential biomarkers for diagnosis and prognosis of amyotrophic lateral sclerosis. Int J Mol Sci. 2020;

[101] Vergallo A, Carlesi C, Pagni C, et al. A single center study: A $\beta 42 / p-T a u 181$ CSF ratio to discriminate AD from FTD in clinical setting. Neurol Sci. 2017;

[102] Meeter LHH, Vijverberg EG, Del Campo M, et al. Clinical value of neurofilament and phospho-tau/tau ratio in the frontotemporal dementia spectrum. Neurology. 2018;

[103] Kuiperij HB, Versleijen AAM, Beenes M, et al. Tau Rather than TDP-43 Proteins are Potential Cerebrospinal Fluid Biomarkers for Frontotemporal Lobar Degeneration Subtypes: A Pilot Study. J Alzheimer's Dis. 2017;

[104] Spillantini MG, Crowther RA, Jakes R, et al. $\alpha$-Synuclein in filamentous inclusions of Lewy bodies from Parkinson's disease and dementia with Lewy bodies. Proc Natl Acad Sci U S A [Internet]. 1998 [cited 2020 Oct 8];95:6469-6473. Available from: https://pubmed.ncbi.nlm.nih.gov/9600990/.

[105] Arima K, Hirai S, Sunohara N, et al. Cellular co-localization of phosphorylated tau- and $\mathrm{NACP} / \alpha$-synuclein- epitopes in Lewy bodies in sporadic Parkinson's disease and in dementia with Lewy bodies. Brain Res. 1999;843:53-61. 
[106] Wood SJ, Wypych J, Steavenson S, et al. $\alpha$-Synuclein fibrillogenesis is nucleationdependent: Implications for the pathogenesis of Parkinson's disease. J Biol Chem [Internet]. 1999 [cited 2020 Oct 19];274:19509-19512. Available from: https://pubmed.ncbi.nlm.nih.gov/10391881/.

[107] Friedhoff P, Von Bergen M, Mandelkow EM, et al. Structure of tau protein and assembly into paired helical filaments. Biochim. Biophys. Acta - Mol. Basis Dis. Elsevier; 2000. p. 122-132.

[108] Giasson BI, Forman MS, Higuchi M, et al. Initiation and synergistic fibrillization of tau and alpha-synuctein. Science (80- ) [Internet]. 2003 [cited 2020 Oct 16];300:636-640. Available from: https://pubmed.ncbi.nlm.nih.gov/12714745/.

[109] Souza JM, Giasson BI, Lee VMY, et al. Chaperone-like activity of synucleins. FEBS Lett [Internet]. 2000 [cited 2020 Oct 19];474:116-119. Available from: https://febs.onlinelibrary.wiley.com/doi/full/10.1016/S0014-5793\%2800\%2901563-5.

[110] Daniele S, Frosini D, Pietrobono D, et al. $\alpha$-synuclein heterocomplexes with $\beta$-amyloid are increased in red blood cells of Parkinson's disease patients and correlate with disease severity. Front Mol Neurosci [Internet]. 2018 [cited 2020 Oct 10];11. Available from: https://pubmed.ncbi.nlm.nih.gov/29520218/.

[111] Wong YC, Krainc D. $\alpha$-synuclein toxicity in neurodegeneration: Mechanism and therapeutic strategies. Nat Med [Internet]. 2017;23:1-13. Available from: http://dx.doi.org/10.1038/nm.4269.

[112] Kang JH, Irwin DJ, Chen-Plotkin AS, et al. Association of cerebrospinal fluid $\beta$-amyloid 142, t-tau, p-tau 181, and $\alpha$-synuclein levels with clinical features of drug-naive patients with early parkinson disease. JAMA Neurol [Internet]. 2013 [cited 2020 Oct 8];70:1277-1287. Available from: https://pubmed.ncbi.nlm.nih.gov/23979011/.

[113] Mollenhauer B, Caspell-Garcia CJ, Coffey CS, et al. Longitudinal CSF biomarkers in patients with early Parkinson disease and healthy controls. Neurology [Internet]. 2017 [cited 2020 Oct 8];89:1959-1969. Available from: https://pubmed.ncbi.nlm.nih.gov/29030452/.

* A paper reviewing longitudinal changes in CSF biomarkers in Parkinson's Disease

[114] Laurens B, Constantinescu R, Freeman R, et al. Fluid biomarkers in multiple system atrophy: A review of the MSA Biomarker Initiative [Internet]. Neurobiol. Dis. Academic Press Inc.; 2015 [cited 2020 Oct 8]. p. 29-41. Available from: https://pubmed.ncbi.nlm.nih.gov/25982836/.

[115] Delgado-Alvarado M, Gago B, Gorostidi A, et al. Tau/ $\alpha$-synuclein ratio and inflammatory proteins in Parkinson's disease: An exploratory study. Mov Disord [Internet]. 2017 [cited 
2020 Oct 8];32:1066-1073. Available from: https://pubmed.ncbi.nlm.nih.gov/28548309/.

[116] Aarsland D, Kurz MW. The epidemiology of dementia associated with parkinson's disease. Brain Pathol [Internet]. Brain Pathol; 2010 [cited 2020 Oct 16]. p. 633-639. Available from: https://pubmed.ncbi.nlm.nih.gov/20522088/.

[117] Parnetti L, Tiraboschi P, Lanari A, et al. Cerebrospinal Fluid Biomarkers in Parkinson's Disease with Dementia and Dementia with Lewy Bodies. Biol Psychiatry [Internet]. 2008 [cited 2020 Oct 16];64:850-855. Available from: https://pubmed.ncbi.nlm.nih.gov/18395699/.

[118] Siderowf A, Xie SX, Hurtig H, et al. CSF amyloid $\beta$ 1-42 predicts cognitive decline in Parkinson disease. Neurology [Internet]. 2010 [cited 2020 Oct 16];75:1055-1061. Available from: https://pubmed.ncbi.nlm.nih.gov/20720189/.

[119] Irwin DJ, Grossman M, Weintraub D, et al. Neuropathological and genetic correlates of survival and dementia onset in synucleinopathies: a retrospective analysis. Lancet Neurol. 2017;16:55.

[120] Mukaetova-Ladinska EB, Monteith R, Perry EK. Cerebrospinal fluid biomarkers for Dementia with Lewy Bodies [Internet]. Int. J. Alzheimers. Dis. Int J Alzheimers Dis; 2010 [cited 2020 Oct 16]. Available from: https://pubmed.ncbi.nlm.nih.gov/21048932/.

[121] Irwin DJ, Xie SX, Coughlin D, et al. CSF tau and $\beta$-amyloid predict cerebral synucleinopathy in autopsied Lewy body disorders. Neurology [Internet]. 2018 [cited 2020 Oct 10];90:e1038-e1046. Available from: https://n.neurology.org/content/90/12/e1038.

[122] Clark CM, Xie S, Chittams J, et al. Cerebrospinal Fluid Tau and $\beta$-Amyloid: How Well Do These Biomarkers Reflect Autopsy-Confirmed Dementia Diagnoses? Arch Neurol [Internet]. 2003 [cited 2020 Oct 10];60:1696-1702. Available from: https://pubmed.ncbi.nlm.nih.gov/14676043/.

[123] Parnetti L, Lanari A, Amici S, et al. CSF phosphorylated tau is a possible marker for discriminating Alzheimer's disease from dementia with Lewy bodies. Neurol Sci [Internet]. 2001 [cited 2020 Oct 10];22:77-78. Available from: https://pubmed.ncbi.nlm.nih.gov/11487210/.

[124] Schade S, Mollenhauer B. Biomarkers in biological fluids for dementia with Lewy bodies [Internet]. Alzheimer's Res. Ther. BioMed Central Ltd.; 2014 [cited 2020 Oct 10]. Available from: https://pubmed.ncbi.nlm.nih.gov/25478030/.

[125] Coughlin DG, Xie SX, Liang M, et al. Cognitive and Pathological Influences of Tau Pathology in Lewy Body Disorders Abstract HHS Public Access. Ann Neurol. 2019;85:259_ 271. 
[126] van der Zande JJ, Steenwijk MD, ten Kate M, et al. Gray matter atrophy in dementia with Lewy bodies with and without concomitant Alzheimer's disease pathology. Neurobiol Aging [Internet]. 2018 [cited 2020 Oct 16];71:171-178. Available from: https://pubmed.ncbi.nlm.nih.gov/30149288/.

[127] Abdelnour C, Ferreira D, Oppedal K, et al. The combined effect of amyloid- $\beta$ and tau biomarkers on brain atrophy in dementia with Lewy bodies. NeuroImage Clin [Internet]. 2020 [cited 2020 Oct 16];27. Available from:/pmc/articles/PMC7363702/?report=abstract.

[128] Palma JA, Norcliffe-Kaufmann L, Kaufmann H. Diagnosis of multiple system atrophy [Internet]. Auton. Neurosci. Basic Clin. Elsevier B.V.; 2018 [cited 2020 Oct 9]. p. 15-25. Available from: https://pubmed.ncbi.nlm.nih.gov/29111419/.

[129] Fanciulli A, Wenning GK. Multiple-System Atrophy. N Engl J Med. 2015;372:1375-1376.

[130] Seino Y, Nakamura T, Kawarabayashi T, et al. Cerebrospinal Fluid and Plasma Biomarkers in Neurodegenerative Diseases. J Alzheimer's Dis [Internet]. 2019 [cited 2020 Oct 10];68:395-404. Available from: https://pubmed.ncbi.nlm.nih.gov/30814356/.

[131] Herbert MK, Eeftens JM, Aerts MB, et al. CSF levels of DJ-1 and tau distinguish MSA patients from PD patients and controls. Park Relat Disord [Internet]. 2014 [cited 2020 Oct 9];20:112-115. Available from: https://pubmed.ncbi.nlm.nih.gov/24075122/.

[132] Shi M, Bradner J, Hancock AM, et al. Cerebrospinal fluid biomarkers for Parkinson disease diagnosis and progression. Ann Neurol [Internet]. 2011 [cited 2020 Oct 9];69:570-580. Available from: /pmc/articles/PMC3117674/?report=abstract.

[133] Mollenhauer B, Locascio JJ, Schulz-Schaeffer W, et al. $\alpha$-Synuclein and tau concentrations in cerebrospinal fluid of patients presenting with parkinsonism: A cohort study. Lancet Neurol. 2011;10:230-240.

[134] Constantinides VC, Paraskevas GP, Emmanouilidou E, et al. CSF biomarkers $\beta$-amyloid, tau proteins and a-synuclein in the differential diagnosis of Parkinson-plus syndromes. J Neurol Sci. 2017;382:91-95.

[135] Barbour R, Kling K, Anderson JP, et al. Red blood cells are the major source of alphasynuclein in blood. Neurodegener Dis. 2008;5:55-59.

[136] Wang X, Yu S, Li F, et al. Detection of $\alpha$-synuclein oligomers in red blood cells as a potential biomarker of Parkinson's disease. Neurosci Lett [Internet]. 2015 [cited 2020 Oct 10];599:115-119. Available from: https://pubmed.ncbi.nlm.nih.gov/25998655/.

[137] Ashton NJ, Hye A, Rajkumar AP, et al. An update on blood-based biomarkers for nonAlzheimer neurodegenerative disorders [Internet]. Nat. Rev. Neurol. Nature Research; 2020 [cited 2020 Oct 16]. p. 265-284. Available from: 
https://pubmed.ncbi.nlm.nih.gov/32322100/.

[138] Universities S, Cu M, Dubois B, et al. Advancing research diagnostic criteria for Alzheimer ' s disease : The IWG-2 criteria Position Paper Advancing research diagnostic criteria for Alzheimer' s disease : the IWG-2 criteria. Lancet Neurol. 2014;13 (6):614-649.

[139] Agdeppa ED, Kepe V, Liu J, et al. Binding characteristics of radiofluorinated 6dialkylamino-2-naphthylethylidene derivatives as positron emission tomography imaging probes for beta-amyloid plaques in Alzheimer's disease. J Neurosci. 2001;

[140] Thompson PW, Ye L, Morgenstern JL, et al. Interaction of the amyloid imaging tracer FDDNP with hallmark Alzheimer's disease pathologies. J Neurochem. 2009;

[141] Villemagne VL, Okamura N. In vivo tau imaging: Obstacles and progress. Alzheimer's Dement. 2014;

[142] Villemagne VL, Furumoto S, Fodero-Tavoletti M, et al. The challenges of tau imaging. Future Neurol. 2012.

[143] Lemoine L, Saint-Aubert L, Nennesmo I, et al. Cortical laminar tau deposits and activated astrocytes in Alzheimer's disease visualised by 3 H-THK5117 and 3 H-deprenyl autoradiography. Sci Rep. 2017;

[144] Vermeiren C, Motte P, Viot D, et al. The tau positron-emission tomography tracer AV-1451 binds with similar affinities to tau fibrils and monoamine oxidases. Mov Disord. 2018;

[145] Brosch JR, Farlow MR, Risacher SL, et al. Tau Imaging in Alzheimer's Disease Diagnosis and Clinical Trials. Neurotherapeutics. 2017.

[146] Perez-Soriano A, Arena JE, Dinelle K, et al. PBB3 imaging in Parkinsonian disorders: Evidence for binding to tau and other proteins. Mov Disord. 2017;

[147] Lois C, Gonzalez I, Johnson KA, et al. PET imaging of tau protein targets: a methodology perspective. Brain Imaging Behav. 2019;

* A comprehensive overview on tau imaging in AD

[148] Barthel H. First Tau PET Tracer Approved: Toward Accurate In Vivo Diagnosis of Alzheimer Disease. J. Nucl. Med. 2020.

[149] Braak H, Thal DR, Ghebremedhin E, et al. Stages of the Pathologic Process in Alzheimer Disease: Age Categories From 1 to 100 Years. J Neuropathol Exp Neurol. 2011;70:960-969.

[150] Tomlinson BE, Blessed G, Roth M. Observations on the brains of demented old people. J Neurol Sci. 1970;

[151] Xia CF, Arteaga J, Chen G, et al. [18F]T807, a novel tau positron emission tomography imaging agent for Alzheimer's disease. Alzheimer's Dement. 2013;

[152] Chien DT, Szardenings AK, Bahri S, et al. Early clinical PET imaging results with the novel 
PHF-tau radioligand [F18]-T808. J Alzheimer's Dis. 2014;

[153] Fleisher AS, Pontecorvo MJ, Devous MD, et al. Positron Emission Tomography Imaging with [18F]flortaucipir and Postmortem Assessment of Alzheimer Disease Neuropathologic Changes. JAMA Neurol. 2020;

[154] Ossenkoppele R, Rabinovici GD, Smith R, et al. Discriminative accuracy of [18F]flortaucipir positron emission tomography for Alzheimer disease vs other neurodegenerative disorders. JAMA - J Am Med Assoc. 2018;

[155] Harada R, Okamura N, Furumoto S, et al. 18F-THK5351: A novel PET radiotracer for imaging neurofibrillary pathology in Alzheimer disease. J Nucl Med. 2016;

[156] Schöll M, Lockhart SN, Schonhaut DR, et al. PET Imaging of Tau Deposition in the Aging Human Brain. Neuron. 2016;

[157] Johnson KA, Schultz A, Betensky RA, et al. Tau positron emission tomographic imaging in aging and early Alzheimer disease. Ann Neurol. 2016;

[158] Chiotis K, Saint-Aubert L, Savitcheva I, et al. Imaging in-vivo tau pathology in Alzheimer's disease with THK5317 PET in a multimodal paradigm. Eur J Nucl Med Mol Imaging. 2016;

[159] Cho H, Choi JY, Hwang MS, et al. In vivo cortical spreading pattern of tau and amyloid in the Alzheimer disease spectrum. Ann Neurol. 2016;

[160] Cho H, Choi JY, Hwang MS, et al. Tau PET in Alzheimer disease and mild cognitive impairment. Neurology. 2016;

[161] Pontecorvo MJ, Devous MD, Navitsky M, et al. Relationships between flortaucipir PET tau binding and amyloid burden, clinical diagnosis, age and cognition. Brain. 2017;

[162] Marshall GA, Fairbanks LA, Tekin S, et al. Early-onset Alzheimer's disease is associated with greater pathologic burden. J Geriatr Psychiatry Neurol. 2007;

[163] Sperling R, Mormino E, Johnson K. The evolution of preclinical Alzheimer's disease: Implications for prevention trials. Neuron. 2014.

[164] Ishiki A, Okamura N, Furukawa K, et al. Longitudinal assessment of Tau pathology in patients with Alzheimer's disease using [18F] THK-5117 positron emission tomography. PLoS One. 2015;

[165] Jack CR, Wiste HJ, Schwarz CG, et al. Longitudinal tau PET in ageing and Alzheimer's disease. Brain. 2018;

[166] Chiotis K, Saint-Aubert L, Rodriguez-Vieitez E, et al. Longitudinal changes of tau PET imaging in relation to hypometabolism in prodromal and Alzheimer's disease dementia. Mol Psychiatry. 2018;

[167] Cho H, Choi JY, Lee HS, et al. Progressive tau accumulation in Alzheimer disease: 2-year 
follow-up study. J Nucl Med. 2019;

[168] Hansson O, Mormino EC. Is longitudinal tau PET ready for use in Alzheimer's disease clinical trials? Brain. 2018.

[169] Ossenkoppele R, Schonhaut DR, Schöll M, et al. Tau PET patterns mirror clinical and neuroanatomical variability in Alzheimer's disease. Brain. 2016;

[170] Dronse J, Fliessbach K, Bischof GN, et al. In vivo Patterns of Tau Pathology, Amyloid- $\beta$ Burden, and Neuronal Dysfunction in Clinical Variants of Alzheimer's Disease. J

Alzheimer's Dis. 2017;

[171] Ossenkoppele R, Schonhaut DR, Baker SL, et al. Tau, amyloid, and hypometabolism in a patient with posterior cortical atrophy. Ann Neurol. 2015;

[172] Phillips JS, Das SR, McMillan CT, et al. Tau PET imaging predicts cognition in atypical variants of Alzheimer's disease. Hum Brain Mapp. 2018;

[173] Okamura N, Harada R, Ishiki A, et al. The development and validation of tau PET tracers: current status and future directions. Clin. Transl. Imaging. 2018.

[174] Leuzy A, Smith R, Ossenkoppele R, et al. Diagnostic performance of RO948 F 18 tau positron emission tomography in the differentiation of alzheimer disease from other neurodegenerative disorders. JAMA Neurol. 2020;

[175] Wong DF, Comley RA, Kuwabara H, et al. Characterization of 3 novel tau radiopharmaceuticals, 11C-RO-963,11C-RO-643, and18F-RO-948, in healthy controls and in Alzheimer subjects. J Nucl Med. 2018;

[176] Smith R, Schöll M, Leuzy A, et al. Head-to-head comparison of tau positron emission tomography tracers [18F]flortaucipir and [18F]RO948. Eur J Nucl Med Mol Imaging. 2020;

[177] Stephens A, Seibyl J, Mueller A, et al. IC-P-220: CLINICAL UPDATE: 18 F-PI-2620, A NEXT GENERATION TAU PET AGENT EVALUATED IN SUBJECTS WITH ALZHEIMER'S DISEASE AND PROGRESSIVE SUPRANUCLEAR PALSY .

Alzheimer's Dement. 2018;

[178] Betthauser TJ, Cody KA, Zammit MD, et al. In vivo characterization and quantification of neurofibrillary tau PET radioligand 18 F-MK-6240 in humans from Alzheimer disease dementia to young controls. J Nucl Med. 2019;

[179] Lohith TG, Bennacef I, Vandenberghe R, et al. Brain imaging of Alzheimer dementia patients and elderly controls with 18 F-MK-6240, a PET tracer targeting neurofibrillary tangles. J Nucl Med. 2019;

[180] Pascoal TA, Shin M, Kang MS, et al. In vivo quantification of neurofibrillary tangles with [ 18 F]MK-6240. Alzheimer's Res Ther. 2018; 
[181] Williams DR, Holton JL, Strand C, et al. Pathological tau burden and distribution distinguishes progressive supranuclear palsy-parkinsonism from Richardson's syndrome. Brain. 2007;

[182] Forman MS, Zhukareva V, Bergeron C, et al. Signature tau neuropathology in gray and white matter of corticobasal degeneration. Am J Pathol. 2002;

[183] Kouri N, Murray ME, Hassan A, et al. Neuropathological features of corticobasal degeneration presenting as corticobasal syndrome or Richardson syndrome. Brain. 2011;

[184] Ling H, Kovacs GG, Vonsattel JPG, et al. Astrogliopathy predominates the earliest stage of corticobasal degeneration pathology. Brain. 2016;

[185] Saeed U, Lang AE, Masellis M. Neuroimaging Advances in Parkinson's Disease and Atypical Parkinsonian Syndromes. Front. Neurol. 2020.

[186] Ishiki A, Harada R, Okamura N, et al. Tau imaging with [18F]THK-5351 in progressive supranuclear palsy. Eur J Neurol. 2017;

[187] Smith R, Schain M, Nilsson C, et al. Increased basal ganglia binding of 18F-AV-1451 in patients with progressive supranuclear palsy. Mov Disord. 2017;

[188] Cho H, Choi JY, Hwang MS, et al. Subcortical 18F-AV-1451 binding patterns in progressive supranuclear palsy. Mov Disord. 2017;

[189] Whitwell JL, Lowe VJ, Tosakulwong N, et al. [18F]AV-1451 tau positron emission tomography in progressive supranuclear palsy. Mov Disord. 2017;

[190] Passamonti L, Rodríguez PV, Hong YT, et al. 18F-AV-1451 positron emission tomography in Alzheimer's disease and progressive supranuclear palsy. Brain. 2017;

[191] Hammes J, Bischof GN, Giehl K, et al. Elevated in vivo [18F]-AV-1451 uptake in a patient with progressive supranuclear palsy. Mov. Disord. 2017.

[192] Coakeley S, Cho SS, Koshimori Y, et al. Positron emission tomography imaging of tau pathology in progressive supranuclear palsy. J Cereb Blood Flow Metab. 2017;

[193] Kepe V, Bordelon Y, Boxer A, et al. PET imaging of neuropathology in tauopathies: Progressive supranuclear palsy. J Alzheimer's Dis. 2013;

[194] Schonhaut DR, McMillan CT, Spina S, et al. 18F-flortaucipir tau positron emission tomography distinguishes established progressive supranuclear palsy from controls and Parkinson disease: A multicenter study. Ann Neurol. 2017;

[195] Brendel M, Schönecker S, Höglinger G, et al. [18F]-THK5351 PET correlates with topology and symptom severity in progressive supranuclear palsy. Front Aging Neurosci. 2018;

[196] Hsu JL, Chen SH, Hsiao IT, et al. 18F-THK5351 PET imaging in patients with progressive supranuclear palsy: associations with core domains and diagnostic certainty. Sci Rep. 2020; 
[197] Whitwell JL, Tosakulwong N, Botha H, et al. Brain volume and flortaucipir analysis of progressive supranuclear palsy clinical variants. NeuroImage Clin. 2020;

[198] Maruyama M, Shimada H, Suhara T, et al. Imaging of tau pathology in a tauopathy mouse model and in alzheimer patients compared to normal controls. Neuron. 2013;

[199] Kikuchi A, Okamura N, Hasegawa T, et al. In vivo visualization of tau deposits in corticobasal syndrome by 18 F-THK5351 PET. Neurology. 2016;

[200] McMillan CT, Irwin DJ, Nasrallah I, et al. Multimodal evaluation demonstrates in vivo 18FAV-1451 uptake in autopsy-confirmed corticobasal degeneration. Acta Neuropathol. 2016.

[201] Ali F, Whitwell JL, Martin PR, et al. [18F] AV-1451 uptake in corticobasal syndrome: the influence of beta-amyloid and clinical presentation. J Neurol. 2018;

[202] Tsai RM, Bejanin A, Lesman-Segev O, et al. 18F-flortaucipir (AV-1451) tau PET in frontotemporal dementia syndromes. Alzheimer's Res Ther. 2019;

[203] Lowe VJ, Curran G, Fang P, et al. An autoradiographic evaluation of AV-1451 Tau PET in dementia. Acta Neuropathol Commun. 2016;

[204] Brendel M, Barthel H, Van Eimeren T, et al. Assessment of 18F-PI-2620 as a Biomarker in Progressive Supranuclear Palsy. JAMA Neurol. 2020;

[205] Kroth H, Oden F, Molette J, et al. Discovery and preclinical characterization of [18F]PI2620, a next-generation tau PET tracer for the assessment of tau pathology in Alzheimer's disease and other tauopathies. Eur J Nucl Med Mol Imaging. 2019;

[206] Molinuevo JL, Ayton S, Batrla R, et al. Current state of Alzheimer's fluid biomarkers [Internet]. Acta Neuropathol. Springer Berlin Heidelberg; 2018. Available from: https://doi.org/10.1007/s00401-018-1932-X.

[207] Hampel H, Goetzl EJ, Kapogiannis D, et al. Biomarker-drug and liquid biopsy codevelopment for disease staging and targeted therapy: Cornerstones for Alzheimer's precision medicine and pharmacology. Front Pharmacol. 2019;10:1-10.

[208] Thijssen EH, Rabinovici GD. Rapid Progress Toward Reliable Blood Tests for Alzheimer Disease. JAMA Neurol. 2020;

[209] Janelidze S, Stomrud E, Smith R, et al. Cerebrospinal fluid p-tau217 performs better than ptau181 as a biomarker of Alzheimer's disease. Nat Commun [Internet]. 2020 [cited 2020 Dec 5];11. Available from: https://pubmed.ncbi.nlm.nih.gov/32246036/.

* A recent paper exploring the potential role of p-tau217 as a biomarker for AD

[210] Janelidze S, Berron D, Smith R, et al. Associations of Plasma Phospho-Tau217 Levels with Tau Positron Emission Tomography in Early Alzheimer Disease. JAMA Neurol. 2020;

* A recent paper exploring the potential role of p-tau217 as a biomarker for AD 
[211] Mollenhauer B, Locascio JJ, Schulz-Schaeffer W, et al. $\alpha$-Synuclein and tau concentrations in cerebrospinal fluid of patients presenting with parkinsonism: A cohort study. Lancet Neurol [Internet]. 2011 [cited 2020 Oct 10];10:230-240. Available from: https://pubmed.ncbi.nlm.nih.gov/21317042/.

[212] Hampel H, Lista S, Neri C, et al. Time for the systems-level integration of aging: Resilience enhancing strategies to prevent Alzheimer's disease. Prog Neurobiol [Internet]. 2019;181:101662. Available from: https://doi.org/10.1016/j.pneurobio.2019.101662.

[213] Toschi N, Lista S, Baldacci F, et al. Biomarker-guided clustering of Alzheimer's disease clinical syndromes. Neurobiol Aging. 2019;83:42-53.

[214] Hampel H, Caraci F, Cuello AC, et al. A Path Toward Precision Medicine for Neuroinflammatory Mechanisms in Alzheimer's Disease. Front Immunol. 2020;11. 
Progress regarding the context-of-use of tau as biomarker of Alzheimer's disease and other neurodegenerative diseases

\begin{abstract}
Introduction Tau protein misfolding and accumulation in toxic species is a critical pathophysiological process of Alzheimer's Disease (AD) and other neurodegenerative disorders (NDDs). Tau biomarkers, namely cerebrospinal fluid (CSF) total-tau (t-tau), 181-phosphorylated tau (p-tau) and tau-PET tracers, have been recently embedded in the diagnostic criteria for AD. Nevertheless, the role of tau as a diagnostic and prognostic biomarker for other NDDs remains controversial.

Areas covered We performed a systematical PubMed-based review of the most recent advances in tau-related biomarkers for NDDs. We focused on papers published from 2015 to 2020 assessing the diagnostic or prognostic value of each biomarker.

Expert opinion The assessment of tau biomarkers in alternative easily accessible matrices, through the development of ultrasensitive techniques, represents the most significant perspective for ADbiomarker research. In NDDs, novel tau isoforms (e.g., p-tau217) or proteolytic fragments (e.g., Nterminal fragments) may represent candidate diagnostic and prognostic biomarkers and may help monitoring disease progression. Protein misfolding amplification assays, allowing the identification of different tau strains (e.g. 3R-vs. 4R-tau) in CSF, may constitute a breakthrough for the in vivo stratification of NDDs. Tau-PET may help tracking the spatial-temporal evolution of tau pathophysiology in $\mathrm{AD}$ but its application outside the $\mathrm{AD}$-spectrum deserves further studies.
\end{abstract}

Keywords: Alzheimer's Disease, Fluid biomarkers, Neurodegenerative diseases, Tau, Tau-PET, Ultrasensitive techniques 


\section{Article highlights}

- Tau protein misfolding and accumulation in toxic species and tangles is a critical pathophysiological process of Alzheimer's Disease (AD) and other neurodegenerative disorders (NDDs);

- Cerebrospinal fluid (CSF) total tau (t-tau) and phosphorylated tau (p-tau) are included in the current diagnostic criteria for AD; in primary tauopathies and in other NDDs (ALS-FTD spectrum and $\alpha$-synucleinopathies) the role of tau as a diagnostic and prognostic biomarker is still controversial;

- The quantification of t-tau and p-tau and of emerging tau biomarkers in plasma by using ultrasensitive techniques are gaining momentum in the AD diagnostic workup;

- Novel tau fragments and tau isoforms detectable by means of ultrasensitive methods including Protein Misfolding Cyclic Amplification (PMCA) and Real-time quaking-induced conversion (RT-QuIC) may improve the in vivo stratification of NDDs;

- Tau-PET tracers are useful tools for the in vivo tracking of the spatial-temporal evolution of tau pathophysiology in AD but further studies are needed to define their proper context-ofuse in other tauopathies. 


\section{Introduction}

Tau is a natively unfolded microtubule-associated protein largely expressed in the central nervous system[1]. This protein plays an important role in microtubule polymerization, stabilization and remodeling, and is mainly expressed in the axons, where it also participates in regulating cellular transport processes[2,3]. Tau exists in 6 isoforms generated through the alternative splicing of the exons 2, 3 and 10 of the microtubule-associated protein tau (MAPT) gene[4]. The microtubulebinding domain (MBD) is encoded by the exon 10, whose alternative splicing produces 3 isoforms, each with 3 or 4 repeats, respectively called 3-repeat (3R) and 4-repeat (4R) tau[2]. Under pathological conditions, mutations in the MAPT gene along with a hyper-phosphorylation of tau may reduce the affinity of the MBD to the microtubules[5]. Accordingly, tau free-protein concentrations increase to a tipping point after which misfolded protein intermediates self-assembly into unwanted oligomers, fibrils, and neurofibrillary tangles (NFT)[5].

The neuronal and/or glial accumulation of aberrant tau aggregates represents the pathological hallmark of a broad spectrum of neurodegenerative disorders (NDDs) called tauopathies [6]. These include the so-called primary tauopathies, a group of NDDs, in which tau aggregates, including NFT, represent the main pathological hallmark. Primary tauopathies can be classified into 3R-tauopathies, 4R-tauopathies and mixed 3R/4R tauopathies. The major clinical phenotypes belong to the spectrum of Progressive Supranuclear Palsy (PSP), Corticobasal Degeneration (CBD) and Frontotemporal Lobar Degeneration with tau deposits (FTLD-Tau) [2,6]. Secondary tauopathies are conditions resulting from a complex interplay between tau and other misfolded proteins (e.g., amyloid- $\beta$ - A $\beta$ ) or related to alternative causes (e.g., autoimmune processes). Alzheimer's Disease (AD) represents the best characterized and more intensively studied NDDs and is classified as secondary tauopathy[2,7]. More recently, other forms of secondary tauopathies have been described including chronic traumatic encephalopathy (CTE) and the autoimmune anti-IgLON5 encephalitis [2]. Tau cytoplasmic inclusions have also been reported in Frontotemporal Dementia (FTD); up to $30 \%$ of patients with the behavioral variant of frontotemporal dementia (bvFTD) and the majority (approximately 90\%) of patients with the nonfluent variant of Primary progressive aphasia (nfvPPA) show tau pathologic depositions [8]. Also in $\alpha$-synucleinopathies, where aberrant $\alpha$-synuclein aggregates underpin the neurodegenerative mechanisms, NFT are likely to cause neurodegeneration and contribute to the disease burden $[9,10]$.

Tau biomarkers are included in the diagnostic workup of AD according to the 2011 National Institute on Aging - Alzheimer's Association (NIA-AA), the 2014 International Working Group-2 (IWG-2) criteria and the AT(N) classification system [11,12]. In 2018 the AT(N) scheme embed biomarkers of amyloid- $\beta$-related pathology (A), tau-pathology (T) and other markers of 
neurodegeneration $(\mathrm{N})$ [13]. This classification includes cerebrospinal fluid (CSF) total-tau (t-tau), as biomarker of neurodegeneration $(\mathrm{N})$ and 181-phosphorylated-tau (p-tau181 or simply p-tau), as biomarker of tau pathology [13]. These biomarkers are all increased in AD individuals. The AT(N) categorization for the first time added cerebral tau- positron emission tomography (PET) as core biomarker of AD. Indeed, the implementation of tau-PET tracers represents a recent step forward in the AD diagnostic workup [13].

Currently, the ante-mortem diagnosis of non-AD NDDs mainly relies on pure clinically based criteria. In this scenario, the diagnostic and prognostic value of tau protein in the spectrum of primary tauopathies remains unsatisfactory when measured with traditional approaches (e.g. ELISA) [14]. In fact, a biomarkers-based diagnosis is still an unmet need in NDDs and several methodological challenges should be addressed to overcome this limitation. In the last years, the use of ultrasensitive assays, the discovery of alternative tau byproducts in biofluids, and the development of innovative techniques, enabling the differentiation of tau strains, have represented potential breakthroughs for the in vivo dissection of tau pathology spectrum.

The validation of biomarkers tracking neurodegenerative processes and predicting pathophysiological progression at the earliest molecular/cellular changes is an unmet need for the early diagnosis, the prognostic evaluation and for patients selection and follow-up in targetedtherapy clinical trials [15].

We aim to review the current state of the art on the use of tau protein as a biomarker for NDDs. First, we investigated recent advances of this fluid biomarker in the diagnostic and prognostic workup of AD and other NDDs. Secondly, we evaluated the contribution of cerebral PET-imaging in tracking tau pathology across the NDDs spectrum.

\section{Literature search methods}

The aim of the present work is to review the most recent advances on tau as a biomarker for neurodegenerative diseases. A systematic PubMed-based literature search was performed to select relevant papers assessing the diagnostic and/or prognostic value of plasma tau (both $\mathrm{t}$-tau, $\mathrm{p}$-tau, and other tau fragments) in $\mathrm{AD}$ and other NDDs, as evaluated through novel ultrasensitive techniques and of CSF tau in other neurodegenerative diseases. We screened the papers using the following combination of keywords:

- for plasma tau in AD and other NDDs: ("plasma" OR "blood" OR "serum") AND ("tau") AND ("Alzheimer" OR "Corticobasal degeneration" OR "Progressive Supranuclear Palsy" OR "Amyotrophic Lateral Sclerosis" OR "Frontotemporal Dementia" OR "Parkinson" OR "Multiple System Atrophy" OR "Lewy Body")); 
- for CSF tau in NDDs (AD excluded): (("CSF") AND ("tau") AND ("Corticobasal Degeneration" OR "Progressive Supranuclear Palsy" OR "Amyotrophic Lateral Sclerosis" OR "Frontotemporal Dementia" OR "Parkinson's Disease" OR "Multiple System Atrophy" Or "Dementia With Lewy Bodies")).

Only papers written in English, assessing the prognostic and/or diagnostic value of tau and published between 2015 and 2020, were included in the final analysis. A total number of 1480 and 297 articles for plasma tau and CSF tau respectively were initially screened. Fourteen papers on plasma tau and 14 on CSF tau were retained in the final analysis (Fig.2).

For each study, we analyzed the study population, the technique used for biomarkers detection, the diagnostic and/or prognostic performance of the investigated biomarker. Given the high heterogeneity of the papers included, we used the AuROC values as a concise measure of diagnostic accuracy to compare different studies. The diagnostic value of each marker to properly allocate patients to different diagnostic groups was identified when available and classified as follows: 'excellent' (area under ROC curve [AuROC] 0.90-1.00), 'good' (AuROC: 0.80-0.89), 'fair' (AuROC: 0.70-0.79), 'poor' (AuROC: 0.60-0.69), or 'fail' (i.e., no discriminatory capacity) (AuROC: 0.50-0.59) [16]. We used hazard ratios (HR) to compare the prognostic values. Possible comparisons across studies are only qualitative, and not based on statistical analysis.

\section{Fluid biomarkers tracking tau-pathology: from conventional to ultrasensitive techniques}

Fluid biomarkers represent promising tools for the in vivo tracking of early pathophysiological changes in NDDs. Their detection and quantification in different matrices and related technical issues are regarded as hot topics in biomarkers research[14].

Tau quantification in CSF represents the first and most widely validated approach for the in vivo detection of tau protein. Tau, quantified in the CSF both as t-tau and p-tau181 currently belongs to the core $\mathrm{AD}$ biomarkers, along with amyloid- $\beta_{42}\left(\mathrm{~A} \beta_{42}\right)$, and its assessment is based on traditional Enzyme-linked immunoabsorbent assay (ELISA) techniques [11-13]. Accordingly, several commercial kits are to date available for both clinical and research purposes [17]. Even if broadly used and largely validated, standard tau quantification techniques present three main limitations: 1) they are unable to effectively quantify tau in "peripheral" matrices (e.g., plasma), where its concentration is significantly low [18], 2) they fail in detecting alternative tau species (e.g., p-tau217) 3) are ineffective in differentiating tau isoforms (e.g., $3 R$ and $4 R$ tau), seeds (e.g., tau fibrils), based on their tridimensional conformation [19].

Traditional methods show unsatisfactory and suboptimal power in differentiating patients with NDDs from healthy controls (HC) when applied to biological matrices other than CSF (e.g., blood), 
where these molecules are expressed at femtomolar ranges instead of picomoles [20]. The quantification of tau in non-CSF substrates is challenging because the concentration of the analyte may not reflect primary neurodegenerative changes. Variations in tau concentrations could be due to peripheral proteolytic processes, metabolic clearance, the interaction with other proteins and modifications of the of brain blood barrier permeability[21].

Novel techniques, developed and validated in the last five years, show better sensitivity in measuring tau levels than traditional methods by detecting up to subpicomolar and even subfemtomolar concentrations. These techniques mostly address the N-terminal rather than midregion of tau. Single Molecule Array (Simoa), a fully-automated ELISA relying on antibody-coated magnetic beads, has been used to assess t-tau, p-tau [22-24] and N-terminal tau fragments [25] in blood samples of $\mathrm{AD}$ patients (Table 1). On the other hand, Electrochemiluminescence immunoassays (ECLIA) provide a quantification of t-tau [26], p-tau181 [27-29] and p-tau217 $[30,31]$ in patients with $\mathrm{AD}$, with high accuracy even in low sample volumes (Table 1). An upgrade of standard mass spectrometry (MS), combining immunoprecipitation reactions with MS [32,33], tracks different phosphorylated tau isoforms like p-tau217 and p-tau181 in plasma [33](Table 1). Also immunomagnetic reaction (IMR) - an antibody-mediated reaction generating changes in the magnetic fields that enable the quantification of the analyte [34,35] - detects t-tau [36-39] and ptau181 [37,39] concentrations with a greater sensitivity compared to traditional techniques, but with suboptimal specificity (Table 1).

However, these ultrasensitive techniques fail in discriminating different tridimensional conformations of tau (e.g., 3R vs 4R tau). Nevertheless, the selective identification of different tau seeds represents a critical issue to understand the pathophysiology of the primary tauopathies. Based also on the hypothesis of a prion-like transcellular propagation of tau, real-time quakinginduced conversion (RT-QuIC) and protein misfolded cycle amplification (PMCA) have been successfully applied in NDDs [40]. These techniques were initially developed for the detection of prion proteins in Creutzfeldt-Jakob disease. RT-QuIC uses recombinant protein substrates undergoing shaking and incubation cycles to quantify seeding activity of prions (e.g. PrP) and prion-like proteins, such as $\alpha$-synuclein ( $\alpha$-syn) and tau strains [41]. Similarly, in PMCA seeding activity of substrates derived from brain homogenates or recombinant proteins is amplified by cycles of sonification, enabling a sensitive detection and a selective amplification of specific protein seeds [40].

More extensive research is necessary for the analytical and clinical validation of these approaches by clarifying advantages and drawbacks. Finally, an accurate qualification process, mostly based on 
clinical performance, will be required to define context(s)-of-use for pharmacological trials and clinical practice.

\section{Advances in tau as a biomarker for Alzheimer's Disease: assessing novel matrices, techniques and targets}

T-tau and p-tau proteins measured in CSF are core biomarkers of neurodegeneration in AD [11,12]. The finding of alternative tau isoforms specific for the different clinical and pathological AD subtypes may represent a significant advancement, in parallel with the recent development of ultrasensitive techniques that allow the measurement of tau proteins in blood.

To overcome several technical issues including the higher dilution of tau biomarkers in blood vs. CSF [42], several antibody-driven assays have been combined with different detection systems reaching subpicomolar and even subfemtomolar limits of detection (LOD) [43]. A metanalysis indicated that plasma t-tau is increased in AD [44]. A study performed with IMR techniques showed that plasma t-tau levels discriminated AD from controls with optimal accuracy (Table 2) [36]. By contrast, other studies conducted with Simoa and IMR reported higher plasma ttau concentrations in AD or Mild Cognitive Impairment (MCI) patients compared to HC, but with consistent overlapping results $[27,36,39,45,46]$. Furthermore, plasma t-tau is not or only weakly correlated with CSF t-tau, p-tau in different studies [45,47-49]. An inverse association between ttau and cortical thickness in AD-related areas has been reported [38,46]. Plasma t-tau was associated in $\mathrm{AD}, \mathrm{MCI}$ and $\mathrm{HC}$ with a longitudinal decline of cognitive scores and cerebral 18FDGuptake at PET imaging, increased ventricular volume, and a decrease of hippocampal volume $[47,50]$. Combining plasma t-tau into ratios (e.g., with plasma amyloid- $\beta_{1-42}$ ) increases the predictive value of cerebral tau accumulation, as assessed by PET, compared to t-tau alone [22]. On the other hand, plasma t-tau did not predict the amyloid- $\beta$-status as assessed by cerebral amyloidPET [27] or CSF [26] in AD, MCI, and HC. Intriguingly baseline plasma t-tau levels seem to be associated with atrophy of the basal forebrain cholinergic system (BFCS) in subjective memory complainers, regardless of their amyloid- $\beta$-status [51], although no differences in plasma t-tau levels emerged between SCD and HC in a further study [48]. Finally, plasma t-tau levels were found to be associated with MAPT H1c haplotype in $\mathrm{AD}, \mathrm{MCI}$ and $\mathrm{HC}$, according to a genomewide association study (GWAS) [52].

Full-length (FL) tau may account only for a small part of plasma tau proteins. Different proteolytic fragments of tau have been explored in plasma with ultrasensitive techniques so far. The levels of tau N1 fragments, measured with Simoa, are higher in AD and AD-MCI subjects than HC, 
showing good to optimal accuracy in discriminating HC from AD and MCI subjects, respectively [25] (Table 2). The actual eligibility of these fragments is still a matter of debate [53].

Conversely, plasma p-tau181 seems to be a more specific AD biomarker. Plasma p-tau181 concentrations assessed by Simoa, IMR and ECLIA-based techniques are higher in AD and MCIAD compared to HCs [23,27-29,39]. Plasma p-tau181 distinguishes AD patients from non-AD dementias taken together [28] and from vascular dementia (VaD) with optimal accuracy [23], from FTD with good to optimal accuracy $[23,29]$ and from PSP, CBD and Parkinson's disease (PD) or Multiple System Atrophy (MSA) with good accuracy [23] (Table 2). Furthermore, plasma p-tau concentration is consistent with CSF p-tau levels in A $\beta$-PET positive individuals [28,29]. Plasma ptau181 predicts the A $\beta$-PET status with good accuracy and is associated with both A $\beta$ and tau PET positivity in $\mathrm{MCI}, \mathrm{AD}$, and $\mathrm{HC}$ [27] (Table 2). Moreover, in a cohort of $\mathrm{AD}, \mathrm{MCI}$ and $\mathrm{HC}$, plasma p-tau181 correlated with tau deposition within the brain [22].

Tau isoforms other than p-tau181 have been recently studied and showed a potential role in the differential diagnosis of AD from MCI and HC. Plasma p-tau217 was able to differentiate AD (clinically assessed) from non-AD patients and autopsy-confirmed AD from non-AD patients with slightly suboptimal accuracy [30] (Table 2). Plasma p-tau-217 levels predict the amyloid- $\beta$-status as assessed by amyloid-PET or CSF A $\beta 42 / \mathrm{A} \beta 40$ ratio with optimal accuracy across preclinical-AD, AD-MCI, moderate AD, non-AD-MCI and HC [33] (Table 2). Furthermore p-tau217 could discriminate amyloid- $\beta$ positive and tau-PET negative participants from $\mathrm{HC}$ and has a potential role as an early, preclinical biomarker, being able to assess tau increase before detectable tau aggregation. Both plasma p-tau217 and p-tau181 correlated with their CSF counterparts and were shown to be released from CNS rather than from peripheral sources, thus probably representing more reliable AD biomarkers than plasma t-tau and p-tau202 [33]. Moreover, the cerebral A $\beta$ plaques and tau tangles accumulation is independently associated with ante-mortem concentrations of plasma p-tau217 in an autopsy-confirmed cohort [54]

Recently, dynamic trajectories of subsequent site-specific phosphorylation of tau have been reported from preclinical to full-blown AD stages in CSF. While p-tau217 and p-tau181 increase as early as two decades before and tend to decline close to the symptomatic stage, the levels of t-tau and p-tau205 increase later (between 17-13 estimated years to symptom onset) and constantly increase along disease progression [55] Also p-tau231 assessed in CSF by using a mid-region target antibody increases in preclinical AD, largely before p-tau 181 and p-tau217, when only subtle A $\beta$ pathology is detectable [56]. These preliminary data suggest the potential role of alternative sitespecific phosphorylated isoforms of CSF p-tau for the identification of preclinical AD and for the subsequent risk of phenoconversion. Dynamic changes of tau phosphorylation profile may also represent a potential useful biomarker to assess the efficacy of disease modifying therapies in clinical trials but a better understanding of these changes and a more extensive validation of these biomarkers and their potential context-of-use is needed. Furthermore, whether these changes are reflected in plasma and their timing is an issue that should be assessed. 


\section{The diagnostic and prognostic role of tau as a biomarker in 4R-tauopathies}

Tauopathies are pathologically characterized by a predominant intracellular accumulation of hyperphosphorylated tau fibrils [1,2] Primary tauopathies can be divided into 3R-tauopathies, including Pick's Disease (PiD), and 4R-tauopathies like PSP, CBD, Argyrophilic Grain Disease (AGD), Globular Glial Tauopathy (GGT). Mixed 3R- and 4R-tauopathies include Primary AgeRelated Tauopathy (PART) and neurofibrillary tangles dementia[2]. The genetic FTLD due to MAPT mutations may be related either to 3R-, 4R- or 3/4 R-tau pathological patterns [3](Figure 1). The clinical phenotype of these forms is highly heterogeneous and may not reflect the underlying pathology [14,57].

Currently, the diagnosis of $4 \mathrm{R}$-tauopathies relies on the post-mortem detection of diseasespecific patterns of abnormal tau aggregates [3]. Nevertheless, the in vivo diagnosis of these disorders is based on purely clinical-based criteria without reliable biomarkers to track the different tau pathologies $[3,14,58]$.

The potential role of CSF tau concentration as a diagnostic and prognostic biomarker in 4Rtauopathies (especially PSP and corticobasal syndrome - CBS) has been investigated by several studies since 1997, with conflicting results. The measurement of CSF t-tau and p-tau concentrations is helpful in the diagnostic workup of AD. Indeed the characteristic increase of tau species (t-tau or p-tau) in the CSF is specific to AD pathology and lacks in non-AD dementias (including PSP and CBS) $[59,60]$. Further, CSF t-tau and p-tau do not distinguish PD from atypical parkinsonism with enough accuracy (in particular from PSP) [61]. Nevertheless these biomarkers when combined with others in CSF - $\alpha$-synuclein, A $\beta 42$, neurofilament (NFL) [62], soluble amyloid precursor protein $\alpha$ (sAPP $\alpha$ ), soluble amyloid precursor protein $\beta$ (sAPP $\beta$ ), monocyte chemoattractant protein-1 (MCP1), YKL-40 [63] - may discriminate PSP from PD individuals, as well as CBS from PD and AD/FTD though with conflicting results (Table 3). Similarly, p-tau and t-tau CSF concentrations do not differentiate idiopathic normal pressure hydrocephalus (iNPH), a well-known parkinsonian syndrome-mimic, from PSP [64] (Table 3). Nonetheless, the combination of t-tau, A 340 and MCP1 differentiate iNPH from other neurodegenerative movement disorders taken as a whole (e.g. PD, MSA, PSP and CBD irrespective of their underlying pathology) with good accuracy (AuROC: 0.80) [65].

Within the tau-spectrum, a trend towards higher CSF tau (both t-tau and p-tau) levels has been reported in CBS compared to PSP and HCs [66-68]. Accordingly, Aerts and colleagues showed that $\mathrm{t}$-tau and $\mathrm{p}$-tau differentiated CBD from PSP with fair diagnostic accuracy (AuROC: 0.77 and 0.76 , respectively) and that increased tau levels were associated with lower Mini Mental State Examination (MMSE) scores [69]. These data suggest a role of tau biomarkers in the 
differential diagnosis of tauopathies and in the stratification of different phenotypes (e.g. motordominant vs. cognitive-dominant). However, these results should be taken with caution since ADpathology underpins CBS in about $20 \%$ of cases, thus representing a potential bias for the correct interpretation of CSF tau levels. Additionally, these data come from small samples, and patients' selection are based on obsolete diagnostic criteria $[58,70]$.

Tau isoforms may help in differentiating tauopathies from other neuropathologies and to stratify the tau-spectrum. The quantification of alternative tau fragments compared to the classic ones detected by standard ELISA methods is promising [71]. Borroni and colleagues, showed that $33 \mathrm{kDa}-\mathrm{tau} / 55 \mathrm{kDa}$-tau ratio discriminate PSP from HCs (AuROC: 0.90) and other NDDs (e.g. AD, CBD, FTD etc.) with good to excellent diagnostic accuracy (AuROC: 0.93 for AD, AuROC: 0.87 for CBD, AuROC: 0.86 for FTD) [68,72]. These authors reported a lower 33kDa-tau/55kDa-tau ratio, as assessed by semiquantitative immunoprecipitation, in patients with PSP compared to other tauopathies and $\alpha$-synucleinopathies. They also showed a correlation of this ratio with brainstem atrophy and motor impairment in PSP $[68,72]$. Other groups did not replicate these results and further investigations are needed [73].

Available commercial kits currently quantify tau concentration targeting its central core region. More recently, alternative tau fragments detected using antibodies recognizing the N-terminal fragment $\tau 12-\mathrm{BT} 2$ has been assessed, showing an excellent diagnostic accuracy in differentiating PSP from AD [74](Table 3). Furthermore, a mass spectrometry-based study (see table 1), identified 18 different CSF tau fragments with divergent patterns of expression in PSP compared to AD and to HCs [71].

In last year the application of RT-QuIC and PCMA technologies may isolate and quantify different tau strains (e.g. $4 \mathrm{R}$ and $3 \mathrm{R}$ tau) in CSF, thus representing an ideal tool to stratify in vivo 3R, 4R and mixed 3R/4R tauopathies [41].

Stepping aside from CSF, a single study explored the potential discriminating value of plasma p-tau181 in tauopathies, using ultrasensitive detection techniques (Simoa). Plasma p-tau was able to differentiate PSP and CBS from AD with good diagnostic accuracy (Table 2), being associated with A $\beta 42$ pathology assessed by cerebral amyloid-PET examinations [75]. The validation of tau-biomarkers in plasma in large longitudinal cohorts using ultrasensitive techniques is warranted.

Tau biomarkers may have a prognostic context-of-use in 4R-tauopathies. Constantinescu and colleagues showed that t-tau CSF levels may predict mortality in a cohort of PSP patients (see table 3 for the Hazard Ratio) [76]. Increased CSF t-tau levels at baseline are associated with a faster decline as measured by clinical progression scores like the Schwab and England ADL (SEADL); 
decreased baseline p-tau predicts low SEADL scores and rapid decrease in PSP Rating scale (PSPRS). The prognostic value of tau is increased when combining biomarkers into ratios (e.g., baseline p-tau, t-tau and NFL concentrations) [77]. Currently, the role of tau as prognostic biomarker in 4R-tauopathies is limited and should be clarified in longitudinal studies.

\section{The diagnostic and prognostic role of tau in ALS-FTD spectrum}

Frontotemporal dementia (FTD) and Amyotrophic lateral sclerosis (ALS) are NDDs with significantly clinical and pathological overlaps.

FTD is a heterogeneous cognitive disorder, recognizing multiple clinical and pathological phenotypes [78,79], related to the degeneration of frontal and temporal lobes [78]. Three main clinical variants of FTD have been described: the behavioral variant of FTD (bvFTD) [80], primary progressive aphasia (PPA) and the motor FTD syndromes (CBS and PSP) [81]. PPA can be further divided into a semantic variant (svPPA) and in a non-fluent variant (nfvPPA) [82]; the logopenic variant of PPA (lvPPA) is conversely considered an atypical AD variant rather than a proper FTDsyndrome [78]. On the other hand, three main pathological FTD phenotypes have been described, namely FTD with TDP-43 aggregates (FTD-TDP), FTD with tau depositions (FTD-Tau) and FTD with FUS aggregates (FTD-FUS) [8]. It should be noticed that the correlation between the clinical and the pathological phenotype is not always predictable. While nfvPPA is associated in most cases with FTD-tau ( $85 \%$ of all cases) and svPPA with FTD-TDP43 pathology ( $90 \%$ of cases), bvFTD can be associated with both FTD-tau and FTD-TDP43 histological patterns [8].

ALS is progressive NDD characterized by motor symptoms related to the progressive degeneration of the upper (UMN) and lower motoneuron (LMN) [83]. ALS is strictly associated to TDP-43 pathology in about $95 \%$ of patients, alternative pathological inclusions (e.g. SOD-1 inclusions) may be reported in specific variants in a minority of cases [83].

The clinical and pathological boundaries between FTD and ALS are blurry and the current diagnostic criteria consider ALS and FTD as a complex clinical continuum and pathologic spectrum as well $[84,85]$. In fact, ALS patients show cognitive impairment in about $50 \%$ of cases, meeting current criteria for diagnosing dementia in $10-15 \%$ of cases [86], and behavioral impairment in up to $45-50 \%$ of patients baseline [87]. Conversely, FTD patients may present motor symptoms in approximately $30 \%$ of cases showing motor neuron dysfunction (12.5\% of bvFTD patients) [88].

The assessment of Tau as a biomarker in the ALS-FTD spectrum is controversial and recent studies on CSF tau showed conflicting results.

Some authors observed a higher concentration of CSF t-tau in FTD patients compared to HC, distinguishing them with a good diagnostic accuracy [89-91], (see Table 3 for AUROC 
values). On the other hand, other investigations failed in detecting any significant difference [9295]. These conflicting results may be due to the fact that FTD is a highly heterogeneous diagnostic frame, including different clinical and pathological phenotypes. Inconsistent results have been reported in ALS studies [91,93,96-100].

Regarding the differential diagnosis between FTD and AD, p/t-Tau, t-tau and its ratios with A $\beta 42$ may be of clinical interest; FTD patients show lower levels of t-tau [91,94,95,101] and higher of $\mathrm{p} / \mathrm{t}$-Tau [94] when compared to AD patients. Moreover, the ratios between t-tau and A $\beta 42$ are able to distinguish between FTD and AD patients [91,95,101].

The CSF $p$-Tau and t-Tau ratio (p/t-Tau) is more interesting for the clinical workup. p/t-Tau was observed to be significantly lower in ALS patients [89,96-99] and in FTD patients [89,90,92,102], when compared to HC. Not surprisingly, ALS showed lower levels of CSF p/t-Tau than FTD phenotypes with a likely related tauopathy. This ratio was significantly lower in FTD variants with suspected TDP-related pathology (both patients with autopsy-proven TDP-related pathology and TDP-related mutation carriers) compared to FTD phenotypes with a likely underlying tauopathy $[89,90,92,94,102,103]$ (Table 3). Low p/t-Tau may therefore represent a marker of an underpinning TDP-43 pathology. From a pathophysiological point of view this could be due to a lower tau burden in TDP-related compared to tau-related FTLD variants. On the other hand, the different proportion of patients with concomitant MND and amyloid- $\beta$ co-pathology in the two groups may contribute to these observed differences [102]. Furthermore p/t-Tau ratio seems to correlate with motor cortex thickness and seems to be associated with UMN involvement in ALS [99]. Accordingly p-tau, t-tau or their ratio may find a context-of-use in the differentiation of clinical endophenotypes within the ALS spectrum (e.g. preponderant UMN vs. LMN involvement) but further investigations are needed.

Different concentrations of CSF tau fragments other than classic tau analytes have been reported by some authors in TDP-related vs. tau-related FTLD [90]. This suggests that differential proteolytic processes may occur in different FTLD variants but this hypotesis and its implications for novel biomarkers research deserves further studies and a more extensive assessment [90].

As regards the role of CSF tau ad a prognostic biomarker, one study showed a potential prognostic value of baseline CSF t-tau in ALS, being a reduction of overall survival observed in individuals with higher CSF t-tau values (Table 3) [97]. Moreover p/t-tau ratio has been associated with survival in FTD [102] and disease progression in ALS [100].

Moving to surrogated progression biomarkers, a negative correlation between baseline CSF t-tau and the longitudinal increase of ALS Functional Rating Scale Revised (ALSFRS-R) score was described [100]. 


\section{The diagnostic and prognostic role of tau in $\alpha$-synucleinopathies}

Parkinson's disease (PD), Dementia with Lewy Bodies (DLB) and Multiple system atrophy (MSA) belong to a group of NDDs collectively called $\alpha$-synucleinopathies. They are pathologically characterized by abnormal deposition of $\alpha$-synuclein such as the ( $\alpha$-syn)-rich neuron intracytoplasmic inclusions (Lewy bodies, Lewy neuritis and glial cytoplasmic inclusions) or the oligodendrocytes cytoplasmic inclusions [104]. Nevertheless, other misfolded proteins have been recognized in degenerated neurons, including tau proteins [105] highlighting the necessity of a more complex pathophysiological model.

In contrast to tau, which seems to require cofactors, in vitro and in vivo studies demonstrated how $\alpha$-syn is able to self-polymerize [106] [107]. Several pathophysiological mechanisms have been proposed to explain the interaction between $\alpha$-syn and tau. According to different models $\alpha$-syn can act as the "amyloidogenic seed" promoting tau aggregation with subsequent interaction between the two proteins enhancing each other's fibrillization [108] or as the pathological chaperone for tau fibrillization [109]. Whatever the mechanism, oligomeric forms of $\alpha$ syn and tau co-exist in $\alpha$-synucleinopathies and influence each other and perpetuate mutual aggregation, leading to hybrid oligomers' formation [9,110,111].

Interestingly, levels of CSF $\alpha$-syn and p-tau are directly correlated in $\alpha$-synucleinopathies. This could be because the accumulation of $\alpha$-syn in PD brain could inhibit the release of p-tau in CSF through unknown mechanisms, supporting the relationship between $\alpha$-syn and tau in $\alpha$ synucleinopathies [112]. With regard to CSF, reduced levels of $\alpha$-syn, t-tau, p-tau and amyloid- $\beta$ at disease onset have been found in PD compared to HC [112][113]. Furthermore, p-tau levels and p$/ \mathrm{t}$-tau ratio increased over time after PD onset despite stable levels of $t$-tau/ $\alpha$-syn ratio. Patients exhibiting postural instability and gait impairment predominant phenotype had lower CSF p-tau and A 442 concentrations than those with tremor-dominant phenotype [112]. Male sex and SNCA polymorphism rs316181 were linked to increased levels of p-tau, while an increased t-tau/A $\beta 42$ ratio was associated with REM behavior disorder [113].

The diagnostic value of CSF tau biomarkers is higher when they are combined into ratios [112][114][115][113][115]. The p-tau/ $\alpha$-syn ratio (which is increased in PD compared to HC), showed the highest accuracy for PD diagnosis [115] (Table 3). Roughly $75 \%$ of PD patients with a disease course longer than 10 years evolve to PD with dementia (PDD) [116]. CSF t-tau has been reported to be increased in PDD compared to HC, while p-tau did not show differences in the two groups [117]. Longitudinal studies in PD patients found neither a predictive value for the subsequent development of dementia for CSF t-tau and p-tau levels measured at PD onset [118], 
nor an association with dementia severity in PDD [117]. In contrast, plasma t-tau and p-tau181 levels measured through immunomagnetic reduction (IMR) were increased in DLB compared to HC, and reduced compared to PDD [37] (Table 2).

DLB is the second most common cause of dementia after Alzheimer's disease (AD). DLB belongs to the spectrum of $\alpha$-synucleinopathies, nevertheless, a mixed neuropathology with amyloid- $\beta$ plaques and tau pathology is present in nearly $40 \%$ of patients $(\alpha-$-syn + AD) and are associated with an increased rate of dementia and a decreased survival time [119]. Although previous studies reported increased levels of t-tau in DLB compared to HC [120], these findings were not replicated in more recent studies [121]. The use of different diagnostic criteria and populations may in part explain these inconsistent results. In general, CSF t-tau and p-tau levels are lower in DLB than AD [120], suggesting that levels of p-tau in CSF could represent a valuable marker for the differential diagnosis between these two NDDs [123]. On the other hand, t-tau levels are higher in DLB in comparison to PD and PDD, supporting the hypothesis that limbic and cortical Lewy body pathology is the main and specific pathologic correlate of dementia in PD [124]. In a neuropathological study, the regional distribution of tau and amyloid- $\beta$ is different in $\alpha$-syn $+\mathrm{AD}$ patients and $\mathrm{AD}$, with a greater proportion of tau in the temporal neocortex for DLB patients and in frontal neocortex in AD. Moreover, the severity of tau burden was correlated with worse antemortem cognitive performances in DLB individuals [125]. Tau pathology has also been correlated to the pattern of brain atrophy in MRI. Indeed, increased CSF t-tau levels were associated with high global atrophy scores [126] and elevated CSF p-tau with a more selective posterior atrophy [127]. CSF ratios t-tau/A $\beta 42$ and $p$-tau/A $\beta 42$ recently proved to predict the presence of $A D$ co-pathology in DLB, with higher levels of both ratios in $\alpha$-syn+AD brains when compared to $\alpha$-syn-AD brains, while no correlation was found analyzing single biomarkers. Besides, higher CSF t-tau/A $\beta 42$ and lower CSF A $\beta 42$ levels were associated with a higher burden of neocortical $\alpha$-syn [121].

MSA is a NDD characterized by autonomic failure associated with a poorly levodoparesponsive parkinsonian syndrome or cerebellar ataxia, thus defining two main phenotypes respectively identified as MSA-P and MSA-C $[128,129]$. Differential diagnosis between PD and MSA could be challenging because of the clinical overlap in the early stages and a reliable biomarker for the differential diagnosis of MSA from PD yet represents an unmet need. Data on CSF biomarkers in MSA are conflicting. In some studies, CSF t-tau concentration was higher in MSA than PD patients or HC [130][131], whereas others reported a reduction of its level [132]. By contrast, p-tau concentration was similar in MSA and HC [130][132]. Conflicting data have been published on the p-tau/t-tau ratio, reported either higher [130][132] or lower [133][134] in MSA compared to PD. The combination of p-tau181, t-tau and DJ1 (a protein related to PD pathogenesis 
whose function is still unknown in MSA) proved high sensitivity and specificity (respectively $82 \%$ and $81 \%$ ) to discriminate MSA from PD [131], as well as the p-tau/A $\beta 42$ ratio (specificity $71 \%$, sensibility 93\%), which is significantly increased in MSA [134] (Table 3). Similarly, the AD index $(\mathrm{CSF} A \beta 40 / 42$ ratio $\times \mathrm{t}$-tau) and the ratio t-tau/ $\alpha$-syn were higher in MSA than $\mathrm{HC}$, while no significant differences were found comparing PD and HC [130]. Finally, no CSF biomarkers proved to be reliable for the differential diagnosis between MSA-C and MSA-P [114].

Fluid biomarkers for $\alpha$-synucleinopathies were recently investigated also in blood. Red blood cells (RBC) seem to be a preferential niche for misfolded proteins [135] and oligomeric $\alpha$-syn was described in RBC of patients with PD [136]. Analysis of RBC in PD patients showed increased levels of phospho-tau (p-tau) in PD compared to HC [110]. Interestingly, RBC t-tau concentration was inversely correlated to MMSE scores both in the general PD cohort and in PD drug naïve patients, suggesting a central role of tau pathology in promoting cognitive decline in PD [110]. Plasma t-tau and p-tau were higher in MSA compared to $\mathrm{HC}$ when measured through IMR [137][37], while levels of p-tau measured through Simoa did not differ between MSA and HC [24].

\section{Tau PET in Tauopathies}

In the field of neurodegenerative dementias, in vivo imaging with radioligands designed to detect each underlying proteinopathy aims to find a gold standard biological marker. Amyloid- $\beta$ imaging has been incorporated into AD diagnostic criteria since 2007 [138]. However, cerebral amyloid-PET uptake did not correlate with cognitive performance nor neurodegeneration in AD. Biomarkers of tau pathology may be closely related to neuronal injury and changes in cognition. Tau-PET will support the investigation of the spatial-temporal evolution of tau pathophysiology in AD and other NDD as well as the clinical validation of fluid biomarkers. Therefore, research effort is focusing on the development of tau-PET radiotracers in order to reveal underlying tau deposits in a broad range of tauopathies, such as AD, FTD, PSP.

A significant number of tau PET tracers have been synthesized so far, but many of those lack sufficient specificity and selectivity $[139,140]$. The main difficulties to develop these tracers are related to [141]: 1) lipophilicity, which is necessary to cross the blood-brain barrier and the cell membrane (tau is located both intra and extracellularly); by contrast, excessive lipophilicity could lead to unspecific binding; 2) affinity for tau, relevant to obtain high selectivity for tau molecule and overcoming the high $\mathrm{A} \beta$ concentrations interfering with tau-ligand binding, but requiring prolonged scanning time to reach the steady-state; 3 ) different conformations of tau aggregates and different tau isoforms [142], making it challenging to develop a unique tau radiotracer for all types of tauopathies. Based on both in vitro and in vivo results, three families of radiotracers were 
initially synthesized: 1) the THK family (THK117, its (S)-enantiomer THK5317, and THK5351; 2) 18F-AV1451, also known as T807 or Flortaucipir; 3) PBB3. The so-called first-generation tau tracers finally showed a lack of specificity, with relevant off-target binding to monoamine oxidase B (MAO-B) for the HK family and Flortaucipir [143], neuromelanin for Flortaucipir [144], and A $\beta$-plaques and $\alpha$-syn for PBB3 [145,146]. Hence, the second generation of tau PET tracers has been developed: RO948, PI2620, JNJ311, MK6240, PM-PBB3 and AM-PBB3 [147]. However, the most widely studied agent is [18F]AV-1451 (Flortaucipir). It was the first approved tau PET tracer for estimation of the density and distribution of aggregated tau neurofibrillary tangles in adults with cognitive impairment and suspected AD [148].

\subsection{Aging}

First-generation tracers demonstrated retention of uptake confined to the medial temporal lobe in normal cognitively elderly, in accordance with pathological data [149], likely reflecting an agerelated process of tau-deposition [150]. Accumulation of tau in the temporal cortex, without an associated significant $A \beta$ burden, may suggest a promoting effect of tau in mild amnestic deficits; hippocampal atrophy or a PART related pathology in such subjects.

\subsection{Alzheimer's Disease}

There is now convincing evidence that cortical tau binding at PET imaging reflects tau accumulation reported from histopathological studies in AD brains [149]. 18F-AV1451 demonstrated high affinity in vitro for paired helical filaments (PHF) of $3 R / 4 R$ tau isoforms reported in AD brains [151,152]. A recent study in 82 individuals with or without dementia showed a high concordance between the visual reading of 18F-AV1451 PET scans and staging of cortical neurofibrillary tangles, reinforcing the concept that Flortaucipir effectively reflects pathological changes of AD [153]. Similar results were replicated in vivo, with 18F-AV1451 demonstrating to accurately differentiate clinically diagnosed AD from $\mathrm{HC}$ and other NDDs [154]. Higher levels of tau tracer retention in the inferior lateral temporal region, but also in the posterior cingulate and lateral parietal regions, provided the best discrimination areas between AD patients and HC [155-158]. Other studies comparing cognitively normal subjects with MCI patients showed differences in binding restricted to medial temporal regions (parahippocampal and entorhinal cortex) $[159,160]$, as well as lateral temporal and parietal areas when examining only amyloid- $\beta$-positive MCI individuals [158]. Furthermore, some studies reported greater retention of $18 \mathrm{~F}-\mathrm{AV} 1451$ in the cortex of younger compared to older AD patients, with a cut off of 75 years-old [161], and in early-onset compared to late-onset AD patients with a cut off of 65 
years-old [160], as similarly reported in previous post-mortem studies [162]. Moreover, neocortical Flortaucipir retention was found in preclinical AD cases and rarely in amyloid- $\beta$ negative cases $[161,163]$.

The extent of tau accumulation intimately correlates with the severity of MCI due to AD and AD dementia, providing an objective index for disease severity. The few available longitudinal tau PET studies in AD demonstrated a tracer retention related to disease duration [164-167]. However, results from the largest studies, which compared cognitively unimpaired $A \beta-$ negative and $\mathrm{A} \beta$-positive individuals, $\mathrm{MCI}$ and $\mathrm{AD}$ dementia patients, are conflicting. Cho and colleagues studied 107 participants (45 A $\beta$-negative cognitively unimpaired, $7 \mathrm{~A} \beta$-positive cognitively unimpaired, $31 \mathrm{MCI}$, and $24 \mathrm{AD}$ dementia) who completed both $18 \mathrm{~F}$-flortaucipir and $18 \mathrm{~F}$ florbetaben at baseline and who were followed up for 2 years. The authors found a predominant tau accumulation in the medial and basal temporal cortices in MCI and in the lateral temporal cortices in AD dementia [167], thus supporting a progressive tau accumulation pattern in line with the Braak model [149]. Conversely, Jack and colleagues evaluated the longitudinal change in tau PET signal during a one-year follow-up in a group of 126 individuals (59 cognitively unimpaired $A \beta$-negative, 37 cognitively unimpaired $A \beta$-positive, and 30 cognitively impaired $A \beta$ positive). They demonstrated a tau accumulation in the A $\beta$-positive clinically unimpaired group in the medial temporal lobe and in the medial parietal areas, including posterior cingulate cortex. This suggests that initial accumulation of tau aggregates in $\mathrm{AD}$ may not be restricted to the medial temporal lobes as implied by Braak staging [165]. Overall these evidence proved that longitudinal tau PET may be an useful biomarker in clinical trials to monitor the effect of diseasemodifying therapies tailored to reduce tau as well as cerebral amyloid- $\beta$ plaque burden [168]. In AD increased 18F-AV1451 uptake is strongly co-localized with hypometabolic regions and has been associated with worse performance on various cognitive domains in regionally specific patterns [169]. Accordingly, 18F-AV1451 distribution may predict the clinical variants of AD suggesting on-site neurotoxicity provoked by tau aggregates in posterior cortical atrophy, logopenic variant of primary progressive aphasia, or behavioral/dysexecutive variant of AD [169172]. The main challenge for tau tracers remains the capability to detect preclinical / prodromal / early AD stages. Importantly, the sensitivity for an early diagnosis is limited by the fact that in the early AD stages (e.g. MCI) neurofibrillary tangles are only present in deep brain regions. In more advanced AD stages 18F-AV1451 discriminate mild-to-moderate AD patients from $\mathrm{HC}$ [154].

Currently, there are not conclusive studies on second-generation tau tracers in AD patients and we do not have any head-to-head comparison between them. Available studies suggest that they may 
be more useful in identifying earlier Braak stages. They also show substantially less non-specific binding or higher affinities to primary tauopathies [173]. These agents include [18F]MK-6240 (Merck \& Co), PM-PBB3 (APRINOIA Therapeutics), F18-PI-2620 (Life Sciences) (50) and RO-948 (Roche). RO-948 tracer showed similar patterns of cerebral uptake then 18F-AV1451 [174]. Moreover, RO-948 showed a greater stability, a higher retention in the medial temporal lobe and lower intracerebral "off-target" binding than Flortaucipir [175,176]. In AD subjects, PET images of F18-PI-2620 showed a significantly higher uptake than control subjects in temporal lobe, parietal and cingulate cortex. Importantly, in non-demented control subjects it showed robust initial brain uptake and fast washout from the brain, as well as no age dependency. This last finding suggests that F18-PI-2620 could improve the discrimination between nondemented control and AD subjects in elderly. Moreover, excellent test-retest variability has been demonstrated confirming the utility of F18-PI-2620 to evaluate longitudinal change of tau deposition during disease course [177]. Finally, three recently published studies have evaluated [18F]MK-6240 in vivo [178-180]. All these studies exhibited favorable kinetic and high binding levels of [18F]MK-6240 to brain regions typically affected from NFT deposition in AD subjects. [18F]MK-6240 PET images in another cohort of patients positive for cerebral amyloid- $\beta$ deposition (cognitive unimpaired/impaired elderly controls, MCI and AD patients) showed a pattern corresponding to the anatomic distribution of tau as expected in the Braak model [178].

\subsection{Non-AD tauopathies}

Tau imaging may also be relevant for other tauopathies, such as CBD and PSP. These atypical parkinsonian syndromes are characterized by abundant filamentous tau inclusions that are made of isoforms with four microtubule-binding repeats in tubular or straight filaments. In PSP and CBD abnormal aggregation of pathologically misfolded and hyperphosphorylated tau proteins mainly occur in the basal ganglia in the early stages, spreading later to multiple brain areas: brainstem, posterior frontal lobe, cerebellum and association cortices for PSP; primary motor cortex, frontal lobe, brainstem or cerebellum for CBD [181-184]. Nevertheless, a variety of overlapping syndromes are frequent in tauopathies.

To date few studies using low specific first-generation PET tracers have examined tau binding in parkinsonian disorders. They revealed distinct patterns of tracer retention in PSP and CBD, compared to controls, showing elevated PET signal in the basal ganglia and affected cortical regions, in agreement with postmortem data [185]. 
Elevated tau deposition in PSP cases was observed in the basal ganglia, thalamus, dentate nucleus of the cerebellum, and midbrain [186-192]. However, an extensive overlap and age-dependent increase in both the PSP and control groups has been reported [187]. Significant differences in 18F-AV-1451 distribution between patients with PD and PSP, with increased uptake in the globus pallidus, midbrain, and subthalamus in PSP cases, have been also described [193,194]. Interestingly, in some studies the radioligand accumulation correlated with the clinical disease severity $[189,195,196]$, but the lack of correlations between tau binding and symptom severity was observed in the other cohorts [188,194]. A recent study by Whitwell and colleagues using flortaucipir reported that the clinical heterogeneity present in PSP is mirrored by anatomical and tau burden heterogeneity within the brain [197].

Studies analyzing the feasibility of tau PET in patients with CBS showed elevated asymmetrical tracer deposition in dentate nucleus of the cerebellum, midbrain, subthalamic nucleus, globus pallidus and putamen, precentral and postcentral cortex, superior frontal and parietal lobe, fitting with the regional distribution of tau pathology $[198,199]$. Furthermore, longitudinal investigations documented a correlation between tau accumulation over time and disease progression [166,200]. However, the degree of uptake in CBS using currently available tau ligands is variable, and some patients resulted negative $[201,202]$.

Overall, studies using in vivo tau PET in PSP and CBD individuals that received a postmortem confirmation have shown conflicting results questioning the usefulness of tau imaging in early diagnosis of such conditions. Indeed, the available tracers are not specific for $4 \mathrm{R}$ tau pathology and show substantial off-target binding in the midbrain and basal ganglia to monoamine oxidase B and to neuromelanin-containing cells [192,203].

Second generation of tracers with improved off-target binding are under evaluation also in non$\mathrm{AD}$ tauopathies. Brendel and colleagues found a great potential to diagnose patients with suspected PSP using 18F-PI-2620 which proved high affinity to recombinant 4R tau fibrils and PSP brain homogenate [204,205].

\section{Discussion and perspectives}

Epidemiological projections indicate that $\mathrm{AD}$ evolving epidemic represents a global threat for healthcare systems [54]. Minimally invasive and globally accessible tests are needed to cope with this expected burdensome demand and to manage individuals in suspected preclinical/prodromal stages of NDDs [206,207]. Blood-based biomarkers represent cost-, resource- and time effective tools [208]. They hold the potential to enable large-scale biological screening of individuals who are very unlikely to have AD-related pathophysiology and would support the request of second- 
level investigations (e.g. PET imaging or CSF assessment). Blood-based biomarkers not only open up the opportunity of a multi-step diagnostic workup but can also facilitate the reengineering of drug Research \& Development (R\&D) pipelines, from subjects' enrollment, target engagement, to treatment efficacy monitoring. In Oncology, serial liquid biopsies offer clues about the evolution of cancer in individual patients across disease stages, enabling individualized genetically and biologically guided therapies [207].

The development of novel ultrasensitive measurement techniques has enabled the detection of tau-related biomarkers, facilitating a liquid biopsy-driven paradigm shift in the field of NDDs, including AD [207]. Plasma t-tau seems to have a limited utility as diagnostic marker in NDDs when assessed alone [47]. On the other hand, plasma p-tau may find a context-of-use in the differential diagnosis between $\mathrm{AD}$ and non-AD dementias, including primary tauopathies and vascular dementia [25-29,75]. The combination of tau with other putative biomarkers (e.g. NFL) into panels or ratios seems to increase the diagnostic accuracy of tau biomarkers alone [22]. Sets of multiple biomarkers may accordingly find a context-of-use in the clinical diagnostic workup of neurodegenerative dementias, in patients' selection and their follow-up for disease modifying clinical trials.

Studies investigating p-tau isoforms other than p-tau181 (e.g. p-tau217) [30,33,209], and alternative proteolytic fragments like the N1-tau [25] fragment have shown encouraging results in differentiating $\mathrm{AD}$ and $\mathrm{MCI}$ from $\mathrm{HC}$ and in predicting the amyloid $\beta$ - in preclinical $\mathrm{AD}$. Several studies, performed in AD and NDDs, indicate that plasma p-tau217 is a reliable predictor of tau pathology (as assessed through tau-PET)[210], amyloid- $\beta$-mediated tau pathophysiology, longitudinal cortical/subcortical atrophy and AD-like cognitive decline. These findings coupled with the evidence that $\mathrm{p}$-tau2 17 discriminates $\mathrm{AD}$ from non-AD conditions [30,31] explain why plasma p-tau217 will be used as exploratory marker in different COU, including patients' selection and follow-up in clinical trials. The evidence about plasma p-tau217 also support its integration in the ATN matrix for disease diagnosis, prognosis and progression monitoring in clinical practice [31].

A better understanding of time-trajectories of these alternative phosphorylation sites of p-tau is mandatory to help understand the more adequate context-of-use of each isoform from preclinical to full-blown AD.

In primary tauopathies CSF p-tau and t-tau, as assessed by traditional techniques, are not reliable biomarkers for the diagnostic workup of 4R-tauopathies [64]. These biomarkers are useful in both clinical and research settings for differentiating NDDs with an underlying AD pathology, which can mimic primary tauopathies $[59,60]$. The detection of different tau isoforms and 
fragments (e.g. $33 \mathrm{KDa}$ vs $55 \mathrm{KDa}$ isoforms) [72] and of specific tau strains (e.g. 3R and 4R tau) by means of PMCA and RT-QuIC [41] are promising tools to stratify the pathologic tau-spectrum and to identify different phenotypes. The prognostic value of tau biomarkers in 4R-tauopathies remains to be clarified.

In the ALS-FTD spectrum, CSF t-tau and p-tau may contribute in discriminating AD from FTD and in identifying patients with FTD phenotypes with an underlying AD-pathology [91]. The diagnostic value of p-tau and t-tau alone in differentiating the FTD-ALS continuum from HCs is controversial $[89,91,98]$. Nevertheless p-tau/t-tau ratio seems to be useful in FTD for the identification of patients with a TDP-43 underlying pathology [89] and may be useful in ALS to discriminate clinical endophenotypes (UMN-predominant vs. LMN-predominant variants) [99]. Baseline CSF t-tau and p-tau/t-tau ratio could represent a prognostic marker for both FTD and ALS [97] but further studies are needed to identify the proper context-of-use of these biomarkers.

In $\alpha$-synucleinopathies the co-occurrence of underlying tauopathy is commonly reported. Ttau and p-tau concentration may vary according to PD phenotypes, thus suggesting a potential role of tau in differentiating clinical phenotypes [112,113]. An association between tau burden and cognitive impairment in PDD and DLB has been postulated but data are conflicting and inconclusive [117,118]. Similarly the diagnostic value of CSF p-tau and t-tau in atypical parkinsonism (e.g. MSA and DLB) is controversial [117,121,122,124,132,211][130,131].

As regards tau-PET tracers, tau accumulation in the medial and basal temporal cortices was observed in MCI patients, thus suggesting a potential role of tau-PET tracers in the prodromal AD and a potential value of tau-PET uptake in monitoring the efficacy of disease-modifying therapies in clinical trials $[165,167]$. A possible role of tau-PET imaging in the differential diagnosis between $4 \mathrm{R}$ tauopathies is suggested by some studies highlighting a different deposition pattern of tau in PSP compared to CBS. Off-target binding in the midbrain and basal ganglia is currently limiting the use of fist generation tracers but was partly solved with the development of secondgeneration tau tracers [145]. Further evidence on the physiological and pathologic variables influencing tau-PET tracers uptake, on the off-label binding and on the comparison of different tracers is warranted to understand the appropriate context-of-use of these biomarkers.

In conclusions, biomarkers development and related research have significantly contributed to the reengineering of $\mathrm{AD}$ as a clinical-biological framework, as reflected by the ATN system, where preclinical stages of the disease are identified and potentially treated for preventive strategies, including tau-targeting approaches. It is conceivable that the same paradigm shift may take place also for NDD others than AD. Individuals with different clinical 
presentations but similar biomarkers may be grouped into biological clusters facilitating a treatment essentially based on pathophysiological mechanisms and not phenotypes [212,213], in line with the precision medicine paradigm [214].

\section{Expert opinion}

Tau, as assessed in CSF in the form of t-tau and p-tau181, is an established diagnostic biomarker for $\mathrm{AD}$. The detection of tau in alternative easily accessible matrices like plasma currently represents one of the most exciting future directions for $\mathrm{AD}$ biomarker research.

Alternative tau isoforms (e.g. p-tau217) or proteolytic fragments (e.g. N-terminal fragments) quantified in CSF and in blood may represent candidate fluid diagnostic and prognostic markers overcoming the limitations of current CSF t-tau and p-tau measurement in NDDs. In particular plasma p-tau217 could be used as exploratory marker in different drug development pipelines with distinct molecular target and for different $\mathrm{COU}$, including patient selection, and theragnostic. The evidence about plasma p-tau217 also support its integration in the ATN matrix for disease diagnosis, prognosis and progression monitoring in clinical practice.

Nevertheless, the implementation of techniques enabling the identification of different tau strains (e.g. 3R- vs. 4R-tau), enabled by RT-QuIC and PMCA, represents the most important frontier for fluid biomarker discovery in tauopathies.

The validation of biomarkers tracking tau pathology in the ALS-FTD spectrum is challenging, due to the high clinical and phenotypical heterogeneity of this spectrum. However, tau biomarkers may identify AD-related pathology, and, to a lesser extent, differentiate tau-related from TDP-43 related pathology.

Intriguingly, tau biomarkers may reveal co-pathologies in NDDs. Biomarkers tracking tau pathology in PD and atypical parkinsonism may in fact be potentially helpful for the segregation of clinical subtypes and to combine tailor disease-modifying treatments.

The development of tau-PET tracers and the overcome of first-generation tracers' limitations (especially the off-target binding and the necessity of a cyclotron) was a great step forward in the in vivo understanding of early pathophysiological changes in $\mathrm{AD}$. Further studies are needed to define the context-of-use of tau-PET tracers in AD (especially in preclinical and prodromal AD) and their potential applications outside the $\mathrm{AD}$-spectrum.

\section{Funding}

The study was not funded. Academic study without external financial support. 


\section{Financial disclosures}

The authors have no relevant affiliations or financial involvement with any organization or entity with a financial interest in or financial conflict with the subject matter or materials discussed in the manuscript. 


\section{Bibliography}

[1] Spillantini MG, Goedert M. Tau pathology and neurodegeneration. Lancet Neurol [Internet]. 2013;12:609-622. Available from: http://dx.doi.org/10.1016/S1474-4422(13)70090-5.

[2] Rösler TW, Tayaranian Marvian A, Brendel M, et al. Four-repeat tauopathies [Internet]. Prog. Neurobiol. Elsevier Ltd; 2019 [cited 2020 Nov 22]. Available from: https://pubmed.ncbi.nlm.nih.gov/31238088/.

[3] Höglinger GU, Respondek G, Kovacs GG. New classification of tauopathies. Rev Neurol (Paris). 2018;174:664-668.

[4] Goedert M, Eisenberg DS, Crowther RA. Propagation of Tau Aggregates and Neurodegeneration. Annu Rev Neurosci [Internet]. 2017 [cited 2020 Nov 22];40:189-210. Available from: https://pubmed.ncbi.nlm.nih.gov/28772101/.

[5] La-rocque S De, Moretto E, Schiavo G. Knockin' on heaven's door : Molecular mechanisms of neuronal tau uptake. 2020;1-26.

[6] Irwin DJ. Tauopathies as clinicopathological entities. Park Relat Disord [Internet]. 2016;22:S29-S33. Available from: http://dx.doi.org/10.1016/j.parkreldis.2015.09.020.

[7] Braak H, Zetterberg H, Del Tredici K, et al. Intraneuronal tau aggregation precedes diffuse plaque deposition, but amyloid- $\beta$ changes occur before increases of tau in cerebrospinal fluid. Acta Neuropathol. 2013;126:631-641.

[8] Elahi FM, Miller BL. A clinicopathological approach to the diagnosis of dementia. Nat Rev Neurol. 2017;13:457-476.

[9] Sengupta U, Guerrero-Muñoz MJ, Castillo-Carranza DL, et al. Pathological Interface between Oligomeric Alpha-Synuclein and Tau in Synucleinopathies. Biol Psychiatry [Internet]. 2015 [cited 2020 Oct 8];78:672-683. Available from: https://pubmed.ncbi.nlm.nih.gov/25676491/.

[10] Henderson MX, Sengupta M, Trojanowski JQ, et al. Alzheimer's disease tau is a prominent pathology in LRRK2 Parkinson's disease. Acta Neuropathol Commun. 2019;7:1-16.

[11] Jack CR, Albert MS, Knopman DS, et al. Introduction to the recommendations from the National Institute on Aging-Alzheimer's Association workgroups on diagnostic guidelines for Alzheimer's disease. Alzheimer's Dement [Internet]. 2011;7:257-262. Available from: http://dx.doi.org/10.1016/j.jalz.2011.03.004.

[12] Dubois B, Feldman HH, Jacova C, et al. Advancing research diagnostic criteria for Alzheimer's disease: The IWG-2 criteria. Lancet Neurol. 2014.

[13] Jack CR, Bennett DA, Blennow K, et al. NIA-AA Research Framework: Toward a biological definition of Alzheimer's disease. Alzheimer's Dement [Internet]. 2018;14:535- 
562. Available from: https://doi.org/10.1016/j.jalz.2018.02.018.

\section{** Recommandations for a biomarker-based diagnosis of AD}

[14] Baldacci F, Mazzucchi S, Della Vecchia A, et al. The path to biomarker-based diagnostic criteria for the spectrum of neurodegenerative diseases [Internet]. Expert Rev. Mol. Diagn. Taylor and Francis Ltd; 2020 [cited 2020 Oct 8].p. 421-441. Available from: https://pubmed.ncbi.nlm.nih.gov/32066283/.

[15] Baldacci F, Lista S, Vergallo A, et al. A frontline defense against neurodegenerative diseases:the development of early disease detection methods. Expert Rev Mol Diagn [Internet]. 2019;19:559-563. Available from: https://doi.org/10.1080/14737159.2019.1627202.

[16] Xia J, Broadhurst DI, Wilson M, et al. Translational biomarker discovery in clinical metabolomics: An introductory tutorial. Metabolomics. 2013;9:280-299.

[17] Kawarabayashi T, Nakamura T, Miyashita K, et al. Novel ELISAs to measure total and phosphorylated tau in cerebrospinal fluid. Neurosci Lett [Internet]. 2020;722:134826. Available from: https://doi.org/10.1016/j.neulet.2020.134826.

[18] Blennow K, Zetterberg H. Biomarkers for Alzheimer's disease: current status and prospects for the future. J. Intern. Med. 2018.

[19] Saijo E, Metrick MA, Koga S, et al. 4-Repeat tau seeds and templating subtypes as brain and CSF biomarkers of frontotemporal lobar degeneration. Acta Neuropathol [Internet]. 2020;139:63-77. Available from: https://doi.org/10.1007/s00401-019-02080-2.

[20] Hampel H, O’Bryant SE, Molinuevo JL, et al. Blood-based biomarkers for Alzheimer disease: mapping the road to the clinic. Nat. Rev. Neurol. 2018.

[21] Del Prete E, Beatino MF, Campese N, et al. Fluid Candidate Biomarkers for Alzheimer's Disease: A Precision Medicine Approach. J Pers Med [Internet]. 2020;10:221. Available from: https://www.mdpi.com/2075-4426/10/4/221.

[22] Park JC, Han SH, Yi D, et al. Plasma tau/amyloid- $\beta$ 1-42 ratio predicts brain tau deposition and neurodegeneration in Alzheimer's disease. Brain [Internet]. 2019 [cited $2020 \mathrm{Nov}$ 22];142:771-786. Available from: https://pubmed.ncbi.nlm.nih.gov/30668647/.

[23] Karikari TK, Pascoal TA, Ashton NJ, et al. Blood phosphorylated tau 181 as a biomarker for Alzheimer' $s$ disease : a diagnostic performance and prediction modelling study using data from four prospective cohorts. 2020;19.

[24] Karikari TK, Pascoal TA, Ashton NJ, et al. Blood phosphorylated tau 181 as a biomarker for Alzheimer's disease: a diagnostic performance and prediction modelling study using data from four prospective cohorts. Lancet Neurol. 2020;19:422-433. 
[25] Chen Z, Mengel D, Keshavan A, et al. Learnings about the complexity of extracellular tau aid development of a blood-based screen for Alzheimer's disease. Alzheimer's Dement [Internet]. 2019 [cited 2020 Nov 22];15:487-496. Available from: https://pubmed.ncbi.nlm.nih.gov/30419228/.

[26] Palmqvist S, Janelidze S, Stomrud E, et al. Performance of Fully Automated Plasma Assays as Screening Tests for Alzheimer Disease-Related $\beta$-Amyloid Status. JAMA Neurol [Internet]. 2019 [cited 2020 Nov 22];76:1060-1069. Available from: https://pubmed.ncbi.nlm.nih.gov/31233127/.

[27] Mielke MM, Hagen CE, Xu J, et al. Plasma phospho-tau181 increases with Alzheimer's disease clinical severity and is associated with tau- and amyloid-positron emission tomography. Alzheimer's Dement [Internet]. 2018 [cited 2020 Nov 22];14:989-997. Available from: https://pubmed.ncbi.nlm.nih.gov/29626426/.

[28] Janelidze S, Mattsson N, Palmqvist S, et al. Plasma P-tau181 in Alzheimer's disease: relationship to other biomarkers, differential diagnosis, neuropathology and longitudinal progression to Alzheimer's dementia. Nat Med [Internet]. 2020 [cited 2020 Nov 22];26:379386. Available from: https://pubmed.ncbi.nlm.nih.gov/32123385/.

[29] Thijssen EH, La Joie R, Wolf A, et al. Diagnostic value of plasma phosphorylated tau181 in Alzheimer's disease and frontotemporal lobar degeneration. Nat Med [Internet]. 2020 [cited 2020 Nov 22];26:387-397. Available from: https://pubmed.ncbi.nlm.nih.gov/32123386/.

[30] Palmqvist S, Janelidze S, Quiroz YT, et al. Discriminative Accuracy of Plasma Phosphotau217 for Alzheimer Disease vs Other Neurodegenerative Disorders. JAMA - J Am Med Assoc. 2020;

* A recent paper exploring the potential role of plasma p-tau217 as a biomarker for AD

[31] Mattsson-Carlgren N, Janelidze S, Bateman R, et al. Soluble P-tau217 reects both amyloid and tau pathology in the human brain and mediates the association of amyloid with neocortical tau. Brain [Internet]. 2020 [cited 2020 Nov 22];143:3234-3241. Available from: https://doi.org/10.21203/rs.3.rs-101153/v1.

\section{* A recent paper exploring the potential role of p-tau217 as a biomarker for AD}

[32] Pitt JJ. Principles and applications of liquid chromatography-mass spectrometry in clinical biochemistry. Clin Biochem Rev [Internet]. 2009 [cited 2020 Nov 22];30:19-34. Available from: http://www.ncbi.nlm.nih.gov/pubmed/19224008.

[33] Barthélemy NR, Horie K, Sato C, et al. Blood plasma phosphorylated-tau isoforms track CNS change in Alzheimer's disease. J Exp Med [Internet]. 2020 [cited 2020 Nov 22];217. Available from: https://pubmed.ncbi.nlm.nih.gov/32725127/. 
[34] Chieh JJ, Yang SY, Horng HE, et al. Immunomagnetic reduction assay using high-Tc superconducting-quantum-interference-device-based magnetosusceptometry. J Appl Phys [Internet]. 2010 [cited 2020 Nov 22];107:074903. Available from: http://aip.scitation.org/doi/10.1063/1.3340861.

[35] Yang SY, Chiu MJ, Chen TF, et al. Detection of Plasma Biomarkers Using Immunomagnetic Reduction: A Promising Method for the Early Diagnosis of Alzheimer's Disease. Neurol Ther. 2017;6:37-56.

[36] Lue LF, Sabbagh MN, Chiu MJ, et al. Plasma levels of A $\beta 42$ and Tau identified probable Alzheimer's dementia: Findings in two cohorts. Front Aging Neurosci [Internet]. 2017 [cited 2020 Nov 22];9. Available from: https://pubmed.ncbi.nlm.nih.gov/28790911/.

[37] Lin CH, Yang SY, Horng HE, et al. Plasma biomarkers differentiate Parkinson's disease from atypical parkinsonism syndromes. Front Aging Neurosci [Internet]. 2018 [cited 2020 Oct 16];10. Available from: https://pubmed.ncbi.nlm.nih.gov/29755341/.

[38] Fan LY, Tzen KY, Chen YF, et al. The relation between brain amyloid deposition, cortical atrophy, and plasma biomarkers in amnesic mild cognitive impairment and Alzheimer's Disease. Front Aging Neurosci [Internet]. 2018 [cited 2020 Nov 22];10. Available from: https://pubmed.ncbi.nlm.nih.gov/29967578/.

[39] Yang CC, Chiu MJ, Chen TF, et al. Assay of plasma phosphorylated tau protein (threonine 181) and total tau protein in early-stage Alzheimer's disease. J Alzheimer's Dis [Internet]. 2018 [cited 2020 Nov 22];61:1323-1332. Available from: https://pubmed.ncbi.nlm.nih.gov/29376870/.

[40] Manca M, Kraus A. Defining the protein seeds of neurodegeneration using real-time quaking-induced conversion assays. Biomolecules. 2020.

[41] Saijo E, Metrick MA, Shunsuke II, et al. 4 - Repeat tau seeds and templating subtypes as brain and CSF biomarkers of frontotemporal lobar degeneration. Acta Neuropathol [Internet]. 2020;139:63-77. Available from: https://doi.org/10.1007/s00401-019-02080-2. * A paper presenting the potential applications of RT-QuIC for the differentiation of 3R and $4 R$ tauopathies

[42] Baldacci F, Lista S, Vergallo A, et al. A frontline defense against neurodegenerative diseases:the development of early disease detection methods. Expert Rev Mol Diagn. 2019;19:559-563.

[43] D'abramo C, D'adamio L, Giliberto L. Significance of blood and cerebrospinal fluid biomarkers for alzheimer's disease: Sensitivity, specificity and potential for clinical use. J Pers Med [Internet]. 2020 [cited 2020 Nov 22];10:1-39. Available from: 
[47] Mattsson N, Zetterberg H, Janelidze S, et al. Plasma tau in Alzheimer disease. Neurology [Internet]. 2016 [cited 2020 Nov 22];87:1827-1835. Available from: https://pubmed.ncbi.nlm.nih.gov/27694257/.

[48] Müller S, Preische O, Göpfert JC, et al. Tau plasma levels in subjective cognitive decline: Results from the DELCODE study. Sci Rep [Internet]. 2017 [cited 2020 Nov 22];7. Available from: https://pubmed.ncbi.nlm.nih.gov/28842559/.

[49] Pase MP, Beiser AS, Himali JJ, et al. Assessment of Plasma Total Tau Level as a Predictive Biomarker for Dementia and Related Endophenotypes. JAMA Neurol [Internet]. 2019 [cited 2020 Nov 22];76:598-606. Available from: https://pubmed.ncbi.nlm.nih.gov/30830207/.

[50] Mielke MM, Hagen CE, Wennberg AMV, et al. Association of plasma total tau level with cognitive decline and risk of mild cognitive impairment or dementia in the Mayo Clinic study on aging. JAMA Neurol [Internet]. 2017 [cited 2020 Nov 22];74:1073-1080. Available from: https://pubmed.ncbi.nlm.nih.gov/28692710/.

[51] Cavedo E, Lista S, Houot M, et al. Plasma tau correlates with basal forebrain atrophy rates in people at risk for alzheimer disease. Neurology [Internet]. 2020 [cited 2020 Nov 22];94:e30e41. Available from: https://pubmed.ncbi.nlm.nih.gov/31801830/.

[52] Chen J, Wojta K, Yokoyama JS, et al. Genome-wide association study identifies MAPT locus influencing human plasma tau levels. 2017;

[53] Quinn JP, Corbett NJ, Kellett KAB, et al. Tau Proteolysis in the Pathogenesis of Tauopathies: Neurotoxic Fragments and Novel Biomarkers [Internet]. J. Alzheimers. Dis. NLM (Medline); 2018 [cited 2020 Nov 22]. p. 13-33. Available from: https://pubmed.ncbi.nlm.nih.gov/29630551/. 
[54] Mattsson-Carlgren N, Palmqvist S, Blennow K, et al. Increasing the reproducibility of fluid biomarker studies in neurodegenerative studies. Nat Commun [Internet]. 2020;11:1-11. Available from: http://dx.doi.org/10.1038/s41467-020-19957-6.

[55] Barthélemy NR, Li Y, Joseph-Mathurin N, et al. A soluble phosphorylated tau signature links tau, amyloid and the evolution of stages of dominantly inherited Alzheimer's disease. Nat Med [Internet]. 2020 [cited 2021 Jan 23];26:398-407. Available from: https://pubmed.ncbi.nlm.nih.gov/32161412/.

[56] Suárez-Calvet M, Karikari TK, Ashton NJ, et al. Novel tau biomarkers phosphorylated at T181, T217 or T231 rise in the initial stages of the preclinical Alzheimer's continuum when only subtle changes in A $\beta$ pathology are detected . EMBO Mol Med [Internet]. 2020 [cited 2021 Jan 23];12. Available from: https://pubmed.ncbi.nlm.nih.gov/33169916/.

[57] Forrest SL, Kril JJ, Halliday GM. Cellular and regional vulnerability in frontotemporal tauopathies. Acta Neuropathol [Internet]. 2019;138:705-727. Available from: https://doi.org/10.1007/s00401-019-02035-7.

[58] Armstrong MJ, Litvan I, Lang AE, et al. Criteria for the diagnosis of corticobasal degeneration. Neurology. 2013;80:496-503.

[59] Arai H, Morikawa Y, Higuchi M, et al. Cerebrospinal Fluid Tau Levels in Neurodegenerative Diseases with Distinct Tau-Related Pathology. 1997;264:262-264.

[60] Schoonenboom NSM, Reesink FE, Verwey NA, et al. Cerebrospinal fluid markers for differential dementia diagnosis in a large memory clinic cohort. Neurology. 2012;78:47-54.

[61] Bech S, Hjermind LE, Salvesen L, et al. Amyloid-related biomarkers and axonal damage proteins in parkinsonian syndromes. Park Relat Disord [Internet]. 2012;18:69-72. Available from: http://dx.doi.org/10.1016/j.parkreldis.2011.08.012.

[62] Hall S, Öhrfelt A, Constantinescu R, et al. Accuracy of a panel of 5 cerebrospinal fluid biomarkers in the differential diagnosis of patients with dementia and/or Parkinsonian disorders. Arch Neurol. 2012;69:1445-1452.

[63] Magdalinou NK, Paterson RW, Schott JM, et al. A panel of nine cerebrospinal fluid biomarkers may identify patients with atypical parkinsonian syndromes. J Neurol Neurosurg Psychiatry. 2015;86:1240-1247.

[64] Schirinzi T, Maria G, Di G, et al. Clinical value of CSF amyloid-beta-42 and tau proteins in Progressive Supranuclear Palsy. J Neural Transm [Internet]. 2018;125:1373-1379. Available from: http://dx.doi.org/10.1007/s00702-018-1893-1.

[65] Jeppsson A, Wikkelsö C, Blennow K, et al. CSF biomarkers distinguish idiopathic normal pressure hydrocephalus from its mimics. 2019;1117-1123. 
[66] Urakami K, Mori M, Wada K, et al. A comparison of tau protein in cerebrospinal fluid between corticobasal degeneration and progressive supranuclear palsy. 1999;259:127-129.

[67] Urakami K, Wada K, Arai H, et al. Diagnostic significance of tau protein in cerebrospinal fluid from patients with corticobasal degeneration or progressive supranuclear palsy. 2001;183:95-98.

[68] Borroni B, Gardoni F, Parnetti L, et al. Pattern of Tau forms in CSF is altered in progressive supranuclear palsy. 2009;30:34-40.

[69] Aerts MB, Esselink RAJ, Bloem BR, et al. Cerebrospinal fluid tau and phosphorylated tau protein are elevated in corticobasal syndrome. Mov Disord. 2011;

[70] Boeve BF, Lang AE, Litvan I. Corticobasal degeneration and its relationship to progressive supranuclear palsy and frontotemporal dementia. Ann Neurol. 2003.

[71] Barthélemy NR, Gabelle A, Hirtz C, et al. Differential mass spectrometry profiles of tau protein in the cerebrospinal fluid of patients with Alzheimer's disease, progressive supranuclear palsy, and dementia with lewy bodies. J Alzheimer's Dis. 2016;51:1033-1043.

[72] Borroni B, Malinverno M, Gardoni F, Alberici A, Parnetti L, Premi E, Bonuccelli U, Grassi M, Perani D, Calabresi P, Di Luca M PA. Tau forms in CSF as a reliable biomarker for progressive supranuclear palsy. Neurology. 2008;71:1796-1803.

\section{* First report on tau fragments and truncated tau as a biomarker for PSP}

[73] Kuiperij HB, Verbeek MM. Tau forms in CSF as a reliable biomarker for progressive supranuclear palsy. Neurology. 2011;76:1443.

[74] Wagshal D, Sankaranarayanan S, Guss V, et al. Divergent CSF $\tau$ alterations in two common tauopathies: Alzheimer's disease and progressive supranuclear palsy. J Neurol Neurosurg Psychiatry. 2015;86:244-250.

[75] Karikari TK, Pascoal TA, Ashton NJ, et al. Blood phosphorylated tau 181 as a biomarker for Alzheimer's disease: a diagnostic performance and prediction modelling study using data from four prospective cohorts. Lancet Neurol [Internet]. 2020 [cited 2020 Oct 16];19:422433. Available from: https://pubmed.ncbi.nlm.nih.gov/32333900/.

[76] Constantinescu R, Axelsson M. Cerebrospinal fluid neurofilament light and tau protein as mortality biomarkers in parkinsonism. 2019;147-156.

[77] Rojas JC, Bang J, Lobach I V, et al. CSF neuro fi lament light chain and phosphorylated tau 181 predict disease progression in PSP. 2018;

[78] Bang J, Spina S, Miller BL. Frontotemporal dementia. Lancet. 2015.

[79] Irwin DJ, Grossman M, Weintraub D, et al. Neuropathological and genetic correlates of survival and dementia onset in synucleinopathies: a retrospective analysis. Lancet Neurol 
[Internet]. 2017 [cited 2020 Oct 10];16:55. Available from: /pmc/articles/PMC5181646/?report=abstract.

[80] Rascovsky K, Hodges JR, Knopman D, et al. Sensitivity of revised diagnostic criteria for the behavioural variant of frontotemporal dementia. Brain. 2011;134:2456-2477.

[81] Murley AG, Coyle-Gilchrist I, Rouse MA, et al. Redefining the multidimensional clinical phenotypes of frontotemporal lobar degeneration syndromes. Brain. 2020;143:1555-1571.

[82] Gorno-Tempini ML, Hillis AE, Weintraub S, et al. Classification of primary progressive aphasia and its variants. Neurology. 2011;76:1006-1014.

[83] Hardiman O, Al-Chalabi A, Chio A, et al. Amyotrophic lateral sclerosis. Nat Rev Dis Prim. 2017;3.

[84] van Es MA, Hardiman O, Chio A, et al. Amyotrophic lateral sclerosis. Lancet [Internet]. 2017;390:2084-2098. Available from: http://dx.doi.org/10.1016/S0140-6736(17)31287-4.

[85] Strong MJ, Abrahams S, Goldstein LH, et al. Amyotrophic lateral sclerosis - frontotemporal spectrum disorder (ALS-FTSD): Revised diagnostic criteria. Amyotroph Lateral Scler Frontotemporal Degener [Internet]. 2017/01/05. 2017;18:153-174. Available from: https://pubmed.ncbi.nlm.nih.gov/28054827.

[86] Chiò A, Moglia C, Canosa A, et al. Cognitive impairment across ALS clinical stages in a population-based cohort. Neurology. 2019;

[87] De Silva D, Hsieh S, Caga J, et al. Motor function and behaviour across the ALS-FTD spectrum. Acta Neurol Scand. 2016;

[88] Burrell JR, Kiernan MC, Vucic S, et al. Motor Neuron dysfunction in frontotemporal dementia. Brain. 2011;

[89] Bourbouli M, Rentzos M, Bougea A, et al. Cerebrospinal Fluid TAR DNA-Binding Protein 43 Combined with Tau Proteins as a Candidate Biomarker for Amyotrophic Lateral Sclerosis and Frontotemporal Dementia Spectrum Disorders. Dement Geriatr Cogn Disord. 2017;

[90] Foiani MS, Cicognola C, Ermann N, et al. Searching for novel cerebrospinal fluid biomarkers of tau pathology in frontotemporal dementia: An elusive quest. J Neurol Neurosurg Psychiatry. 2019;

** Comprehensive overview on current state of the art and limitations of CSF tau biomarkers in FTD

[91] Ye LQ, Li XY, Zhang Y Bin, et al. The discriminative capacity of CSF $\beta$-amyloid 42 and Tau in neurodegenerative diseases in the Chinese population. J Neurol Sci. 2020;

[92] Borroni B, Benussi A, Archetti S, et al. Csf p-tau181/tau ratio as biomarker for TDP pathology in frontotemporal dementia. Amyotroph Lateral Scler Front Degener. 2015; 
[93] Kämälaïnen A, Herukka SK, Hartikainen P, et al. Cerebrospinal fluid biomarkers for Alzheimer's disease in patients with frontotemporal lobar degeneration and amyotrophic lateral sclerosis with the C9ORF72 repeat expansion. Dement Geriatr Cogn Disord. 2015;

[94] Abu-Rumeileh S, Mometto N, Bartoletti-Stella A, et al. Cerebrospinal fluid biomarkers in patients with frontotemporal dementia spectrum: A single-center study. J Alzheimer's Dis. 2018;

[95] Paterson RW, Slattery CF, Poole T, et al. Cerebrospinal fluid in the differential diagnosis of Alzheimer's disease: Clinical utility of an extended panel of biomarkers in a specialist cognitive clinic. Alzheimer's Res Ther. 2018;

[96] Wilke C, Deuschle C, Rattay TW, et al. Total tau is increased, but phosphorylated tau not decreased, in cerebrospinal fluid in amyotrophic lateral sclerosis. Neurobiol Aging. 2015;

[97] Scarafino A, D'Errico E, Introna A, et al. Diagnostic and prognostic power of CSF Tau in amyotrophic lateral sclerosis. J Neurol. 2018;

[98] Abu-Rumeileh S, Vacchiano V, Zenesini C, et al. Diagnostic-prognostic value and electrophysiological correlates of CSF biomarkers of neurodegeneration and neuroinflammation in amyotrophic lateral sclerosis. J Neurol. 2020;

[99] Schreiber S, Spotorno N, Schreiber F, et al. Significance of CSF NfL and tau in ALS. J Neurol. 2018;

[100] Lanznaster D, Hergesheimer RC, Bakkouche SE, et al. A $\beta 1-42$ and tau as potential biomarkers for diagnosis and prognosis of amyotrophic lateral sclerosis. Int J Mol Sci. 2020;

[101] Vergallo A, Carlesi C, Pagni C, et al. A single center study: Aß42/p-Tau181 CSF ratio to discriminate AD from FTD in clinical setting. Neurol Sci. 2017;

[102] Meeter LHH, Vijverberg EG, Del Campo M, et al. Clinical value of neurofilament and phospho-tau/tau ratio in the frontotemporal dementia spectrum. Neurology. 2018;

[103] Kuiperij HB, Versleijen AAM, Beenes M, et al. Tau Rather than TDP-43 Proteins are Potential Cerebrospinal Fluid Biomarkers for Frontotemporal Lobar Degeneration Subtypes: A Pilot Study. J Alzheimer's Dis. 2017;

[104] Spillantini MG, Crowther RA, Jakes R, et al. $\alpha$-Synuclein in filamentous inclusions of Lewy bodies from Parkinson's disease and dementia with Lewy bodies. Proc Natl Acad Sci U S A [Internet]. 1998 [cited 2020 Oct 8];95:6469-6473. Available from: https://pubmed.ncbi.nlm.nih.gov/9600990/.

[105] Arima K, Hirai S, Sunohara N, et al. Cellular co-localization of phosphorylated tau- and NACP/ $\alpha$-synuclein- epitopes in Lewy bodies in sporadic Parkinson's disease and in dementia with Lewy bodies. Brain Res. 1999;843:53-61. 
[106] Wood SJ, Wypych J, Steavenson S, et al. $\alpha$-Synuclein fibrillogenesis is nucleationdependent: Implications for the pathogenesis of Parkinson's disease. J Biol Chem [Internet]. 1999 [cited 2020 Oct 19];274:19509-19512. Available from: https://pubmed.ncbi.nlm.nih.gov/10391881/.

[107] Friedhoff P, Von Bergen M, Mandelkow EM, et al. Structure of tau protein and assembly into paired helical filaments. Biochim. Biophys. Acta - Mol. Basis Dis. Elsevier; 2000. p. 122-132.

[108] Giasson BI, Forman MS, Higuchi M, et al. Initiation and synergistic fibrillization of tau and alpha-synuctein. Science (80- ) [Internet]. 2003 [cited 2020 Oct 16];300:636-640. Available from: https://pubmed.ncbi.nlm.nih.gov/12714745/.

[109] Souza JM, Giasson BI, Lee VMY, et al. Chaperone-like activity of synucleins. FEBS Lett [Internet]. 2000 [cited 2020 Oct 19];474:116-119. Available from: https://febs.onlinelibrary.wiley.com/doi/full/10.1016/S0014-5793\%2800\%2901563-5.

[110] Daniele S, Frosini D, Pietrobono D, et al. $\alpha$-synuclein heterocomplexes with $\beta$-amyloid are increased in red blood cells of Parkinson's disease patients and correlate with disease severity. Front Mol Neurosci [Internet]. 2018 [cited 2020 Oct 10];11. Available from: https://pubmed.ncbi.nlm.nih.gov/29520218/.

[111] Wong YC, Krainc D. $\alpha$-synuclein toxicity in neurodegeneration: Mechanism and therapeutic strategies. Nat Med [Internet]. 2017;23:1-13. Available from: http://dx.doi.org/10.1038/nm.4269.

[112] Kang JH, Irwin DJ, Chen-Plotkin AS, et al. Association of cerebrospinal fluid $\beta$-amyloid 142, t-tau, p-tau 181, and $\alpha$-synuclein levels with clinical features of drug-naive patients with early parkinson disease. JAMA Neurol [Internet]. 2013 [cited 2020 Oct 8];70:1277-1287. Available from: https://pubmed.ncbi.nlm.nih.gov/23979011/.

[113] Mollenhauer B, Caspell-Garcia CJ, Coffey CS, et al. Longitudinal CSF biomarkers in patients with early Parkinson disease and healthy controls. Neurology [Internet]. 2017 [cited 2020 Oct 8];89:1959-1969. Available from: https://pubmed.ncbi.nlm.nih.gov/29030452/.

\section{* A paper reviewing longitudinal changes in CSF biomarkers in Parkinson's Disease}

[114] Laurens B, Constantinescu R, Freeman R, et al. Fluid biomarkers in multiple system atrophy: A review of the MSA Biomarker Initiative [Internet]. Neurobiol. Dis. Academic Press Inc.; 2015 [cited 2020 Oct 8]. p. 29-41. Available from: https://pubmed.ncbi.nlm.nih.gov/25982836/.

[115] Delgado-Alvarado M, Gago B, Gorostidi A, et al. Tau/ $\alpha$-synuclein ratio and inflammatory proteins in Parkinson's disease: An exploratory study. Mov Disord [Internet]. 2017 [cited 
[116] Aarsland D, Kurz MW. The epidemiology of dementia associated with parkinson's disease. Brain Pathol [Internet]. Brain Pathol; 2010 [cited 2020 Oct 16]. p. 633-639. Available from: https://pubmed.ncbi.nlm.nih.gov/20522088/.

[117] Parnetti L, Tiraboschi P, Lanari A, et al. Cerebrospinal Fluid Biomarkers in Parkinson's Disease with Dementia and Dementia with Lewy Bodies. Biol Psychiatry [Internet]. 2008 [cited 2020 Oct 16];64:850-855. Available from: https://pubmed.ncbi.nlm.nih.gov/18395699/.

[118] Siderowf A, Xie SX, Hurtig H, et al. CSF amyloid $\beta$ 1-42 predicts cognitive decline in Parkinson disease. Neurology [Internet]. 2010 [cited 2020 Oct 16];75:1055-1061. Available from: https://pubmed.ncbi.nlm.nih.gov/20720189/.

[119] Irwin DJ, Grossman M, Weintraub D, et al. Neuropathological and genetic correlates of survival and dementia onset in synucleinopathies: a retrospective analysis. Lancet Neurol. 2017;16:55.

[120] Mukaetova-Ladinska EB, Monteith R, Perry EK. Cerebrospinal fluid biomarkers for Dementia with Lewy Bodies [Internet]. Int. J. Alzheimers. Dis. Int J Alzheimers Dis; 2010 [cited 2020 Oct 16]. Available from: https://pubmed.ncbi.nlm.nih.gov/21048932/.

[121] Irwin DJ, Xie SX, Coughlin D, et al. CSF tau and $\beta$-amyloid predict cerebral synucleinopathy in autopsied Lewy body disorders. Neurology [Internet]. 2018 [cited 2020 Oct 10];90:e1038-e1046. Available from: https://n.neurology.org/content/90/12/e1038.

[122] Clark CM, Xie S, Chittams J, et al. Cerebrospinal Fluid Tau and $\beta$-Amyloid: How Well Do These Biomarkers Reflect Autopsy-Confirmed Dementia Diagnoses? Arch Neurol [Internet]. 2003 [cited 2020 Oct 10];60:1696-1702. Available from: https://pubmed.ncbi.nlm.nih.gov/14676043/.

[123] Parnetti L, Lanari A, Amici S, et al. CSF phosphorylated tau is a possible marker for discriminating Alzheimer's disease from dementia with Lewy bodies. Neurol Sci [Internet]. 2001 [cited 2020 Oct 10];22:77-78. Available from: https://pubmed.ncbi.nlm.nih.gov/11487210/.

[124] Schade S, Mollenhauer B. Biomarkers in biological fluids for dementia with Lewy bodies [Internet]. Alzheimer's Res. Ther. BioMed Central Ltd.; 2014 [cited 2020 Oct 10]. Available from: https://pubmed.ncbi.nlm.nih.gov/25478030/.

[125] Coughlin DG, Xie SX, Liang M, et al. Cognitive and Pathological Influences of Tau Pathology in Lewy Body Disorders Abstract HHS Public Access. Ann Neurol. 2019;85:259271. 
[126] van der Zande JJ, Steenwijk MD, ten Kate M, et al. Gray matter atrophy in dementia with Lewy bodies with and without concomitant Alzheimer's disease pathology. Neurobiol Aging [Internet]. 2018 [cited 2020 Oct 16];71:171-178. Available from: https://pubmed.ncbi.nlm.nih.gov/30149288/.

[127] Abdelnour C, Ferreira D, Oppedal K, et al. The combined effect of amyloid- $\beta$ and tau biomarkers on brain atrophy in dementia with Lewy bodies. NeuroImage Clin [Internet]. 2020 [cited 2020 Oct 16];27. Available from: /pmc/articles/PMC7363702/?report=abstract.

[128] Palma JA, Norcliffe-Kaufmann L, Kaufmann H. Diagnosis of multiple system atrophy [Internet]. Auton. Neurosci. Basic Clin. Elsevier B.V.; 2018 [cited 2020 Oct 9]. p. 15-25. Available from: https://pubmed.ncbi.nlm.nih.gov/29111419/.

[129] Fanciulli A, Wenning GK. Multiple-System Atrophy. N Engl J Med. 2015;372:1375-1376.

[130] Seino Y, Nakamura T, Kawarabayashi T, et al. Cerebrospinal Fluid and Plasma Biomarkers in Neurodegenerative Diseases. J Alzheimer's Dis [Internet]. 2019 [cited 2020 Oct 10];68:395-404. Available from: https://pubmed.ncbi.nlm.nih.gov/30814356/.

[131] Herbert MK, Eeftens JM, Aerts MB, et al. CSF levels of DJ-1 and tau distinguish MSA patients from PD patients and controls. Park Relat Disord [Internet]. 2014 [cited 2020 Oct 9];20:112-115. Available from: https://pubmed.ncbi.nlm.nih.gov/24075122/.

[132] Shi M, Bradner J, Hancock AM, et al. Cerebrospinal fluid biomarkers for Parkinson disease diagnosis and progression. Ann Neurol [Internet]. 2011 [cited 2020 Oct 9];69:570-580. Available from: /pmc/articles/PMC3117674/?report=abstract.

[133] Mollenhauer B, Locascio JJ, Schulz-Schaeffer W, et al. $\alpha$-Synuclein and tau concentrations in cerebrospinal fluid of patients presenting with parkinsonism: A cohort study. Lancet Neurol. 2011;10:230-240.

[134] Constantinides VC, Paraskevas GP, Emmanouilidou E, et al. CSF biomarkers $\beta$-amyloid, tau proteins and a-synuclein in the differential diagnosis of Parkinson-plus syndromes. J Neurol Sci. 2017;382:91-95.

[135] Barbour R, Kling K, Anderson JP, et al. Red blood cells are the major source of alphasynuclein in blood. Neurodegener Dis. 2008;5:55-59.

[136] Wang X, Yu S, Li F, et al. Detection of $\alpha$-synuclein oligomers in red blood cells as a potential biomarker of Parkinson's disease. Neurosci Lett [Internet]. 2015 [cited 2020 Oct 10];599:115-119. Available from: https://pubmed.ncbi.nlm.nih.gov/25998655/.

[137] Ashton NJ, Hye A, Rajkumar AP, et al. An update on blood-based biomarkers for nonAlzheimer neurodegenerative disorders [Internet]. Nat. Rev. Neurol. Nature Research; 2020 [cited 2020 Oct 16]. p. 265-284. Available from: 
[138] Universities S, Cu M, Dubois B, et al. Advancing research diagnostic criteria for Alzheimer ' s disease : The IWG-2 criteria Position Paper Advancing research diagnostic criteria for Alzheimer's disease : the IWG-2 criteria. Lancet Neurol. 2014;13 (6):614-649.

[139] Agdeppa ED, Kepe V, Liu J, et al. Binding characteristics of radiofluorinated 6dialkylamino-2-naphthylethylidene derivatives as positron emission tomography imaging probes for beta-amyloid plaques in Alzheimer's disease. J Neurosci. 2001;

[140] Thompson PW, Ye L, Morgenstern JL, et al. Interaction of the amyloid imaging tracer FDDNP with hallmark Alzheimer's disease pathologies. J Neurochem. 2009;

[141] Villemagne VL, Okamura N. In vivo tau imaging: Obstacles and progress. Alzheimer's Dement. 2014;

[142] Villemagne VL, Furumoto S, Fodero-Tavoletti M, et al. The challenges of tau imaging. Future Neurol. 2012.

[143] Lemoine L, Saint-Aubert L, Nennesmo I, et al. Cortical laminar tau deposits and activated astrocytes in Alzheimer's disease visualised by 3 H-THK5117 and 3 H-deprenyl autoradiography. Sci Rep. 2017;

[144] Vermeiren C, Motte P, Viot D, et al. The tau positron-emission tomography tracer AV-1451 binds with similar affinities to tau fibrils and monoamine oxidases. Mov Disord. 2018;

[145] Brosch JR, Farlow MR, Risacher SL, et al. Tau Imaging in Alzheimer's Disease Diagnosis and Clinical Trials. Neurotherapeutics. 2017.

[146] Perez-Soriano A, Arena JE, Dinelle K, et al. PBB3 imaging in Parkinsonian disorders: Evidence for binding to tau and other proteins. Mov Disord. 2017;

[147] Lois C, Gonzalez I, Johnson KA, et al. PET imaging of tau protein targets: a methodology perspective. Brain Imaging Behav. 2019;

* A comprehensive overview on tau imaging in AD

[148] Barthel H. First Tau PET Tracer Approved: Toward Accurate In Vivo Diagnosis of Alzheimer Disease. J. Nucl. Med. 2020.

[149] Braak H, Thal DR, Ghebremedhin E, et al. Stages of the Pathologic Process in Alzheimer Disease: Age Categories From 1 to 100 Years. J Neuropathol Exp Neurol. 2011;70:960-969.

[150] Tomlinson BE, Blessed G, Roth M. Observations on the brains of demented old people. J Neurol Sci. 1970;

[151] Xia CF, Arteaga J, Chen G, et al. [18F]T807, a novel tau positron emission tomography imaging agent for Alzheimer's disease. Alzheimer's Dement. 2013;

[152] Chien DT, Szardenings AK, Bahri S, et al. Early clinical PET imaging results with the novel 
PHF-tau radioligand [F18]-T808. J Alzheimer's Dis. 2014;

[153] Fleisher AS, Pontecorvo MJ, Devous MD, et al. Positron Emission Tomography Imaging with [18F]flortaucipir and Postmortem Assessment of Alzheimer Disease Neuropathologic Changes. JAMA Neurol. 2020;

[154] Ossenkoppele R, Rabinovici GD, Smith R, et al. Discriminative accuracy of [18F]flortaucipir positron emission tomography for Alzheimer disease vs other neurodegenerative disorders. JAMA - J Am Med Assoc. 2018;

[155] Harada R, Okamura N, Furumoto S, et al. 18F-THK5351: A novel PET radiotracer for imaging neurofibrillary pathology in Alzheimer disease. J Nucl Med. 2016;

[156] Schöll M, Lockhart SN, Schonhaut DR, et al. PET Imaging of Tau Deposition in the Aging Human Brain. Neuron. 2016;

[157] Johnson KA, Schultz A, Betensky RA, et al. Tau positron emission tomographic imaging in aging and early Alzheimer disease. Ann Neurol. 2016;

[158] Chiotis K, Saint-Aubert L, Savitcheva I, et al. Imaging in-vivo tau pathology in Alzheimer's disease with THK5317 PET in a multimodal paradigm. Eur J Nucl Med Mol Imaging. 2016;

[159] Cho H, Choi JY, Hwang MS, et al. In vivo cortical spreading pattern of tau and amyloid in the Alzheimer disease spectrum. Ann Neurol. 2016;

[160] Cho H, Choi JY, Hwang MS, et al. Tau PET in Alzheimer disease and mild cognitive impairment. Neurology. 2016;

[161] Pontecorvo MJ, Devous MD, Navitsky M, et al. Relationships between flortaucipir PET tau binding and amyloid burden, clinical diagnosis, age and cognition. Brain. 2017;

[162] Marshall GA, Fairbanks LA, Tekin S, et al. Early-onset Alzheimer's disease is associated with greater pathologic burden. J Geriatr Psychiatry Neurol. 2007;

[163] Sperling R, Mormino E, Johnson K. The evolution of preclinical Alzheimer's disease: Implications for prevention trials. Neuron. 2014.

[164] Ishiki A, Okamura N, Furukawa K, et al. Longitudinal assessment of Tau pathology in patients with Alzheimer's disease using [18F] THK-5117 positron emission tomography. PLoS One. 2015;

[165] Jack CR, Wiste HJ, Schwarz CG, et al. Longitudinal tau PET in ageing and Alzheimer's disease. Brain. 2018;

[166] Chiotis K, Saint-Aubert L, Rodriguez-Vieitez E, et al. Longitudinal changes of tau PET imaging in relation to hypometabolism in prodromal and Alzheimer's disease dementia. Mol Psychiatry. 2018;

[167] Cho H, Choi JY, Lee HS, et al. Progressive tau accumulation in Alzheimer disease: 2-year 
follow-up study. J Nucl Med. 2019;

[168] Hansson O, Mormino EC. Is longitudinal tau PET ready for use in Alzheimer's disease clinical trials? Brain. 2018.

[169] Ossenkoppele R, Schonhaut DR, Schöll M, et al. Tau PET patterns mirror clinical and neuroanatomical variability in Alzheimer's disease. Brain. 2016;

[170] Dronse J, Fliessbach K, Bischof GN, et al. In vivo Patterns of Tau Pathology, Amyloid- $\beta$ Burden, and Neuronal Dysfunction in Clinical Variants of Alzheimer's Disease. J

Alzheimer's Dis. 2017;

[171] Ossenkoppele R, Schonhaut DR, Baker SL, et al. Tau, amyloid, and hypometabolism in a patient with posterior cortical atrophy. Ann Neurol. 2015;

[172] Phillips JS, Das SR, McMillan CT, et al. Tau PET imaging predicts cognition in atypical variants of Alzheimer's disease. Hum Brain Mapp. 2018;

[173] Okamura N, Harada R, Ishiki A, et al. The development and validation of tau PET tracers: current status and future directions. Clin. Transl. Imaging. 2018.

[174] Leuzy A, Smith R, Ossenkoppele R, et al. Diagnostic performance of RO948 F 18 tau positron emission tomography in the differentiation of alzheimer disease from other neurodegenerative disorders. JAMA Neurol. 2020;

[175] Wong DF, Comley RA, Kuwabara H, et al. Characterization of 3 novel tau radiopharmaceuticals, 11C-RO-963,11C-RO-643, and18F-RO-948, in healthy controls and in Alzheimer subjects. J Nucl Med. 2018;

[176] Smith R, Schöll M, Leuzy A, et al. Head-to-head comparison of tau positron emission tomography tracers [18F]flortaucipir and [18F]RO948. Eur J Nucl Med Mol Imaging. 2020;

[177] Stephens A, Seibyl J, Mueller A, et al. IC-P-220: CLINICAL UPDATE: 18 F-PI-2620, A NEXT GENERATION TAU PET AGENT EVALUATED IN SUBJECTS WITH ALZHEIMER'S DISEASE AND PROGRESSIVE SUPRANUCLEAR PALSY .

Alzheimer's Dement. 2018;

[178] Betthauser TJ, Cody KA, Zammit MD, et al. In vivo characterization and quantification of neurofibrillary tau PET radioligand 18 F-MK-6240 in humans from Alzheimer disease dementia to young controls. J Nucl Med. 2019;

[179] Lohith TG, Bennacef I, Vandenberghe R, et al. Brain imaging of Alzheimer dementia patients and elderly controls with 18 F-MK-6240, a PET tracer targeting neurofibrillary tangles. J Nucl Med. 2019;

[180] Pascoal TA, Shin M, Kang MS, et al. In vivo quantification of neurofibrillary tangles with [ 18 F]MK-6240. Alzheimer's Res Ther. 2018; 
[181] Williams DR, Holton JL, Strand C, et al. Pathological tau burden and distribution distinguishes progressive supranuclear palsy-parkinsonism from Richardson's syndrome. Brain. 2007;

[182] Forman MS, Zhukareva V, Bergeron C, et al. Signature tau neuropathology in gray and white matter of corticobasal degeneration. Am J Pathol. 2002;

[183] Kouri N, Murray ME, Hassan A, et al. Neuropathological features of corticobasal degeneration presenting as corticobasal syndrome or Richardson syndrome. Brain. 2011;

[184] Ling H, Kovacs GG, Vonsattel JPG, et al. Astrogliopathy predominates the earliest stage of corticobasal degeneration pathology. Brain. 2016;

[185] Saeed U, Lang AE, Masellis M. Neuroimaging Advances in Parkinson's Disease and Atypical Parkinsonian Syndromes. Front. Neurol. 2020.

[186] Ishiki A, Harada R, Okamura N, et al. Tau imaging with [18F]THK-5351 in progressive supranuclear palsy. Eur J Neurol. 2017;

[187] Smith R, Schain M, Nilsson C, et al. Increased basal ganglia binding of 18F-AV-1451 in patients with progressive supranuclear palsy. Mov Disord. 2017;

[188] Cho H, Choi JY, Hwang MS, et al. Subcortical 18F-AV-1451 binding patterns in progressive supranuclear palsy. Mov Disord. 2017;

[189] Whitwell JL, Lowe VJ, Tosakulwong N, et al. [18F]AV-1451 tau positron emission tomography in progressive supranuclear palsy. Mov Disord. 2017;

[190] Passamonti L, Rodríguez PV, Hong YT, et al. 18F-AV-1451 positron emission tomography in Alzheimer's disease and progressive supranuclear palsy. Brain. 2017;

[191] Hammes J, Bischof GN, Giehl K, et al. Elevated in vivo [18F]-AV-1451 uptake in a patient with progressive supranuclear palsy. Mov. Disord. 2017.

[192] Coakeley S, Cho SS, Koshimori Y, et al. Positron emission tomography imaging of tau pathology in progressive supranuclear palsy. J Cereb Blood Flow Metab. 2017;

[193] Kepe V, Bordelon Y, Boxer A, et al. PET imaging of neuropathology in tauopathies: Progressive supranuclear palsy. J Alzheimer's Dis. 2013;

[194] Schonhaut DR, McMillan CT, Spina S, et al. 18F-flortaucipir tau positron emission tomography distinguishes established progressive supranuclear palsy from controls and Parkinson disease: A multicenter study. Ann Neurol. 2017;

[195] Brendel M, Schönecker S, Höglinger G, et al. [18F]-THK5351 PET correlates with topology and symptom severity in progressive supranuclear palsy. Front Aging Neurosci. 2018;

[196] Hsu JL, Chen SH, Hsiao IT, et al. 18F-THK5351 PET imaging in patients with progressive supranuclear palsy: associations with core domains and diagnostic certainty. Sci Rep. 2020; 
[197] Whitwell JL, Tosakulwong N, Botha H, et al. Brain volume and flortaucipir analysis of progressive supranuclear palsy clinical variants. NeuroImage Clin. 2020;

[198] Maruyama M, Shimada H, Suhara T, et al. Imaging of tau pathology in a tauopathy mouse model and in alzheimer patients compared to normal controls. Neuron. 2013;

[199] Kikuchi A, Okamura N, Hasegawa T, et al. In vivo visualization of tau deposits in corticobasal syndrome by 18 F-THK5351 PET. Neurology. 2016;

[200] McMillan CT, Irwin DJ, Nasrallah I, et al. Multimodal evaluation demonstrates in vivo 18FAV-1451 uptake in autopsy-confirmed corticobasal degeneration. Acta Neuropathol. 2016.

[201] Ali F, Whitwell JL, Martin PR, et al. [18F] AV-1451 uptake in corticobasal syndrome: the influence of beta-amyloid and clinical presentation. J Neurol. 2018;

[202] Tsai RM, Bejanin A, Lesman-Segev O, et al. 18F-flortaucipir (AV-1451) tau PET in frontotemporal dementia syndromes. Alzheimer's Res Ther. 2019;

[203] Lowe VJ, Curran G, Fang P, et al. An autoradiographic evaluation of AV-1451 Tau PET in dementia. Acta Neuropathol Commun. 2016;

[204] Brendel M, Barthel H, Van Eimeren T, et al. Assessment of 18F-PI-2620 as a Biomarker in Progressive Supranuclear Palsy. JAMA Neurol. 2020;

[205] Kroth H, Oden F, Molette J, et al. Discovery and preclinical characterization of [18F]PI2620, a next-generation tau PET tracer for the assessment of tau pathology in Alzheimer's disease and other tauopathies. Eur J Nucl Med Mol Imaging. 2019;

[206] Molinuevo JL, Ayton S, Batrla R, et al. Current state of Alzheimer's fluid biomarkers [Internet]. Acta Neuropathol. Springer Berlin Heidelberg; 2018. Available from: https://doi.org/10.1007/s00401-018-1932-X.

[207] Hampel H, Goetzl EJ, Kapogiannis D, et al. Biomarker-drug and liquid biopsy codevelopment for disease staging and targeted therapy: Cornerstones for Alzheimer's precision medicine and pharmacology. Front Pharmacol. 2019;10:1-10.

[208] Thijssen EH, Rabinovici GD. Rapid Progress Toward Reliable Blood Tests for Alzheimer Disease. JAMA Neurol. 2020;

[209] Janelidze S, Stomrud E, Smith R, et al. Cerebrospinal fluid p-tau217 performs better than ptau181 as a biomarker of Alzheimer's disease. Nat Commun [Internet]. 2020 [cited 2020 Dec 5];11. Available from: https://pubmed.ncbi.nlm.nih.gov/32246036/.

* A recent paper exploring the potential role of p-tau217 as a biomarker for AD

[210] Janelidze S, Berron D, Smith R, et al. Associations of Plasma Phospho-Tau217 Levels with Tau Positron Emission Tomography in Early Alzheimer Disease. JAMA Neurol. 2020;

* A recent paper exploring the potential role of p-tau217 as a biomarker for AD 
[211] Mollenhauer B, Locascio JJ, Schulz-Schaeffer W, et al. $\alpha$-Synuclein and tau concentrations in cerebrospinal fluid of patients presenting with parkinsonism: A cohort study. Lancet Neurol [Internet]. 2011 [cited 2020 Oct 10];10:230-240. Available from: https://pubmed.ncbi.nlm.nih.gov/21317042/.

[212] Hampel H, Lista S, Neri C, et al. Time for the systems-level integration of aging: Resilience enhancing strategies to prevent Alzheimer's disease. Prog Neurobiol [Internet]. 2019;181:101662. Available from: https://doi.org/10.1016/j.pneurobio.2019.101662.

[213] Toschi N, Lista S, Baldacci F, et al. Biomarker-guided clustering of Alzheimer's disease clinical syndromes. Neurobiol Aging. 2019;83:42-53.

[214] Hampel H, Caraci F, Cuello AC, et al. A Path Toward Precision Medicine for Neuroinflammatory Mechanisms in Alzheimer's Disease. Front Immunol. 2020;11. 
A
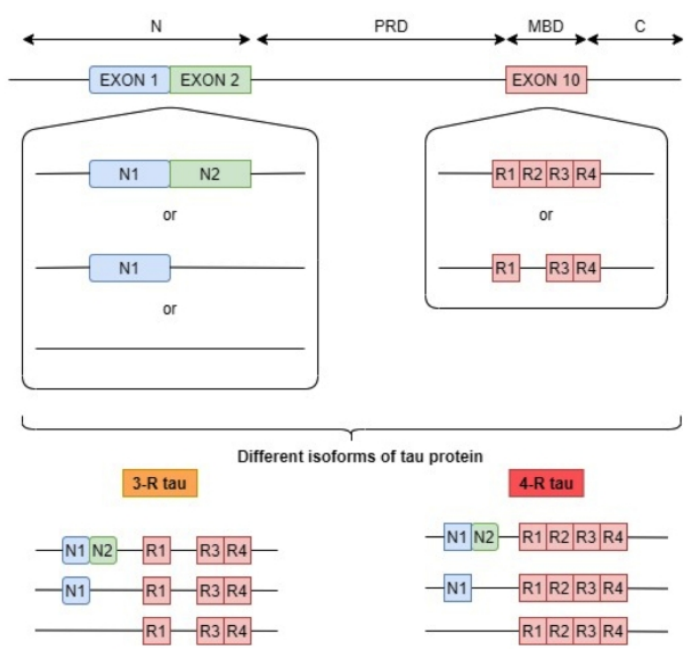

B
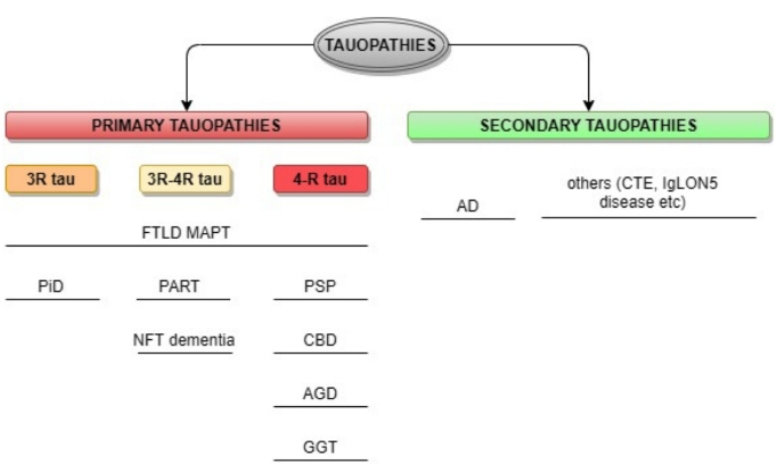

Figure 1. Tau isoforms and the pathological classification of tauopathies.Six tau isoforms are generated through the alternative splicing of exons 2,3 and 10 of the Microtubule-associated protein tau (MAPT) gene. The microtubule-binding domain (MBD) is encoded by exon 10, whose alternative splicing generates three tau isoforms with 3 or 4 repeats called $3 R$ and $4 R$ tau respectively (panel A). Tauopathies are classified accordingly into primary tauopathies, which are further divided into $3 R, 4 R$ and mixed $3 R / 4 R$ tauopathies, and secondary tauopathies (panel B).

$1057 \times 1730 \mathrm{~mm}(72 \times 72$ DPI $)$ 
Figure 2. Paper selection flow-chartThe paper selection algorithm for plasma tau in NDDs and CSF tau in NDDs other than AD is summarized in the figure. Abbreviations: AD: Alzheimer's Disease, CSF: cerebrospinal fluid, NDDs: neurodegenerative diseases.

$1057 \times 634 \mathrm{~mm}(72 \times 72 \mathrm{DPI})$ 


\begin{tabular}{|c|c|c|c|}
\hline Technology & Key characteristics & Tau species detected & Comments \\
\hline Simoa & $\begin{array}{l}\text { Fully-automated digitalized standard-ELISA. } \\
\text { How it works } \\
\text { 1. Specific antibody-coated paramagnetic } \\
\text { microbeads and fluorescence-emitting detection } \\
\text { antibodies are embedded with the analytes. } \\
\text { 2. The sample containing immunocomplexes } \\
\text { (bead, bound protein and detection antibody) is } \\
\text { loaded into arrays of subfemtomolar-sized } \\
\text { reaction chambers, large enough to hold one bead. } \\
\text { 3. The emitted signal reflects the presence of } \\
\text { single enzyme-associated immunocomplexes on } \\
\text { single beads. } \\
\text { (https://www.quanterix.com/simoa bead- } \\
\text { technology) }\end{array}$ & $\mathrm{N}$-terminal tau fragment & $\begin{array}{l}\text { Advantages } \\
\text { - greatest sensitivities; } \\
\text { - multiplexing (short turn-around times and } \\
\text { high throughput); } \\
\text { - flexibility and cost-saving; } \\
\text { - most established ultrasensitive technology } \\
\text { for blood-biomarkers in AD. } \\
\text { Limitations } \\
\text { - standardization of the protocol and further } \\
\text { cross-assay evaluations are needed. }\end{array}$ \\
\hline ECLIA & $\begin{array}{l}\text { Electrochemiluminescent labels generate light } \\
\text { when activated by the interaction with ligands. } \\
\text { The magnitude of the signal reflects the } \\
\text { concentration of the analyte. } \\
\text { How it works } \\
\text { 1. Labels interact with ligands; } \\
\text { 2. The interaction generates a luminescent signal, } \\
\text { whose magnitude is proportional to the analytes } \\
\text { concentration. } \\
\text { The reaction relies on a complex of ruthenium and } \\
\text { TPA and can be incorporated into different } \\
\text { immunoassays. (https://www.creative- } \\
\text { biolabs.com/drug-discovery/diagnostics/eclia- } \\
\text { based-kits-development.htm) }\end{array}$ & $\begin{array}{l}\text { t-tau } \\
\text { p-tau181 }\end{array}$ & $\begin{array}{l}\text { Advantages } \\
\text { - rapid measurement, wide measuring range, } \\
\text { high sensitivity (up to femtomolar range); } \\
\text { - low sample volume; } \\
\text { - MSD plattorm is a multiplex technology } \\
\text { enabling parallel measurements of different } \\
\text { biomarkers and matrices (e.g., plasma, CSF); } \\
\text { - optimal accuracy for different tau-species. } \\
\\
\text { Limitations } \\
\text { - further head-to-head comparisons are } \\
\text { required to assess sensitivity and specificity. }\end{array}$ \\
\hline
\end{tabular}


IP-LC/MS Combines immunoprecipitation and liquid chromatography (LC) /mass spectrometry (MS)

How it works

1. peptides are isolated with specific antibodies [30];

2. Peptides are physically separated based on their mass and biochemical properties by means of LC/MS.

An antibody-mediated reduction in the magnetic field generates a signal for analyte concentration quantification.

How it works

1. Magnetic antibody-coated nanoparticles oscillate under multiple AC magnetic fields; 2 . Binding of target molecules enlarges or clusters magnetic nanoparticles, leading to a reduction in their magnetic susceptibility, which is proportional to the concentration of the analytes.

\begin{tabular}{|l|l|}
\hline p-tau181 & $\begin{array}{l}\text { Limitations } \\
\text { - a multiple-step extraction phase to purify } \\
\text { and concentrate the analytes is required to } \\
\text { increase sensitivity and reduce the } \\
\text { interference of contaminants; } \\
\text { - labor-intensive, low-throughput and time- } \\
\text { consuming method. }\end{array}$ \\
\hline p-tau217 & $\begin{array}{l}\text { Advantages } \\
\text { - does not require beads to purify the antigens, } \\
\text { relying on a single-antibody detection strategy } \\
\text { - great sensitivity (up to the femtomolar } \\
\text { range) }\end{array}$ \\
\hline p-tau181 & $\begin{array}{l}\text { Limitations } \\
\text { - suboptimal specificity. }\end{array}$ \\
\\
\hline
\end{tabular}

Table 1. Overview of ultrasensitive measurement techniques for the detection of tau species in plasma

Abbreviations: AC: alternating current; AD: Alzheimer's disease; ECL: electrochemiluminescence; ECLIA: electrochemiluminescence immunoassay; ELISA: enzyme-linked immunosorbent assay; IMR: immunomagnetic reduction; IP/LC-MS: immunoprecipitation coupled with liquid chromatography-mass spectrometry; LoD: limit of detection; MSD: mesoscale discovery; p-tau181: phosphorylated-tau at threonine 181; p-tau217: phosphorylated-tau at threonine 217; Simoa: single molecule array; TPA: tripropylamine; t-tau: total tau. 


\begin{tabular}{|c|c|c|c|c|c|c|}
\hline Reference & Population & Study design & Technique & Tau species & Diagnostic value & Prognostic value \\
\hline $\begin{array}{l}\text { Lue LF. et al., } \\
2017\end{array}$ & $\begin{array}{l}n=129 \\
\text { - U.S. cohort: } n=32 \\
\text { CU }(n=16) ; A D \\
\text { dementia }(n=16) \\
\text { - Taiwan cohort: } n=97 \\
\text { CU }(n=66) ; A D \\
\text { dementia }(n=31)\end{array}$ & Cross-sectional & IMR & t-tau & $\begin{array}{l}\text { CU vs. probable AD: } \\
\text { - AuROC }=0.81 \text { (U.S. cohort) } \\
\text { - AuROC }=0.96 \text { (Taiwan cohort) } \\
\text { - AuROC }=0.92 \text { (combined cohort) } \\
\text { ( clinical diagnosis as reference) }\end{array}$ & NA \\
\hline $\begin{array}{l}\text { Mielke MM. et al., } \\
2017\end{array}$ & $\begin{array}{l}n=458 \\
\text { CU }(n=335) ; \text { MCI } \\
(n=123)\end{array}$ & b) $>0$ & $\begin{array}{l}\text { Simoa HD-1 } \\
\text { Analyzer }\end{array}$ & t-tau & NA & $\begin{array}{l}\text { Risk of evolving to } \mathrm{MCI} \text { in } \\
\text { CU: } \\
\text { - middle tertile }(\mathrm{HR}=2.43) \\
\text { - highest tertile }(\mathrm{HR}=2.02)\end{array}$ \\
\hline \multirow[t]{2}{*}{$\begin{array}{l}\text { Mielke MM et al., } \\
2018\end{array}$} & \multirow[t]{2}{*}{$\begin{array}{l}\mathrm{n}=269 \\
\mathrm{CU}(\mathrm{n}=172) ; \mathrm{MCI} \\
(\mathrm{n}=57) ; \mathrm{AD}(\mathrm{n}=40)\end{array}$} & \multirow[t]{2}{*}{ Cross-sectional } & $\begin{array}{l}\text { Simoa HD-1 } \\
\text { Analyzer }\end{array}$ & t-tau & $\begin{array}{l}\text { Amyloid-negative vs. amyloid- } \\
\text { positive: } \text { AuROC }=0.60 \\
\text { (amyloid-PET as reference) }\end{array}$ & NA \\
\hline & & & MSD & p-tau 181 & $\begin{array}{l}\text { Amyloid-negative vs. amyloid- } \\
\text { positive: } \text { AuROC }=0.80 \\
\text { (amyloid-PET as reference) }\end{array}$ & NA \\
\hline $\begin{array}{l}\text { Yang C. et al., } \\
2018\end{array}$ & $\begin{array}{l}\mathrm{n}=73 \\
\mathrm{CU}(\mathrm{n}=23) ; \mathrm{AD}-\mathrm{MCI} \\
(\mathrm{n}=29) ; \mathrm{AD}(\mathrm{n}=21)\end{array}$ & Cross-sectional & IMR & p-tau181 & $\begin{array}{l}\text { CU vs. AD-MCI: } A u R O C=0.85 \text {; } \\
\text { AD-MCI vs. AD: AuROC }=0.78 \\
\text { (clinical diagnosis as reference) }\end{array}$ & NA \\
\hline $\begin{array}{l}\text { Lin CH. et al., } \\
2018\end{array}$ & $\begin{array}{l}\mathrm{n}=205 \\
\text { CU }(\mathrm{n}=35) ; \text { PD } \\
(\mathrm{n}=102) ; \text { DLB }(\mathrm{n}=6) ; \\
\text { MSA }(\mathrm{n}=22) ; \text { PSP } \\
(\mathrm{n}=6) ; \text { CBD }(\mathrm{n}=3) ; \\
\text { FTD-P }(\mathrm{n}=6) ; \text { FTD } \\
\text { without P }(\mathrm{n}=25)\end{array}$ & Cross-sectional & IMR & p-tau181 & $\begin{array}{l}\text { FTD vs. parkinsonian syndromes } \\
\text { (PD, DLB, PSP and CBD) A } \beta_{1-42} \\
\text { x p-tau181 at a cut-off value of } \\
92.66(\mathrm{pg} / \mathrm{ml})^{2} \text { AuROC }=0.93 \\
\text { (clinical diagnosis as reference) }\end{array}$ & NA \\
\hline $\begin{array}{l}\text { Chen Z. et al., } \\
2019\end{array}$ & $\begin{array}{l}\mathrm{n}=151 \\
\text { - Discovery cohort: } \\
\mathrm{n}=65 \\
\mathrm{CU}(\mathrm{n}=19) ; \mathrm{AD}-\mathrm{MCI} \\
(\mathrm{n}=21) ; \mathrm{AD}(\mathrm{n}=25) \\
\text { - Validation cohort } \\
\mathrm{n}=86\end{array}$ & Cross-sectional & $\begin{array}{l}\text { Simoa HD-1 } \\
\text { Analyzer }\end{array}$ & $\begin{array}{l}\text { N-terminal } \\
\text { detected tau } \\
\text { (including tau } \\
\text { fragments) }\end{array}$ & $\begin{array}{l}\text { CU vs.AD-MCI: } A u R O C=0.88 \\
\text { CU vs AD: AuROC }=0.96 \\
\text { (clinical diagnosis as reference } \\
\text { standard) } \\
\text { (discovery cohort) }\end{array}$ & NA \\
\hline
\end{tabular}




\begin{tabular}{|c|c|c|c|c|c|c|}
\hline & $\begin{array}{l}\mathrm{CU}(\mathrm{n}=41) ; \mathrm{AD}-\mathrm{MCI} \\
(\mathrm{n}=22) ; \mathrm{AD}(\mathrm{n}=23)\end{array}$ & & & & & \\
\hline $\begin{array}{l}\text { Park JC. et al., } \\
2019\end{array}$ & $\begin{array}{l}n=76 \\
\text { CU }(n=52) ; \text { MCI }(n=9) ; \\
\text { AD dementia }(n=15)\end{array}$ & $\begin{array}{l}\text { Cross-sectional and } \\
\text { longitudinal }\end{array}$ & $\begin{array}{l}\text { Simoa HD-1 } \\
\text { Analyzer }(\mathrm{t}-\mathrm{tau}) \\
\mathrm{xMAP}\left(\mathrm{A} \beta_{1-42}\right)\end{array}$ & t-tau & $\begin{array}{l}\text { tau-positive vs. tau-negative: } \\
\text {-t-tau: } A \text { AuOC }=0.80 \\
\text {-t-tau/A } \beta_{1-42}: \text { AuROC }=0.89 \\
\text { (amyloid-PET as reference) }\end{array}$ & NA \\
\hline $\begin{array}{l}\text { Pase MP. et al., } \\
2019\end{array}$ & $\begin{array}{l}\mathrm{n}=1820 \\
\text { - Cohort } 1: \mathrm{n}=1453^{*} \\
(\mathrm{CU}>65 \text { years) } \\
\text { - Cohort } 2: \mathrm{n}=367^{\circ} \\
\text { (MCI, SMC) } \\
\text { *at FU: } \\
\text { - dementia onset } \mathrm{n}=134 \\
\text { AD dementia }(\mathrm{n}=105) \text {; } \\
\text { non-AD dementia } \\
(\mathrm{n}=29) \\
{ }^{\circ} \text { at FU: } \\
\text { - AD dementia onset } \\
(\mathrm{n}=19)\end{array}$ & Prospective & $\begin{array}{l}\text { Simoa } \\
\text { HD-1 Analyzer }\end{array}$ & t-tau & NA & $\begin{array}{l}\text { Cohort 1: } \\
\text { - each SD unit increase in } \\
\text { the log levels is associated } \\
\text { with a } 35 \% \text { increased risk } \\
\text { of } \mathrm{AD}(\mathrm{HR}=1.35) \\
\text { - after adjustment for } \\
\text { APOE } \varepsilon 4 \text { allele and } \\
\text { vascular risk factors: } \\
\text { - all dementia risk: } \\
\quad \mathrm{HR}=1.31 \\
\quad \mathrm{AD} \text { risk: HR=1.38 } \\
\text { Cohort } 2 \text { : } \\
\text { each } \mathrm{SD} \text { unit increase in } \\
\text { the log levels is associated } \\
\text { with a } 54 \% \text { increased risk } \\
\text { of AD (HR=2.33) after } \\
\text { adjusting for age and sex }\end{array}$ \\
\hline $\begin{array}{l}\text { Janelidze S. et al., } \\
2020\end{array}$ & $\begin{array}{l}\mathrm{n}=589 \\
- \text { Cohort } 1: \mathrm{n}=182 \\
\text { CU (n=64); MCI } \\
(\mathrm{n}=28) ; \mathrm{AD}(\mathrm{n}=38) ; \\
\text { non-AD dementia } \\
(\mathrm{n}=52) \\
\text { - Cohort } 2: \mathrm{n}=344 \\
\text { CU (n=219); MCI } \\
(\mathrm{n}=125) \\
- \text { Cohort } 3 \\
\text { (neuropathology } \\
\text { cohort): } n=63 \\
\text { AD }(n=16) ; \text { non-AD }\end{array}$ & $\begin{array}{l}\text { Both cross- } \\
\text { sectional and } \\
\text { longitudinal designs }\end{array}$ & MSD & p-tau 181 & $\begin{array}{l}\text { Cohort } 1 \text { : } \\
\text { - tau-positive vs. negative } \\
\text { participants AuROC }=0.87 \text { with } \\
\text { tau-PET as reference; } \\
\text { - AD vs. non-AD dementia: } \\
\text { AuROC }=0.94 \\
\text { (clinical diagnosis as reference) } \\
\text { Cohort } 1 \text { and cohort } 2 \text { combined: } \\
\text { - A } \beta-+ \text { vs. A } \beta \text { - participants } \\
\text { AuROC }=0.80 \\
\text { (A } \beta \text { PET as reference) }\end{array}$ & $\begin{array}{l}\text { Cohort 2: } \\
\text { higher p-tau levels are } \\
\text { associated with } \\
\text { progression to AD for both } \\
\mathrm{CU}(\mathrm{HR}=2.48) \text { and } \mathrm{MCI} \\
(\mathrm{HR}=3.07)\end{array}$ \\
\hline
\end{tabular}




\begin{tabular}{|c|c|c|c|c|c|c|}
\hline & dementia $(n=47)$ & & & & $\begin{array}{l}\text { Cohort 3: } \\
\text { AD vs. non-AD dementia: } \\
\text { AuROC }=0.85 \\
\text { (neuropathology as reference) }\end{array}$ & \\
\hline $\begin{array}{l}\text { Thijssen E. et al., } \\
2020\end{array}$ & $\begin{array}{l}\mathrm{n}=404 \\
\text { Cohort 1: } \mathrm{n}=362 \\
\text { CU }(\mathrm{n}=69) ; \text { MCI } \\
(\mathrm{n}=47) ; \text { AD }(\mathrm{n}=56) ; \\
\text { CBS }(\mathrm{n}=39) ; \text { PSP } \\
(\mathrm{n}=48) ; \text { bvFTD }(\mathrm{n}=50) ; \\
\text { nfvPPA }(\mathrm{n}=27) ; \text { svPPA } \\
(\mathrm{n}=26) \\
\text { Cohort } 2: \mathrm{n}=42(\mathrm{MCI}, \\
\text { AD) }\end{array}$ & $\begin{array}{l}\text { Cross-sectional } \\
\text { (retrospective) and } \\
\text { longitudinal }\end{array}$ & $\begin{array}{l}\text { MSD/Lilly } \\
\text { immunoassays }\end{array}$ & p-tau181 & $\begin{array}{l}\text { Cohort 1: } \\
\text { - AD vs. FTLD }(\mathrm{n}=190) \text { : } \\
\text { AuROC=0.89 } \\
\text { (clinical diagnosis as reference); } \\
\text { - A } \beta \text {-PET positive participants vs. } \\
\text { negative: AuROC = } 0.86 \text { with } \\
\text { amyloid-PET as reference; } \\
\text { - authopsy-confirmed AD }(\mathrm{n}=15) \\
\text { vs. FTLD-tau }(\mathrm{n}=52) \\
\text { AuROC=0.86 } \\
\text { (neuropathology as reference) }\end{array}$ & NA \\
\hline $\begin{array}{l}\text { Karikari T. et al., } \\
2020\end{array}$ & 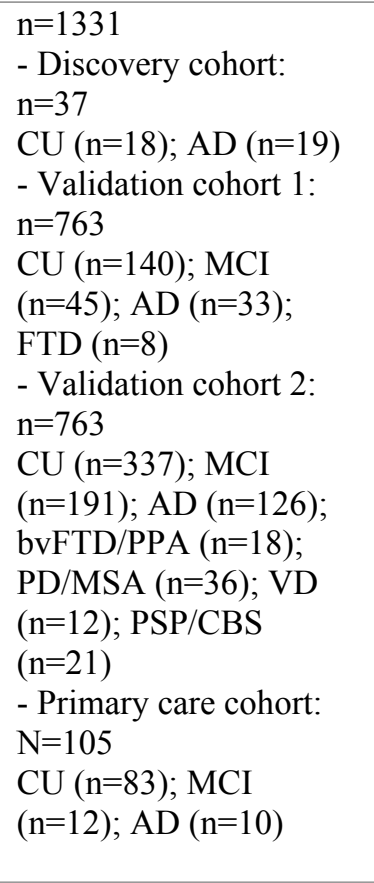 & Longitudinal & Simoa & p-tau181 & $\begin{array}{l}\text { Across cohorts } \\
\text { - AD vs. CU: AuROC }=0.90-0.98 \\
\text { Validation cohort } 1 \\
\text { - AD vs. FTD: AuROC }=0.76-0.82 \\
\text { (clinical diagnosis as reference) } \\
\text { - tau-PET + vs. tau-PET -: } \\
\text { AuROC }=0.83-0.93 \text { across cohorts } \\
\text { (tau-PET as reference) } \\
\text { - AD vs. amyloid- } \beta \text {-: } \\
\text { AuROC }=0.99 \\
\text { (amyloid-PET as reference) } \\
\text { Validation cohort } 2 \\
\text { - AD vs VaD: AuROC }=0.92 \\
\text { - AD vs PSP/CBS: AuROC }=0.89 \\
\text { - AD vs PD/MSA: AuROC }=0.82 \\
\text { (clinical diagnosis as reference) } \\
\text { Primary care cohort } \\
\text { - AD vs CU young adults: } \\
\text { AuROC }=1.0 \\
\text { - AD vs CU older adults: } \\
\text { AuROC }=0.84 \\
\text { (clinical diagnosis as reference) }\end{array}$ & NA \\
\hline $\begin{array}{l}\text { Palmqvist S. et al., } \\
2020\end{array}$ & $\begin{array}{l}\mathrm{n}=1402 \\
- \text { Cohort } 1 \\
\text { (neuropathology } \\
\text { cohort): } \mathrm{n}=81\end{array}$ & Cross-sectional & $\begin{array}{l}\text { MSD/Lilly } \\
\text { immunoassays }\end{array}$ & p-tau 217 & $\begin{array}{l}\text { Cohort 1: } \\
\text { AD vs. non-AD: AuROC }=0.89 \\
\text { (neuropathology as reference) } \\
\text { Cohort 2: }\end{array}$ & NA \\
\hline
\end{tabular}




\begin{tabular}{|c|c|c|c|c|c|c|}
\hline & $\begin{array}{l}\text { AD }(n=34) ; \text { non-AD } \\
(n=47) \\
\text { - Cohort 2: } n=699 \\
\text { CU (n=301); MCI } \\
(n=178) ; \text { AD dementia } \\
(n=121) ; \text { non-AD } \\
\text { dementia: } \\
\text { PD/PDD/MSA, } \\
\text { PSP/CBS, bvFTD/PPA, } \\
\text { VD ( } n=99) \\
\text { - Cohort 3: } n=622 \\
\text { PSEN1 mutation } \\
\text { carriers n=365; age- and } \\
\text { sex-matched } \\
\text { noncarriers: } n=257\end{array}$ & & & & $\begin{array}{l}\text { - AD vs. non-AD dementia: } \\
\text { AuROC }=0.96 \text { (clinical diagnosis } \\
\text { as reference) } \\
\text { - tau + vs.tau -: AuROC }=0.93 \\
\text { (tau-PET as reference) } \\
\text { - amyloid + vs. amyloid -: } \\
\text { AuROC }=0.87 \\
\text { (amyloid-PET as reference) }\end{array}$ & \\
\hline \multirow[t]{2}{*}{$\begin{array}{l}\text { Barthélemy NR. et } \\
\text { al., } 2020\end{array}$} & \multirow{2}{*}{$\begin{array}{l}\mathrm{n}=128 \\
\text { - Discovery cohort: } \\
\mathrm{n}=36 \\
\mathrm{CU}(\mathrm{n}=17) \text {; non-AD } \\
\mathrm{MCI}(\mathrm{n}=2) ; \text { preclinical } \\
\text { AD }(\mathrm{n}=5) ; \text { AD-MCI } \\
(\mathrm{n}=8) ; \text { moderate AD } \\
(\mathrm{n}=2) \\
\text { - Validation cohort: } \\
\mathrm{n}=92 \\
\mathrm{CU}(\mathrm{n}=31) ; \text { non-AD } \\
\mathrm{MCI}(\mathrm{n}=11) ; \text { preclinical } \\
\text { AD }(\mathrm{n}=20) ; \text { AD-MCI } \\
(\mathrm{n}=24) ; \text { moderate AD } \\
(\mathrm{n}=6)\end{array}$} & \multirow[t]{2}{*}{ Cross-sectional } & \multirow[t]{2}{*}{ IP-LC/MS } & p-tau181 & $\begin{array}{l}\text { Amyloid + vs. - } \\
\text { - discovery cohort: AuROC }=0.98 \\
\text { - validation cohort: } A u R O C=0.75 \\
\text { (amyloid-PET or } C S F A \beta_{1-42} / A \beta_{1-} \\
40 \text { as reference) }\end{array}$ & NA \\
\hline & & & & p-tau217 & $\begin{array}{l}\text { Amyloid positive vs. amyloid- } \\
\text { negative subjects: } \\
\text { - discovery cohort: } A u R O C=0.99 \\
\text { - validation cohort: } A u R O C=0.92 \\
\text { ( amyloid-PET or CSF } A \beta_{1-} \\
{ }_{42} / A \beta_{1-40} \text { as reference) }\end{array}$ & NA \\
\hline $\begin{array}{l}\text { Janelidze S. et al., } \\
2020\end{array}$ & $\begin{array}{l}n=490 \\
\text { CU }(n=225) ; \text { SCI } \\
(n=89) ; \operatorname{MCI}(n=176)\end{array}$ & $\begin{array}{l}\text { Cross-sectional and } \\
\text { longitudinal }\end{array}$ & $\begin{array}{l}\text { MSD/Lilly } \\
\text { immunoassays }\end{array}$ & p-tau 217 & $\begin{array}{l}\mathrm{A} \beta-\mathrm{PET}^{+} / \text {tau-PET- }^{-} \mathrm{CU} \text { vs. A } \beta- \\
\mathrm{PET}^{-} / \text {tau-PET}^{-}: \text {AuROC }=0.832 \\
\text { (amyloid-PET as reference ) }\end{array}$ & NA \\
\hline
\end{tabular}


Table 2. Diagnostic and prognostic value of plasma tau species in NDDs assessed with ultrasensitive measurement techniques

Abbreviations: AD: Alzheimer's Disease; AuROC: area under the receiver operating curve; A $\beta$ : amyloid $\beta$; A $\beta 1-40$ : amyloid $\beta$-peptide 1-40; A $\beta 1-42$ : amyloid $\beta$-peptide 1-42; bvFTD: behavioral variant Frontotemporal Dementia; CBS: Corticobasal Syndrome; CU: Cognitively Unimpaired; FTD: Frontotemporal Dementia; FTLD: Frontotemporal Lobar Degeneration; FTD-P: Frontotemporal Dementia with parkinsonism; FU: follow-up; HR: hazard ratio; IMR: immunomagnetic reduction; IP LC/MS: immunoprecipitation coupled to liquid chromatography/mass spectrometry; MCI: mild cognitive impairment; MSD: meso scale discovery; MSA: Multiple System Atrophy; NA: not assessed; nfvPPA: non-fluent variant primary progressive aphasia; PD: Parkinson's disease; PDD: Parkinson's disease dementia; PPA: Primary Progressive Aphasia; PSP: Progressive Supranuclear Palsy; p-tau181: phospho-tau181; p-tau217: phosphotau217; SD: standard deviation; Simoa: single molecule array; SCI: subjective cognitive impairment;_-SMC: subjective memory complainer; svPPA: semantic variant primary progressive aphasia; t-tau: total-tau; VaD: vascular dementia; xMAP: multi-analyte profiling. 


\begin{tabular}{|c|c|c|c|c|c|c|}
\hline Reference & Population & Study design & Technique & Tau species & Diagnostic value & Prognostic value \\
\hline $\begin{array}{l}\text { Magdalinou NK. et } \\
\text { al., } 2015\end{array}$ & $\begin{array}{l}\mathrm{n}=190 \\
\text { CU }(\mathrm{n}=30) ; \text { PD }(\mathrm{n}=31) ; \\
\text { PSP }(\mathrm{n}=33) ; \text { CBS } \\
(\mathrm{n}=14) ; \text { MSA }(\mathrm{n}=31) ; \\
\text { AD }(\mathrm{n}=26) ; \text { FTD } \\
(\mathrm{n}=16) ; \text { "unclassified" } \\
\text { parkinsonism }(\mathrm{n}=9)\end{array}$ & Prospective & $\begin{array}{l}\text { ELISA } \\
\text { INNOTEST }(\mathrm{t}- \\
\left.\text { tau; } \mathrm{p}-\mathrm{tau} ; \mathrm{A} \beta_{1-42}\right)\end{array}$ & t-tau, p-tau & $\begin{array}{l}\text { t-tau, p-tau combined with } A \beta_{1-42} \text {, } \\
\text { NFL, } \alpha \text {-syn, sAPP } \alpha \text {, sAPP } \beta, \text { MCP- } \\
\text { 1, YKL-40): } \\
\text { - PD vs. APSs (PSP, CBS, MSA): } \\
\text { AuROC }=0.95 \\
\text { - PD vs. PSP: AuROC }=0.95 \\
\text { - PD vs. CBS: AuROC }=0.98 \\
\text { - CBS vs. AD/FTD: AuROC }=0.93 \\
\text { (clinical diagnosis as reference) }\end{array}$ & NA \\
\hline $\begin{array}{l}\text { Wagshal D. et al., } \\
2015\end{array}$ & $\begin{array}{l}n=87 \\
\operatorname{CU}(n=26) ; \operatorname{AD}(n=37) \\
\operatorname{PSP}(n=24)\end{array}$ & Cross-sectional & $\begin{array}{l}\text { INNOBHA } \\
\text { AlzBie3 with } \\
\text { xMAP platform } \\
\text { (t-tau; p-tau181)/ } \\
\text { ELISA_s } \\
\text { (alternative N- } \\
\text { terminal and mid- } \\
\text { tau fragments) }\end{array}$ & $\begin{array}{l}\text { t-tau, } p \text {-tau } 181 \\
\text { tau fragments }\end{array}$ & $\begin{array}{l}\text { t-tau, p-tau181 and tau fragments: } \\
\text { - AD vs. PSP: AuROC }=0.79-0.95 \text {; } \\
\text { - AD vs. CU: AuROC }=0.65-0.73 \\
\text { N-terminal fragment tau12-BT2 } \\
\text { shows the best accuracy: } \\
\text { - AD vs PSP: AuROC }=0.95 \\
\text { - AD vs CU: AuROC }=0.73\end{array}$ & NA \\
\hline $\begin{array}{l}\text { Wilke C. et al., } \\
2015\end{array}$ & $\begin{array}{l}n=180 \\
C U(n=20) ; \operatorname{ALS}(n=60)\end{array}$ & Cross-sectional & $\begin{array}{l}\text { ELISA } \\
\text { INNOTEST }\end{array}$ & t-tau, p-tau181 & $\begin{array}{l}\text { CU vs. ALS: } \\
\text { - t-tau: AuROC }=0.68 \\
\text { - p-tau/t-tau: AuROC }=0.75 \\
\text { (clinical diagnosis as reference) }\end{array}$ & NA \\
\hline $\begin{array}{l}\text { Llorens F. et al., } \\
2016\end{array}$ & $\begin{array}{l}n=140 \\
\text { CU }(n=55) ; \text { ET }(n=8) ; \\
\text { PD }(n=40) ; \text { PDD } \\
(n=10) ; \text { DLB }(n=18) ; \\
\text { MSA }(n=11)\end{array}$ & Cross-sectional & $\begin{array}{l}\text { ELISA (t- } \\
\text { tau)/MSD ( } \alpha-\text {-syn })\end{array}$ & t-tau & $\begin{array}{l}\text { CU vs. DLB: } \\
\text { - t-tau/ } \alpha \text {-syn: AuROC }=0.88 \\
\text { - t-tau: AuROC }=0.77 \\
\text { (clinical diagnosis as reference) }\end{array}$ & NA \\
\hline $\begin{array}{l}\text { Bourbouli M. et } \\
\text { al., } 2017\end{array}$ & $\begin{array}{l}\mathrm{n}=100 \\
\text { CU }(\mathrm{n}=17) ; \text { ALS } \\
(\mathrm{n}=32) ; \text { FTD }(\mathrm{n}=51)\end{array}$ & Cross-sectional & Sandwich-ELISA & t-tau, p-tau 181 & $\begin{array}{l}\text { t-tau: } \\
\text { - CU vs. ALS: AuROC }=0.80 \\
\text { - CU vs. FTD: AuROC=0.77; } \\
\text { TDP-43 x t-tau/p-tau181 in: } \\
\text { - CU vs. ALS: AuROC }=0.97 \\
\text { - CU vs. FTD: AuROC }=0.90 \\
\text { (clinical diagnosis as reference) }\end{array}$ & NA \\
\hline $\begin{array}{l}\text { Constantinides VC. } \\
\text { et al., } 2017\end{array}$ & $\begin{array}{l}\mathrm{n}=86 \\
\text { CU }(\mathrm{n}=18) ; \text { PSP } \\
(\mathrm{n}=19) ; \text { MSA }(\mathrm{n}=15) ; \\
\text { CBD }(\mathrm{n}=17) ; \mathrm{PD} \\
(\mathrm{n}=17)\end{array}$ & Cross-sectional & $\begin{array}{l}\text { ELISA } \\
\text { INNOTEST }\end{array}$ & t-tau & $\begin{array}{l}\text { t-tau/A } \beta_{1-42} \text { in: } \\
\text { - MSA vs. PD: AuROC }=0.80 \\
\text { - cut-off point } 0.344: 71.4 \% \\
\text { sensitivity, } 93.3 \% \text { specificity } \\
\text { - cut-off point } 0.305: 78.6 \% \\
\text { sensitivity, } 80 \% \text { sensitivity } \\
\text { (clinical diagnosis as reference) }\end{array}$ & NA \\
\hline
\end{tabular}




\begin{tabular}{|c|c|c|c|c|c|c|}
\hline $\begin{array}{l}\text { Delgado-Alvarado } \\
\text { M. et al., } 2017\end{array}$ & $\begin{array}{l}\mathrm{n}=80 \\
\mathrm{CU}(\mathrm{n}=40) ; \mathrm{PD}(\mathrm{n}=40)\end{array}$ & Cross-sectional & $\begin{array}{l}\text { ELISA } \\
\text { INNOTEST }\end{array}$ & t-tau, p-tau 181 & $\begin{array}{l}\text { CU vs. PD: } \\
\text { - t-tau/ } \alpha \text {-syn: AuROC }=0.79 \\
\text { - p-tau181/ } \alpha \text {-syn: AuROC }=0.86 \\
\text { - p-tau } 181 / \alpha \text {-syn and TNF- } \alpha \text { : } \\
\text { AuROC }=0.91 \\
\text { (clinical diagnosis as reference) }\end{array}$ & NA \\
\hline $\begin{array}{l}\text { Irwin DJ. et al., } \\
2018\end{array}$ & $\begin{array}{l}\mathrm{n}=83 \\
\mathrm{CU}(\mathrm{n}=36) ; \text { autopsy- } \\
\text { confirmed } \mathrm{AD}(\mathrm{n}=23) ; \\
\text { autopsy-confirmed DLB } \\
(\mathrm{n}=24)\end{array}$ & Cross-sectional & xMAP platform & t-tau, p-tau 181 & $\begin{array}{l}\text { Predicting } \alpha \text {-syn }+ \text { AD pathology: } \\
\text { - t-tau: AuROC }=0.80 \\
\text { - p-tau } / \mathrm{A} \beta_{1-42}: \text { AuROC }=0.80 \\
\text { - t-tau } / \mathrm{A} \beta_{1-42}: \text { AuROC }=0.92 ; \\
\text { Predicting of neocortical Lewy } \\
\text { Bodies pathology t-tau } / \mathrm{A} \beta_{1-42}: \\
\text { AuROC }=0.76 \\
\text { (neuropathology as reference) }\end{array}$ & NA \\
\hline $\begin{array}{l}\text { Scarafino A. et al., } \\
2018\end{array}$ & $\begin{array}{l}\mathrm{n}=166 \\
\text { ALS (n=85); AM } \\
(\mathrm{n}=30) ; \text { non-NDDs } \\
(\mathrm{n}=51)\end{array}$ & Longitudinal & ELISA & t-tau, p-tau 181 & $\begin{array}{l}\text { t-tau in: } \\
\text { - ALS vs. AM: AuROC }=0.70 \\
\text { - ALS vs. non-NDDs: } \\
\text { AuROC }=0.74 \\
\text { (clinical diagnosis as reference) }\end{array}$ & $\begin{array}{l}\text { Higher baseline } t \text {-tau } \\
\text { levels are associated with } \\
\text { decreased overall } \\
\text { survival }(H R=2.08)\end{array}$ \\
\hline $\begin{array}{l}\text { Schirinzi T. et al., } \\
2018\end{array}$ & $\begin{array}{l}\mathrm{n}=128 \\
\mathrm{CU}(\mathrm{n}=58) ; \mathrm{PSP} \\
(\mathrm{n}=39) ; \mathrm{PD}(\mathrm{n}=31)\end{array}$ & Cross-sectional & $\begin{array}{l}\text { ELISA } \\
\text { INNOTEST }\end{array}$ & t-tau, p-tau 181 & $\begin{array}{l}\text { PSP vs. CU: } \\
\text { - p-tau (cut-off } 206 \mathrm{pg} / \mathrm{ml}) \text { : } \\
\text { AuROC }=0.67 \\
\text { - t-tau (cut-off } 34.5 \mathrm{pg} / \mathrm{ml} \text { ): } \\
\text { AuROC }=0.69 ; \\
\text { PSP vs. PD: } \\
\text { - p-tau/t-tau (cut-off } 0.185 \mathrm{pg} / \mathrm{ml} \text { ): } \\
\text { AuROC=0.70 } \\
\text { (clinical diagnosis as reference) }\end{array}$ & NA \\
\hline $\begin{array}{l}\text { Constantinescu R. } \\
\text { et al., } 2019\end{array}$ & $\begin{array}{l}\mathrm{n}=151 \\
\text { PD }(\mathrm{n}=68) ; \operatorname{APS}(\mathrm{n}=83) \\
\text { including MSA }(\mathrm{n}=34), \\
\text { PSP }(\mathrm{n}=34), \text { CBS } \\
(\mathrm{n}=15)\end{array}$ & Prospective & $\begin{array}{l}\text { ELISA } \\
\text { INNOTEST }\end{array}$ & t-tau, p-tau181 & NA & $\begin{array}{l}\text { In PSP t-tau is associated } \\
\text { with increased mortality } \\
(\mathrm{HR}=9.59)\end{array}$ \\
\hline $\begin{array}{l}\text { Abu-Rumeileh S. } \\
\text { et al., } 2020\end{array}$ & $\begin{array}{l}\mathrm{n}=169 \\
\mathrm{CU}(\mathrm{n}=43) ; \mathrm{ALS} \\
(\mathrm{n}=80) ; \mathrm{AM}(\mathrm{n}=46)\end{array}$ & Longitudinal & $\begin{array}{l}\text { ELISA } \\
\text { INNOTEST }\end{array}$ & t-tau, p-tau 181 & $\begin{array}{l}\text { ALS vs. CU: } \\
\text { - t-tau: AuROC }=0.74 \\
\text { - p-tau/t-tau:AuROC }=0.81 \\
\text { ALS vs. AM: } \\
\text { - p-tau/t-tau: } \text { AuROC }=0.75 \\
\text { (clinical diagnosis as reference) }\end{array}$ & NA \\
\hline $\begin{array}{l}\text { Bousiges O. et al., } \\
2020\end{array}$ & $\begin{array}{l}\mathrm{n}=166 \\
\mathrm{CU}(\mathrm{n}=21) ; \mathrm{DLB}\end{array}$ & Cross-sectional & $\begin{array}{l}\text { ELISA } \\
\text { INNOTEST }\end{array}$ & t-tau; p-tau 181 & $\begin{array}{l}\text { t-tau: } \\
\text { - DLB vs. AD: AuROC=0.92 }\end{array}$ & NA \\
\hline
\end{tabular}




\begin{tabular}{|c|c|c|c|c|c|c|}
\hline & $(\mathrm{n}=67) ; \mathrm{AD}(\mathrm{n}=65)$ & & & & $\begin{array}{l}\text { - DLB vs. AD: AuROC }=0.94 ; \\
\text { p-tau181: } \\
\text { - DLB vs. AD: AuROC }=0.93 \\
\text { (clinical diagnosis as reference) }\end{array}$ & \\
\hline Ye LQ. et al., 2020 & $\begin{array}{l}\mathrm{n}=240 \\
\text { CU }(n=24) ; \operatorname{AD}(n=82) ; \\
\text { FTD }(n=20) ; \text { HD } \\
(n=27) ; \text { MSA }(n=24) ; \\
\text { SCA3 }(n=27) ; \text { ALS } \\
(n=36)\end{array}$ & Cross-sectional & ELISA & t-tau; p-tau 181 & $\begin{array}{l}\text { t-tau: } \\
\text { - CU vs. FTD: AuROC }=0.76 \\
\text { - ALS vs. AD AuROC }=0.87 \\
\text { t-tau/A } \beta_{1-42}: \\
\text { - CU vs. FTD: AuROC }=0.80 \\
\text { - AD vs. FTD: AuROC }=0.95 \\
\text { (clinical diagnosis as reference) }\end{array}$ & NA \\
\hline
\end{tabular}

Table 3. Diagnostic and prognostic value of CSF tau species in non-AD NDDs

Abbreviations: $\alpha$-syn: $\alpha$-synuclein; AD: Alzheimer's Disease; ALS: Amyotrophic Lateral Sclerosis; AM: Amyotrophic Lateral Sclerosis mimics; APS: Atypical Parkinsonian Syndrome; AuROC: area under the receiver operating curve; A $\beta_{1-42}$ : amyloid $\beta$-peptide $1-42$; CBS: Corticobasal Syndrome; CU: Cognitively Unimpaired; DLB: Dementia with Lewy Bodies; ELISA: enzyme-linked immunosorbent assay; ET: Essential Tremor; FTD: Frontotemporal Dementia; HD: Huntington's Disease; HR: hazard ratio; MCP-1: monocyte chemoattractant protein-1; MSD: mesoscale discovery; MSA: multiple system atrophy; NA: not assessed; NDD: neurodegenerative disease; NFL: neurofilament light chain; PD: Parkinson's Disease; PDD: Parkinson's Disease Dementia; PSP: Progressive Supranuclear Palsy; p-tau: phopsho-tau; p-tau181: phospho-tau181; sAPP $\alpha$ : soluble amyloid precursor protein $\alpha$; sAPP $\beta$ : soluble amyloid precursor protein $\beta$; SCA3: spinocerebellar ataxia-type 3; TDP-43: TAR DNA-binding protein 43; t-tau: total-tau; TNF- $\alpha$ : tumor necrosis factor $\alpha$; xMAP: multi-analyte profiling; YKL-40: chitinase-3-like protein 1. 